\title{
Das Gitterpunktproblem in der hyperbolischen Ebene
}

\author{
Dissertation \\ zur Erlangung des Doktorgrades \\ der Mathematisch-Naturwissenschaftlichen Fakultäten \\ der Georg-August-Universität zu Göttingen
}

vorgelegt von Jan Thirase

aus Lüneburg

Göttingen 2000 
D 7

Referent: Prof. Patterson

Korreferent: Prof. Burmann

Tag der mündlichen Prüfung: 1.11.2000 


\section{Inhaltsverzeichnis}

1. Einleitung 4

1.1. Grundlegende Begriffe und Notationen . . . . . . . . . . . . . . 5

1.2. Zur Geschichte des Gitterpunktproblems . . . . . . . . . . . . . . . 7

1.3. Ergebnisse . . . . . . . . . . . . . . . . . . . . 10

1.4. Die verwendete Methode . . . . . . . . . . . . . . . . . . . 15

2. Das klassische Kreisproblem 19

2.1. Die Spektraltheorie euklidischer Gitter . . . . . . . . . . . . . . . . . 19

2.2. Abschätzungen der Gitterpunktzählfunktion. . . . . . . . . . . . 22

3. Das Gitterpunktproblem in der hyperbolischen Ebene 29

3.1. Die Spektraltheorie Fuchsscher Gruppen . . . . . . . . . . . . . . . . . . 29

3.1.1. Die Spektralzerlegung von Punktpaarinvarianten . . . . . . . . . 30

3.1.2. Das $\mathbf{L}^{2}$-Spektrum Fuchsscher Gruppen . . . . . . . . . . . . . 34

3.2. Die Asymptotik der Gitterpunktzählfunktion . . . . . . . . . . . . . 35

3.3. Numerische Abschätzungen der Zählfunktion . . . . . . . . . . . . . 42

3.4. Anwendungen . . . . . . . . . . . . . . . . . 53

3.4.1. Ein Test auf die Existenz eines zweiten kleinen Eigenwertes . . . . 53

3.4.2. Approximation des Konvergenzexponenten und der ersten Eigenfunktion . . . . . . . . . . . . . . . . 55

A. Die Legendrefunktionen $\quad \mathbf{5 9}$

A.1. Abschätzungen für kleine Eigenwerte . . . . . . . . . . . . . . . . . 60

A.2. Abschätzungen für große Eigenwerte . . . . . . . . . . . . . 63

$\begin{array}{ll}\text { B. Einige Abschätzungen } & 65\end{array}$

$\begin{array}{ll}\text { C. Das Computerprogramm } & 69\end{array}$ 


\section{Einleitung}

Carl Friedrich Gauß formulierte Anfang des neunzehnten Jahrhunderts die Fragestellung, die heute als das klassische Kreisproblem bezeichnet wird. Er stellte fest, daß die Anzahl von Gitterpunkten aus $\mathbb{Z}^{2}$ in einem Kreis mit Radius $R$ etwa gleich dem Flächeninhalt dieses Kreises ist, und schätzte den bei dieser Näherung gemachten Fehler über den Umfang des Kreises ab. Gauß fragte nun, inwieweit sich diese Abschätzung verbessern läßt. Fortschritte in dieser Frage wurden erst Anfang des zwanzigsten Jahrhunderts erzielt, und bis heute ist keine abschließende Antwort gefunden worden.

In dieser Arbeit werden wir neben dem klassischen Kreisproblem vor allem dessen Verallgemeinerung auf die diskreten Bewegungsgruppen in der hyperbolischen Ebene auf die sogenannten Fuchsschen Gruppen betrachten. Es waren Mathematiker wie Felix Klein und Henri Poincaré, die Ende des neunzehnten Jahrhunderts die Fuchsschen Gruppen als den richtigen Zugang zu der von Riemann revolutionierten Funktionentheorie erkannten und in das Zentrum ihrer mathematischen Forschung stellten. Bis heute sind die Fuchsschen Gruppen und ihre Verallgemeinerung auf höherdimensionale Räume -die Kleinschen Gruppen- Gegenstand intensiver Forschung. Wie groß ihre Bedeutung für zahlreiche Teilgebiete aus der Mathematik ist, tritt dabei immer stärker hervor. Methoden aus dem Gebiet der Funktionentheorie, der Geometrie, der dynamischen Systeme sowie der fraktalen Geometrie und der Streu- und Spektraltheorie finden hier Anwendung und können in ihrer Wechselwirkung untersucht werden.

Die Gitterpunktzählfunktion einer Fuchsschen Gruppe zählt die Bilder eines festen Punktes unter der Operation der Gruppe in einem vorgegebenen Kreis. Das Studium dieser Zählfunktion bietet einen wesentlich tieferen Einblick in das Wesen von Gitterpunktproblemen als es die Betrachtung des klassischen Kreisproblems vermag. Erscheint die Anwendung von Spektraltheorie auf das Gitterpunktproblem im euklidischen Fall vielleicht noch als bloße Methode, so zeigt sich beim Studium der Gitterpunktzählfunktionen Fuchsscher Gruppen, wie eng das Gitterpunktproblem tatsächlich mit der Spektraltheorie zusammenhängt. Das Verhalten der Gitterpunktzählfunktion einer Fuchsschen Gruppe $G$ wird durch die diskreten Eigenwerte des Laplace-Beltrami Operators auf der zu $G$ gehörenden Riemannschen Fläche im Intervall $[0,1 / 4)$ und die zugehörigen Eigenfunktionen bestimmt.

In Kapitel 2 wird das klassische Kreisproblem behandelt und in Kapitel 3 das Gitterpunktproblem geometrisch endlicher Fuchsscher Gruppen. Es geht insbesondere darum, nicht nur die Ordnung der Restterme in den asymptotischen Formeln abzuschätzen, sondern auch obere und untere Schranken der Zählfunktionen mit allen enthaltenen Konstanten anzugeben. Dazu wird Hubers Methode aus [15] verallgemeinert, was durch 
eine Anwendung der Spektraltheorie von Maurin [21] gelingt, die schon von S. J. Patterson in [29] auf Fuchssche Gruppen angewendet wurde. Im Falle geometrisch endlicher Fuchsscher Gruppen mit einem Fundamentalbereich von unendlichem Flächeninhalt ermöglichen es die gewonnenen Ergebnisse, den Konvergenzexponenten bzw. die Hausdorffdimension der Limesmenge durch das Auszählen von Gitterpunkten mit beliebig vorgegebener Genauigkeit zu approximieren. Zur Demonstration dieses Ergebnisses wird mit einem Computerprogramm die Zählfunktion einer speziellen Heckegruppe bestimmt und damit eine Abschätzung ihres Konvergenzexponenten gegeben. Außerdem kann für cofinite Fuchssche Gruppen ein Test auf die Existenz eines (neben dem Eigenwert 0) weiteren diskreten Eigenwertes angegeben werden.

An dieser Stelle möchte ich mich bei Herrn Prof. Patterson für die Vergabe des Themas und seine Unterstützung während der Erstellung dieser Arbeit bedanken. Mein weiterer Dank gilt der Graduiertenförderung des Landes Niedersachsen sowie der Deutschen Forschungsgemeinschaft für die finanzielle Unterstützung.

\subsection{Grundlegende Begriffe und Notationen}

In diesem Abschnitt sollen kurz die für das Verständnis dieser Arbeit grundlegenden Begriffe in Erinnerung gerufen werden. Außerdem werden einige Notationen erklärt.

Wir erklären zuerst Gitter in der euklidischen Ebene. Es seien $v_{1}, v_{2}$ zwei linear unabhängige Vektoren des $\mathbb{R}^{2}$. Sie definieren eine Matrix $A:=\left(v_{1}^{t}, v_{2}^{t}\right)$. Diese definiert ein Gitter $Z \subset \mathbb{R}^{2}$ mit den Punkten

$$
\omega=A^{t} k \quad k \in \mathbb{Z}^{2} .
$$

Für das Gitter $Z$ definieren wir nun die Gitterpunktzählfunktion als

$$
N(R, p):=\#\{\omega \in Z:\|\omega-p\| \leq R\},
$$

wobei $\|\cdot\|$ die euklidische Norm ist. Den Ausdruck

$$
d(R, p):=N(R, p)-\frac{\pi}{\operatorname{area}(T)} R^{2}
$$

mit $T:=\mathbb{R}^{2} / Z$ werden wir als Restterm bezeichnen. Hierbei bezeichnet area $(T)$ den Flächeninhalt von $T$.

Das zentrale Thema dieser Arbeit ist die Gitterpunktzählfunktion Fuchsscher Gruppen. Um Fuchssche Gruppen zu erklären, benötigen wir den Begriff der hyperbolischen Ebene. Als Modell für die hyperbolische Ebene verwenden wir die Einheitskreisscheibe

$$
D:=\{z \in \mathbb{C}:|z|<1\}
$$

mit der Metrik

$$
d s^{2}=\left(1-|z|^{2}\right)^{-2}|d z|^{2},
$$

die eine Abstandsfunktion $d(\cdot, \cdot)$ auf $D$ gibt. Die Gruppe der $d$-invarianten Automorphismen von $D$ sei mit Con (1) bezeichnet. Eine diskrete Untergruppe von Con (1) wird 
als Fuchssche Gruppe bezeichnet. Sie operiert diskontinuierlich auf $D$. Ein anderes zu $D$ konform-äquivalentes Modell des hyperbolischen Raumes hat als Grund-Raum die obere Halbebene

$$
\mathbb{H}:=\{z \in \mathbb{C}: \operatorname{Im}(z)>0\}
$$

Die Abbildung

$$
\begin{aligned}
f: \mathbb{H} & \rightarrow(D, d) \\
z & \mapsto(z-i) /(z+i)
\end{aligned}
$$

ist konform und induziert die Metrik

$$
d s^{2}:=\frac{1}{4} \operatorname{Im}(z)^{-2}|d z|^{2}
$$

auf $\mathbb{H}$. Die durch $d s$ gegeben Abstandsfunktion auf $\mathbb{H}$ bezeichnen wir ebenfalls mit $d(\cdot, \cdot)$. Fuchssche Gruppen können also auch als auf $\mathbb{H}$ operierend aufgefaßt werden. Der Konvergenzexponent $\delta$ einer Fuchsschen Gruppe $G$ ist durch

$$
\delta:=\inf \left\{s>0: \sum_{g \in G}(\cosh d(g(z), w))^{-s}<\infty\right\}, \quad z, w \in D
$$

definiert. Diese Definition ist unabhängig von $z$ und $w$. Die Gitterpunktzählfunktion einer Fuchsschen Gruppe $G$ definieren wir als

$$
N(a ; z, w):=\#\{g \in G: \cosh d(g(z), w) \leq a\} .
$$

Wir werden geometrisch endliche Fuchssche Gruppen betrachten. Diese sind gerade die endlich erzeugten Gruppen (in höheren Dimensionen gilt das nicht mehr !). Für Details sei auf Beardon [1] verwiesen. Gute Einführungen zum Thema Fuchssche Gruppen sind außerdem [30] und [25].

Es sollen nun noch zwei wichtige Notationen erklärt werden. Für eine Teilmenge $\mathfrak{D}$ des $\mathbb{R}^{m}$ schreiben wir

$$
f\left(x_{1}, \ldots, x_{n}\right)=O\left(g_{1}\left(x_{1}, \ldots, x_{n}\right)\right)+O\left(g_{2}\left(x_{1}, \ldots, x_{n}\right)\right)+\ldots+O\left(g_{m}\left(x_{1}, \ldots, x_{n}\right)\right)
$$

für $\left(x_{1}, \ldots, x_{n}\right) \in \mathfrak{D}$, wenn es eine Konstante $C>0$ und Funktionen $\widetilde{g}_{i}\left(x_{1}, \ldots, x_{n}\right)$, $i \in\{1, \ldots, m\}$ gibt, mit

$$
\frac{\left|\widetilde{g}_{i}\left(x_{1}, \ldots, x_{n}\right)\right|}{g_{i}\left(x_{1}, \ldots, x_{n}\right)} \leq C \quad \text { für alle }\left(x_{1}, \ldots, x_{n}\right) \in \mathfrak{D} \subset \mathbb{R}^{m},
$$

sodaß $f\left(x_{1}, \ldots, x_{n}\right)=\sum_{i=1}^{m} \widetilde{g}_{i}\left(x_{1}, \ldots, x_{n}\right)$ für alle $\left(x_{1}, \ldots, x_{n}\right) \in \mathfrak{D}$.

Wir schreiben

$$
f(x) \sim g(x), \quad \text { für } x \rightarrow \infty
$$


wenn

$$
\lim _{x \rightarrow \infty} \frac{f(x)}{g(x)}=1
$$

und

$$
f(x)=\Omega(g(x))
$$

wenn $\lim \sup _{x \rightarrow \infty} \frac{|f(x)|}{g(x)}>0$.

\subsection{Zur Geschichte des Gitterpunktproblems}

Eine Verbesserung der von Gauß durch einfache geometrische Überlegungen gewonnenen Abschätzung von $d(R)$ gelang erst 1906. Sierpinski [39] zeigte mit spektraltheoretischen Methoden

$$
d(R)=O\left(R^{2 / 3}\right)
$$

(siehe hierzu auch Landau [18], achter Teil, Kapitel 2 sowie l.c. Kapitel 2). Van der Corput [4] zeigte 1923, daß die Konstante 2/3 in der obigen Gleichung durch eine kleinere Zahl ersetzt werden kann. Der nächste Durchbruch gelang 1924 Littelwood und Walfisz [20]. Sie konnten

$$
d(R)=O\left(R^{37 / 56}\right)
$$

beweisen $(37 / 56=0,66071 \ldots)$. Die letzte Verbesserung geht auf Huxley [16] zurück, der 1993

$$
d(R)=O\left(R^{46 / 73}\right)
$$

zeigte $(46 / 73=0,63013 \ldots)$. Es wird vermutet, daß

$$
d(R)=O\left(R^{1 / 2+\epsilon}\right)
$$

für alle $\epsilon>0$. Numerische Resultate unterstützen diese Vermutung. Daß die Asymptotik nicht stärker ist, zeigen Hardy [9] und Landau [17] im Jahre 1915. Sie beweisen

$$
d(R)=\Omega\left(R^{1 / 2} \log ^{1 / 4} R\right)
$$

(siehe hierzu auch Landau [18] achter Teil, Kapitel 6).

Analog zum klassischen Kreisproblem kann man die Anzahl $N(a ; z, w)$ von Orbitpunkten $\{g(w): g \in G\}$ einer Fuchsschen Gruppe $G$ in der hyperbolischen Ebene in einem Kreisgebiet $\left\{z^{\prime} \in D: \cosh d\left(z, z^{\prime}\right) \leq a\right\}$ mit Mittelpunkt $z \in D$ bestimmen.

In der Literatur wird die Gitterpunktzählfunktion für Fuchssche Gruppen wahrscheinlich zum ersten mal von Delsarte [5] [6] behandelt. Er stellt die Zählfunktion für cokompakte Fuchssche Gruppen durch eine Summe dar, in der die konfluente hypergeometrische Funktion sowie die Eigenwerte und Eigenfunktionen der Gruppe auftreten. Diese Formel ist das Analogon zu der exakten Formel von Hardy für $\mathbb{Z}^{2}$. 
Die Asymptotik der Zählfunktion Fuchsscher Gruppen wird zum ersten mal 1959 von Huber in [11] untersucht. Für cokompakte Fuchssche Gruppen vom Geschlecht $g$ beweist er

$$
N(a ; z, w) \sim \frac{1}{2(g-1)} \cdot a \quad \text { für } \quad a \rightarrow \infty
$$

(siehe auch Buser [3], Kapitel 9, §4). Huber betrachtet dazu die Funktion

$$
K(s, z, w)=\sum_{g \in G}(\cosh d(z, g(w)))^{-s}, \quad \operatorname{Re}(s)>1,
$$

gewinnt durch die Spektralzerlegung des $G$-invarianten Kerns $K(s, z, w)$ mit $\operatorname{Re}(s)>1$ eine meromorphe Fortsetzung von $K(s, z, w)$ in $s$ auf ganz $\mathbb{C}$ und kann dann das WienerIkehara Theorem anwenden, um sein Resultat zu erhalten.

Die Arbeiten von Selberg [36], [37], [38] zur Spektraltheorie Fuchsscher Gruppen sind besonders auch im Zusammenhang mit dem Gitterpunktproblem von Bedeutung. Außer einer kurzen Fußnote in [36] auf Seite 62, die auf die Anwendbarkeit der gewonnenen Spektralzerlegung auf das Gitterpunktproblem hinweist, hat Selberg jedoch nichts zur Gitterpunktzählfunktion veröffentlicht.

Die Asymptotik der Zählfunktion mit Fehlerterm wird 1975 von Patterson [26] betrachtet. Er zeigt, daß für eine cofinite Fuchssche Gruppe mit $L^{2}$-Eigenfunktionen $\phi_{0}=$ $(\operatorname{area}(\mathbb{H} / G))^{-1 / 2}, \phi_{1}, \phi_{2}, \ldots$ und Eigenwerten $\lambda_{0}=0, \lambda_{1}, \lambda_{2}, \ldots$ (siehe Kapitel 3.1.2) und mit $s_{k}:=\frac{1}{2}+\sqrt{\frac{1}{4}-\lambda_{k}}$ für $\lambda_{k} \leq 1 / 4$ gilt, daß

$$
N(a ; z, w)=\frac{2 \pi}{\operatorname{area}(\mathbb{H} / G)} \cdot a+\sum_{1>s_{k}>3 / 4} 2 \sqrt{\pi} \frac{\Gamma\left(s_{k}-1 / 2\right)}{\Gamma\left(1+s_{k}\right)} \phi_{k}(z) \phi_{k}(w) a^{s_{k}}+O\left(a^{3 / 4}\right)
$$

(siehe auch Buser [3], Kapitel 9, §7). Günther [8] konnte den Exponenten 3/4 für cokompakte Gruppen auf 2/3 verbessern. Er verallgemeinerte die Aussage außerdem auf alle cokompakten Gruppen auf nicht kompakten symmetrischen Räumen vom Rang eins.

Fuchssche und $n$-dimensionale Kleinsche Gruppen mit Fundamentalbereichen mit unendlichem Flächeninhalt bzw. Volumen werden erstmals 1982 von Lax und Phillips [19] untersucht. Sie zeigen, daß eine geometrisch-endliche Fuchssche bzw. Kleinsche Gruppe $G$ mit Fundamentalbereichen von unendlichem Flächeninhalt bzw. Volumen stets nur endlich viele $L^{2}$-Eigenfunktionen $\phi_{1}, \phi_{2}, \ldots, \phi_{n}$ hat und benutzen Eigenschaften von Lösungen der Wellengleichung auf $\mathbb{H} / G$ und Sobolev Ungleichungen, um für die Gitterpunktzählfunktion von Fuchsschen Gruppen zu zeigen, daß

$$
N(a ; z, w)=\sum_{1 \geq s_{k}>\frac{1}{3}(1+\delta)} 2 \sqrt{\pi} \frac{\Gamma\left(s_{k}-1 / 2\right)}{\Gamma\left(1+s_{k}\right)} \phi_{k}(z) \phi_{k}(w) a^{s_{k}}+O\left((\log a)^{5 / 6} a^{\frac{1}{3}\left(1+s_{1}\right)}\right)
$$

wobei $\lambda_{1}, \ldots, \lambda_{n}$ die zu $\phi_{1}, \phi_{2}, \ldots, \phi_{n}$ gehörigen Eigenfunktionen seien und $s_{k}$ wie oben definiert sei. Vorausgesetzt ist dabei, daß es mindestens einen $L^{2}$-Eigenwert gibt. Das ist genau dann der Fall, wenn der Konvergenzexponent der Gruppe größer als 1/2 ist (bzw. für Kleinsche Gruppen in $n$-Dimensionen, wenn der Konvergenzexponent größer als 
$(n-1) / 2$ ist). Man beachte, daß $s_{1}$ gleich dem Konvergenzexponenten der Gruppe ist. Analoge Ergebnisse erhalten Lax und Phillips für Kleinsche Gruppen in $n$ Dimensionen.

Neben der Methode mit Spektraltheorie, die Asymptotik der Gitterpunktzählfunktion zu finden, gibt es einen Ansatz, der die Ergodentheorie des geodätischen Flusses heranzieht. Nicholls [24] beweist 1983 analog zu Hubers Resultat, daß für cofinite Kleinsche Gruppen in $n$-Dimensionen mit Fundamentalbereich vom Volumen $V$ gilt, daß

$$
N(a ; z, w) \sim \frac{2 \pi}{V} \cdot a \quad \text { für } \quad a \rightarrow \infty .
$$

Dieses Resultat ist in dem von Patterson [26] enthalten. Nicholls benutzt keine Spektraltheorie, sondern die Mischungseigenschaft des geodätischen Flusses auf dem Tangentialraum.

Da $n$-dimensionale Kleinsche Gruppen mit Konvergenzexponent $\delta \leq(n-1) / 2$ keine $L^{2}$-Eigenfunktionen besitzen, liefert die Spektraltheorie in diesem Fall keine befriedigenden Ergebnisse. Für eine geometrisch endliche Gruppe $G$ mit $\delta>(n-1) / 2$ erhält man über das Patterson Maß $\mu$ (siehe [28] und [30]) die $L^{2}$-Eigenfunktion $F$ zum kleinsten Eigenwert $\lambda_{1}=\delta(1-\delta)$ durch

$$
F(x)=\int P(x, \zeta)^{\delta} d \mu(\zeta)
$$

wobei $P(x, \zeta)$ der Poissonkern des entsprechenden Modells des hyperbolischen Raumes ist. Für die Details sei auf [30] und [25] verwiesen. Ist $\delta \leq(n-1) / 2$, so ist $F$-wie oben über das Patterson Maß definiert- immer noch eine Eigenfunktion des LaplaceBeltramioperators, ist jedoch nicht mehr quadratintegrabel. Man kann $F$ dann als die kleinste Resonanz auf $\mathbb{H} / G$ bezeichnen.

Für konvex-cokompakte Gruppen mit beliebigem $\delta$ (also insbesondere auch für $\delta \leq$ $(n-1) / 2)$ konnte Patterson [31] zeigen, daß es eine Konstante $c>0$ gibt, sodaß

$$
N(a ; z, w) \sim c \cdot F(z) F(w) \cdot a^{\delta} .
$$

Für den Beweis benutzt er die meromorphe Fortsetzung der Reihe (1.2.2) (die Reihe konvergiert hier für $\operatorname{Re}(s)>\delta$ ), die er über die meromorphe Fortsetzung des Resolventenkerns erhält. Die Fortsetzung des Resolventenkerns gelang 1987 Mazzeo und Melrose [22]. Um das Wiener-Ikehara Theorem anwenden zu können, muß im wesentlichen noch gezeigt werden, daß es auf der Abszisse $\{s \in \mathbb{C}: \operatorname{Re}(s)=\delta\}$ außer bei $\delta$ keine weiteren Pole gibt. Dies gelingt Patterson unter Verwendung der Ergodizität des Patterson Maßes.

Für geometrisch endliche Gruppen mit Spitzen zeigt B. O. Stratmann [40] über eine Verallgemeinerung des Sullivan Schatten-Lemmas für das Patterson Maß, daß

$$
N(a ; z, z)=O\left(a^{\delta}\right)
$$

Im Falle, daß $\delta<(n-1) / 2$, ist dieses Ergebnis in keinem der oben erwähnten enthalten. Abschätzungen für den Fehlerterm der Asymptotik der Zählfunktion wie in (1.2.3) sind für $\delta \leq(n-1) / 2$ nicht bekannt. 
Für cokompakte Gruppen gibt Huber [15] 1995 eine Abschätzung der Zählfunktion in der Form

$$
N(a ; z, z) \geq \frac{1}{2(g-1)} \cdot a-D(a) \quad \text { für } \quad a>A
$$

an, wobei er $A$ und $D$ explizit angibt. In $A$ und in die Funktion $D$ geht lediglich der Injektivitätsradius von $z$ ein.

Für cofinite Gruppen sind $\Omega$-Resultate bekannt. R. Phillips und Z. Rudnick definieren 1994 in [32] den führenden Term einer cofiniten Gruppe $G$ als

$$
\begin{aligned}
\Sigma(a, z):= & \frac{2 \pi}{\operatorname{area}(\mathbb{H} / G)} \cdot a+\sum_{1>s_{k}>1 / 2} 2 \sqrt{\pi} \frac{\Gamma\left(s_{k}-1 / 2\right)}{\Gamma\left(1+s_{k}\right)}\left|\phi_{k}(z)\right|^{2} a^{s_{k}} \\
& +8(\rho+2(\log 2-1)) a^{1 / 2} \sum_{s_{k}=1 / 2}\left|\phi_{k}(z)\right|^{2}
\end{aligned}
$$

mit $a=\cosh \rho$ und damit den Fehlerterm

$$
d(a):=N(a ; z, z)-\Sigma(a, z)
$$

(mit Notation wie oben). Für cokompakte Gruppen und Untergruppen von PSL $(2, \mathbb{Z})$ mit endlichem Index zeigen sie, daß

$$
d(a)=\Omega\left(a^{1 / 2} \log ^{1 / 4-\epsilon} \rho\right)
$$

für alle $\epsilon>0$ (wie oben ist $a=\cosh \rho$ ). Für beliebige cofinite Gruppen zeigen sie

$$
d(a)=\Omega\left(a^{1 / 2-\epsilon}\right)
$$

für alle $\epsilon>0$.

\subsection{Ergebnisse}

In folgendem Abschnitt sollen die Ergebnisse dieser Arbeit erläutert werden. Theorem $\mathrm{C}$ und D werden nicht im Detail wiedergegeben, da beide recht lang sind.

Als erstes Resultat ist Theorem A aus Kapitel 2 zu nennen. Es präzisiert Sierpinskis Ergebnis (siehe Abschnitt 1.2 Seite 7) zur Gitterpunktzählfunktion für das Gitter $\mathbb{Z}^{2}$ in dem Sinne, daß numerische Abschätzungen für den Restterm der Zählfunktion gegeben werden und verallgemeinert es außerdem auf beliebige Gitter. Eine Verallgemeinerung auf alle kristallographischen Gruppen in der euklidischen Ebene, allerdings mit einer etwas schwächeren Abschätzung für den Restterm, gaben schon Lax und Phillips in [19]. Für ein Gitter $Z$ betrachten wir die Gitterpunktzählfunktion $N(R, p)$ und den zugehörigen Restterm $d(R, p)$ (siehe Abschnitt 1.1). In Kapitel 2 wird folgendes Theorem bewiesen: 
Theorem A. Für ein Gitter $Z$ mit Eigenwerten $\lambda_{0}=0<\lambda_{1} \leq \lambda_{2} \leq \cdots$ (siehe Kapitel 2.1) und mit

$$
A:=\sqrt{\lambda_{1}+\lambda_{3}}, \quad B:=\sqrt{2} A^{2}\left(\lambda_{1}^{-\frac{3}{4}}+\frac{2}{5}\right)+4 \sqrt{2} A\left(\lambda_{1}^{-\frac{1}{4}}+\frac{1}{3}\right),
$$

gilt für $R \geq \operatorname{area}^{2}(T) \cdot \max \left\{\frac{\lambda_{1}^{\frac{3}{2}}}{1875}, 22\right\}$, daß

$$
|d(R, p)| \leq 24,1 \cdot \operatorname{area}^{\frac{1}{3}}(T) R^{\frac{2}{3}}+B R^{\frac{1}{2}}+\operatorname{area}^{-1}(T) .
$$

Insbesondere ist also

$$
d(R, p)=O\left(R^{\frac{2}{3}}\right) .
$$

Man beachte, daß die Abschätzung des Restterms unabhängig vom Basispunkt $p$ ist. In der mir bekannten Literatur wird -mit Ausnahme von [19]- ausschließlich der Fall $Z=$ $\mathbb{Z}^{2}$ behandelt. Die verwendete Methode (siehe Abschnitt 1.4) ist im wesentlichen dieselbe, die Huber in [15] verwendet, um eine untere Schranke für die Gitterpunktzählfunktion cokompakter Fuchsscher Gruppen zu erhalten. Als einfache Folgerung ergibt sich:

Korollar A. Für das Gitter $Z=\mathbb{Z}^{2}$ gilt für $R \geq 22$, daß

$$
|d(R)| \leq 24,1 \cdot R^{\frac{2}{3}}+90 \cdot R^{\frac{1}{2}}+1 .
$$

Das wichtigste Ergebnis dieser Arbeit ist in Theorem B aus Kapitel 3.2 formuliert. Kapitel 2 soll insbesondere dazu dienen, die Methode, welche zum Beweis von Theorem $\mathrm{B}$ verwendet wird, anhand einfacherer Gitter -wie denen in der euklidischen Ebene- zu erläutern. Anstatt eines euklidischen Gitters kann auch eine Fuchssche Gruppen in der hyperbolischen Ebene und deren Zählfunktion $N(a ; z, w)$ betrachtet werden (siehe Abschnitt 1.1). Von besonderem Interesse ist Theorem B für geometrisch endliche Gruppen mit einem Fundamentalbereich von unendlichem Flächeninhalt. In diesem Fall ist der Konvergenzexponent der Gruppe echt kleiner als Eins.

Theorem B. Es sei $G$ eine geometrisch endliche torsionsfreie Fuchssche Gruppe mit Konvergenzexponenten $\delta>1 / 2$, diskreten Eigenwerten $\lambda_{1}, \lambda_{2}, \ldots, \lambda_{n} \in[0,1 / 4)$ und zugehörigen normierten reellen Eigenfunktionen $\phi_{1}, \phi_{2}, \ldots, \phi_{n} \in L^{2}(D / G)$. Es sei $s_{k}:=$ $\frac{1}{2}+\sqrt{\frac{1}{4}-\lambda_{k}}$ und also $\delta=s_{1}$. Dann gilt

$$
N(a ; z, w)=\sum_{1 \geq s_{k}>\frac{1}{3}(1+\delta)} 2 \sqrt{\pi} \frac{\Gamma\left(s_{k}-1 / 2\right)}{\Gamma\left(1+s_{k}\right)} \phi_{k}(z) \phi_{k}(w) a^{s_{k}}+O\left(a^{\frac{1}{3}(1+\delta)}\right) .
$$

Für cofinite und cokompakte Fuchssche Gruppen (also $\delta=1$ ) ergibt sich das bekannte Ergebnis, daß der Restterm höchstens wie $a^{2 / 3}$ wächst (siehe Abschnitt 1.2). Lax und Phillips zeigen in [19] eine asymptotische Abschätzung wie die in Theorem B mit anderen Methoden. Ihr Fehlerterm ist von der Ordnung $O\left((\log a)^{5 / 6} a^{1 / 3(1+\delta)}\right)($ siehe 
Abschnitt 1.2 Gleichung (1.2.3) ). Theorem B liefert also ein etwas besseres Ergebnis. Der Beweis verallgemeinert die von Huber in [15] verwendete Methode auf geometrisch endliche Gruppen mit einem Fundamentalbereich von unendlichem Flächeninhalt. Es wird gezeigt, wie neben einer unteren Schranke für die Zählfunktion -wie Huber sie in [15] für cokompakte Fuchssche Gruppen erhält- auch eine obere Schranke der Zählfunktion angegeben werden kann. Die Methode benutzt die Spektraltheorie auf den zu den Fuchsschen Gruppen gehörigen Riemannschen Flächen. Im Fall von nicht cokompakten geometrisch endlichen Fuchsschen Gruppen gibt es, anders als bei cokompakten Gruppen, neben dem diskreten Spektrum noch einen kontinuierlichen Anteil am Spektrum. Wir benutzen die Spektraltheorie aus Maurin [21] Kapitel XVII $\S 8$ und $\S 9$, die schon Patterson in [29] auf Fuchsschen Gruppen angewendet hat. Anders als Huber, der seine Spektralzerlegungen als Summen über die Eigenfunktionen erhält, müssen wir mit einem Integral über ein Spektralmaß arbeiten.

Theorem $\mathrm{C}$ und $\mathrm{D}$ geben analog zu Theorem A numerische Abschätzungen für den Restterm aus Theorem B. Es sei hier direkt auf Abschnitt 3.3 verwiesen. Um obere und untere Schranken für den Restterm angeben zu können, wird zusätzliche Information über die Fuchssche Gruppe benötigt. Zum einen müssen wir uns auf Fuchssche Gruppen mit nur genau einem $L^{2}$-Eigenwert beschränken, da weitere $L^{2}$-Eigenwerte die Asymptotik entscheidend beeinflussen und die zugehörigen Eigenfunktionen als zusätzliche Konstanten eingehen, über die wir wenig wissen (siehe Theorem B). Zum anderen benötigen wir a priori eine untere Schranke für den Konvergenzexponenten, welche echt größer als $1 / 2$ ist.

In Kapitel 3.4 wird Theorem C dazu verwandt, für cofinite Fuchssche Gruppen einen Test für die Existenz eines (neben Null) weiteren $L^{2}$-Eigenwertes anzugeben.

Korollar B. Es sei $G$ eine cofinite Fuchssche Gruppe mit einem Fundamentalbereich vom Flächeninhalt $A$. Es sei $z \in D$ und

$$
\begin{gathered}
r:=\operatorname{inj}(z)>0 \\
\nu(r):= \begin{cases}\frac{5}{4} & \text { falls } \sinh ^{-2} \frac{r}{2}<\frac{5}{4}, \\
\sinh ^{-2} \frac{r}{2} & \text { falls } \sinh ^{-2} \frac{r}{2} \geq \frac{5}{4},\end{cases} \\
X_{0}:=\sqrt{\nu(r)-1 / 4} .
\end{gathered}
$$

Gibt es nun ein $a \in \mathbb{R}$ mit

$$
\begin{gathered}
a>\max \left\{6,0,6 \cdot C_{0}^{2 / 3}\right\}, \\
a>\left(C_{0}^{-2 / 3} \cdot \max \left\{7,8,1,6 \cdot X_{0}\right\}\right)^{2},
\end{gathered}
$$


so $d a \beta$ gilt, daß

$$
\begin{aligned}
N(a ; z, z)> & \frac{2 \pi}{A} a+15 \cdot\left(\frac{2 \pi}{A}\right)^{1 / 3} \cdot a^{2 / 3} \\
& +18 \cdot\left(\frac{2 \pi}{A}\right)^{-1 / 3} a^{1 / 3}+19 \cdot\left(\frac{2 \pi}{A}\right)^{-2 / 3} a^{1 / 6} \\
& +\left(1,5 \cdot \log \frac{2 \pi}{A}+0,8 \cdot \log a+3 \cdot \nu(r) \rho+27\right) \cdot a^{1 / 2}+\frac{3}{A},
\end{aligned}
$$

wobei $\rho>0$ durch $a=\cosh \rho$ definiert ist, dann hat der Laplace-Beltrami Operator auf $D / G$ neben dem Eigenwert Null einen weiteren Eigenwert $\lambda_{1}$ mit $0<\lambda_{1}<\frac{1}{4}$.

Es bezeichnet $\operatorname{inj}(z)$ den Injektivitätsradius von $z \in D$ (siehe Abschnitt 3.1 Seite 32).

Als zweite Anwendung gibt Korollar C die Möglichkeit, den Konvergenzexponenten und die erste Eigenfunktion einer geometrisch endlichen Fuchsschen Gruppe über ihre Zählfunktion zu approximieren. Die Genauigkeit dieser Approximationen hängt von der Größe des Kreises ab, in dem die Gitterpunkte ausgezählt werden.

Korollar C. Es sei $G$ eine geometrisch endliche Fuchssche Gruppe, die nur genau einen Eigenwert kleiner als $1 / 4$ hat. Für den Konvergenzexponenten $\delta$ von $G$ gebe es eine Zahl $\delta_{0}>1 / 2$, soda $\beta \delta \delta_{0}$. Die normierte Eigenfunktion zum kleinsten Eigenwert $\delta(1-\delta)$ sei $\phi$. Es sei $z \in D, r:=\operatorname{inj}(z)>0$ und $\nu, X_{0}$ wie in Theorem $C$,

$$
C:=2 \sqrt{\pi} \frac{\Gamma(\delta-1 / 2)}{\Gamma(1+\delta)} \phi^{2}(z)
$$

Weiter sei

$$
f(x):=\log C+\delta x .
$$

Dann gilt für alle $C_{0}>0$ und mit $\rho>0$ definiert durch $a=\cosh \rho$ und für alle $a \in \mathbb{R}$ mit

$$
\begin{gathered}
a>\max \left\{6,0,6 \cdot C_{0}^{2 / 3}\right\}, \\
a>\left(C_{0}^{-2 / 3} \cdot \max \left\{7,8,1,6 \cdot X_{0}\right\}\right)^{\frac{2}{2 \delta_{0}-1}}
\end{gathered}
$$

und mit

$$
\begin{aligned}
B_{o}(a):= & \log \left\{N(a ; z, z)+7,8 \cdot C_{0}^{1 / 3} a^{2 / 3}+3,2 \cdot C_{0}^{-1 / 3} a^{1 / 3\left(2-\delta_{0}\right)}\right. \\
& \left.+6,7 \cdot C_{0}^{-2 / 3} a^{1 / 6\left(5-4 \delta_{0}\right)}+\left(\log C_{0}+\frac{1}{2} \log a+2 \cdot \nu(r) \rho+19\right) \cdot a^{1 / 2}\right\} \\
& -\log \left\{1-3,9 \cdot C_{0}^{-2 / 3} a^{1 / 3\left(1-2 \delta_{0}\right)}-\frac{2}{\pi}\left(2+\left(\delta_{0}-\frac{1}{2}\right)^{-1}\right) a^{\left(1-2 \delta_{0}\right)}\right. \\
& \left.-1,5 a^{-\delta_{0}}-\frac{3}{\pi} a^{-\delta_{0}}\right\}
\end{aligned}
$$




$$
\begin{aligned}
B_{u}(a):= & \log \left\{N(a ; z, z)-11,1 \cdot C_{0}^{1 / 3} a^{2 / 3}-18 \cdot C_{0}^{-1 / 3} a^{1 / 3\left(2-\delta_{0}\right)}\right. \\
& -19 \cdot C_{0}^{-2 / 3} a^{1 / 6\left(5-4 \delta_{0}\right)} \\
& \left.-\left(1,5 \cdot \log C_{0}+0,8 \cdot \log a+3 \cdot \nu(r) \rho+27\right) \cdot a^{1 / 2}\right\} \\
& -\log \left(1+3,9 \cdot C_{0}^{-2 / 3} a^{1 / 3\left(1-2 \delta_{0}\right)}+\frac{3}{2 \pi} a^{-\delta_{0}}\right)
\end{aligned}
$$

$d a \beta$

$$
B_{u}(a) \leq f(\log a) \leq B_{o}(a) .
$$

Erfüllen $a_{1}$ und $a_{2}$ mit $a_{1}<a_{2}$ die oben an a gestellten Bedingungen, so gilt für den Konvergenzexponenten $\delta$ von $G$, daß

$$
\frac{B_{u}\left(a_{2}\right)-B_{o}\left(a_{1}\right)}{\log a_{2}-\log a_{1}} \leq \delta \leq \frac{B_{o}\left(a_{2}\right)-B_{u}\left(a_{1}\right)}{\log a_{2}-\log a_{1}}
$$

und für $C$ wie oben definiert, daß

$$
\frac{\log \left(a_{2}\right) B_{u}\left(a_{1}\right)-\log \left(a_{1}\right) B_{o}\left(a_{2}\right)}{\log a_{2}-\log a_{1}} \leq C \leq \frac{\log \left(a_{2}\right) B_{o}\left(a_{1}\right)-\log \left(a_{1}\right) B_{u}\left(a_{2}\right)}{\log a_{2}-\log a_{1}} .
$$

In Appendix C ist ein Computerprogramm angegeben, das die Gitterpunktzählfunktion von Heckegruppen bestimmt. Die Heckegruppe $\Gamma_{\mu}$ ist die von den beiden in der oberen Halbebene operierenden Isometrien

$$
z \mapsto z+\mu, z \mapsto-\frac{1}{z}
$$

frei erzeugte Gruppe. Für $\mu>2$ hat $\Gamma_{\mu}$ einen Fundamentalbereich von unendlichem Flächeninhalt. Ein Ergebnis von Beardon gibt uns die Möglichkeit, eine untere Schranke für den Konvergenzexponenten zu bestimmen. Phillips und Sarnak beweisen, daß die Heckegruppe $\Gamma_{\mu}$ für $\mu \geq 2$ nur genau einen $L^{2}$-Eigenwert besitzt (siehe Abschnitt 3.4 Seite 53). Als Beispiel betrachten wir die Heckegruppe mit $\mu=2,4$. Der Computer liefert uns für $\Gamma_{2,4}$

$$
\begin{aligned}
& N\left(10^{12} ; 2 i, 2 i\right)=23.383 .324 .507 \\
& N\left(10^{14} ; 2 i, 2 i\right)=1.097 .048 .219 .243 .
\end{aligned}
$$

Es wurde auf einer SGI O2 gerechnet. Die Rechenzeit betrug etwa eine Woche. Aus Korollar C folgt nun mit inj $(2 i)>\log 2$ für den Konvergenzexponenten $\delta$ von $\Gamma_{2,4}$ :

$$
0,80 \leq \delta \leq 0,87
$$

In [23] gibt McMullen einen Algorithmus an, mit dem man die Hausdorffdimension der Limesmengen Kleinscher- und Fuchsscher Gruppen, sowie der Juliamengen rationaler Abbildungen bestimmen kann. Er schätzt die Konvergenzgeschwindigkeit seines Verfahrens ab und kommt zu dem Ergebnis, daß er $O(N)$ Rechenschritte benötigt, um die 
Hausdorffdimension auf $N$ Stellen genau zu berechnen. Bei jedem Rechenschritt muß für eine bestimmte Übergangsmatrix $T$ die Gleichung

$$
\lambda\left(T^{\alpha}\right)=1
$$

gelöst werden, wobei $\lambda(A)$ für den Spektralradius der Matrix $A$ steht, und $T^{\alpha}$ die Matrix bezeichnet $\left(\left(t_{i, j}\right)^{\alpha}\right)_{i, j}$ bezeichnet, für $T=\left(t_{i, j}\right)_{i, j}$. Die Lösung für $\alpha$ ist dann eine Approximation der zu bestimmenden Hausdorffdimension. Will man diese Gleichung numerisch lösen, so kann man mit dem Perron-Frobenius Theorem durch Iteration von $T^{\alpha}$ den Wert von $\lambda\left(T^{\alpha}\right)$ bestimmen und die Gleichung $\lambda\left(T^{\alpha}\right)=1$ mit dem Newton Verfahren lösen. Der Fehler, der dabei gemacht wird, läßt sich schwer exakt abschätzen, und so müssen die mit McMullens Algorithmus bestimmten Werte für Hausdorffdimensionen als empirisch angesehen werden. Im Gegensatz dazu bietet Korollar C unter der Voraussetzung, daß die Gitterpunktzählfunktion der zu untersuchenden Gruppe zuverlässig für große Radien bestimmt werden kann, die Möglichkeit, rigorose untere und obere Schranken für die Hausdorffdimension der Limesmenge, bzw. für den Konvergenzexponenten einer geometrisch endlichen Fuchsschen Gruppe, anzugeben.

\subsection{Die verwendete Methode}

Es soll nun die Methode erläutert werden, die sowohl in Kapitel 2 als auch in Kapitel 3 verwendet wird, um respektive die Gitterpunktzählfunktion für euklidische Gitter und Fuchssche Gruppen abzuschätzen. Da die Anwendung der Methode auf das klassische Kreisproblem weniger technisch ist als die auf das Gitterpunktproblem in der hyperbolische Ebene, soll sie hier auch anhand des klassischen Kreisproblems erklärt werden. Alle Details finden sich in Kapitel 2. Im wesentlichen ist die Methode die von Huber in [15] auf cokompakte Fuchsschen Gruppen angewendete.

Für ein Gitter $Z$ aus $\mathbb{R}^{2}$-man denke z.B. am $\mathbb{Z}^{2}$ - betrachten wir die Gitterpunktzählfunktion $N(R, p)$ und den zugehörigen Restterm $d(R, p)$ für $R \geq 0$ und $p \in \mathbb{R}^{2}$ (siehe Abschnitt 1.1). Wie wir bereits sahen, gilt für den Restterm für festes $p$, daß

$$
d(R, p)=O(R) .
$$

Unser Ziel ist es, diese Abschätzung zu verbessern. Dazu betrachten wir die Zählfunktion nicht wie oben nur als Funktion von $R$ für einen festen Punkt $p$, sondern richten unsere Aufmerksamkeit auf die Abhängigkeit der Zählfunktion vom Basispunkt $p$.

Als Funktion von $p$ ist die Zählfunktion "sehr" unstetig. Sie ist auf Bereichen konstant, welche durch das Schneiden von Kreisen vom Radius $R$ um eine Auswahl von Gitterpunkten entstehen. Je größer $R$ wird, desto feiner wird die Unterteilung eines Fundamentalbereiches von $Z$ in solche Bereiche ausfallen. In Abbildung 1.1 sind die Bereiche aus $[0,1] \times[0,1]$ dargestellt, auf denen die Zählfunktion $N(R, \cdot)$ des Gitters $\mathbb{Z}^{2}$ konstant ist. Die erste Abbidung zeigt $N(R, \cdot)$ für $R=1,3$ die zweite für $R=1,9$ und letzt für $R=18,5$. Für die Radien 1,3 und 1,9 ist in den Bereichen der Zahlenwert, den die Zählfunktion dort annimmt, eingetragen. Die Komplexität der Zählfunktion für feste große Radien $R$ zeigt uns die letzte Abbildung. 

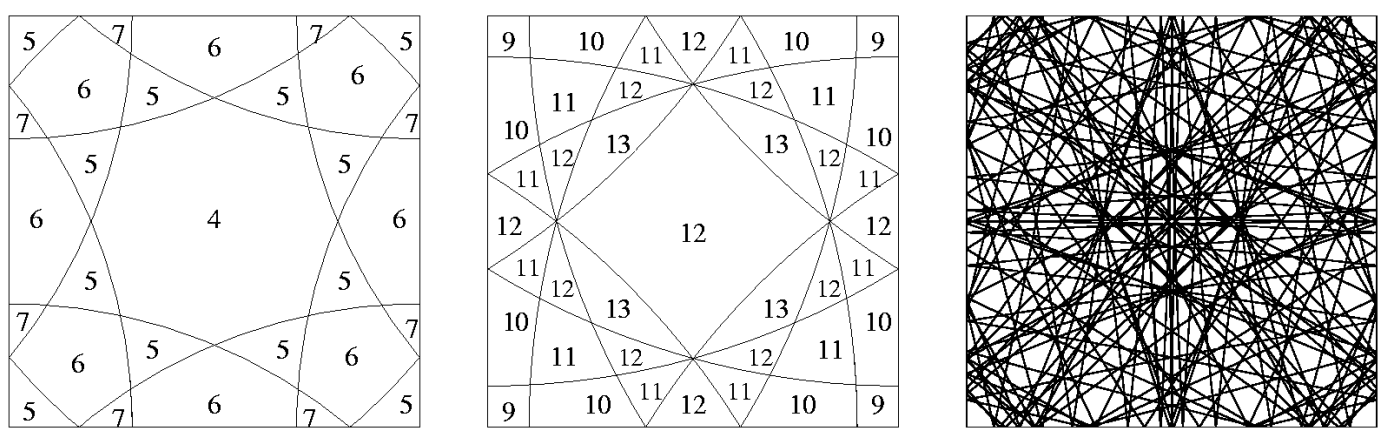

Abbildung 1.1.: Bereiche auf denen die Zählfunktion konstant ist

Wir können den Restterm $d(R, p)$ gleichmäßig für alle $p$ abschätzen, wenn wir die "Schwankungen" von $d(R, \cdot)$ (also des Restterms als Funktion von $p$ ) quantifizieren können. Ein probates Mittel hierzu liefert uns die Spektraltheorie. Um die Methoden der Spektraltheorie wirkungsvoll anwenden zu können, müssen wir die Gitterpunktzählfunktion zuerst glätten. Definieren wir

$$
k_{0}(r):=\left\{\begin{array}{lll}
1 & \text { für } \quad 0 \leq r \leq R \\
0 & \text { für } \quad r>R
\end{array}\right.
$$

dann kann auch

$$
N(R, p)=\sum_{\omega \in Z} k_{0}(\|\omega-p\|)
$$

geschrieben werden. $\operatorname{Um~} N(R, \cdot)$ zu glätten, ersetzen wir die Funktion $k_{0}$ durch eine differenzierbare Funktion $k_{R}$, welche neben $R$ noch von einem weiteren Parameter $h$ abhängt, welcher als Maß für die Glättung angesehen werden kann. Dieser Parameter wird später für jedes $R$ so gewählt werden, daß ein optimales Ergebnis erzielt wird. In Kapitel 2 wird die Funktion $k_{R}$ noch ein wenig anders definiert, um gewisse Konstanten klein zu halten. Wir definieren hier

$$
b(x):=2 x^{3}-3 x^{2}+1
$$

und damit für $h>0$ die Funktion $k_{R} \in C_{0}^{1}[0, \infty)$ durch

$$
k_{R}(r)= \begin{cases}1 & \text { für } 0 \leq r \leq R-h, \\ b\left(\frac{r-R+h}{h}\right) & \text { für } R-h<r \leq R, \\ 0 & \text { für } r>R\end{cases}
$$

(siehe Abbildung 1.2).

Die beiden geglätteten Zählfunktionen

$$
K^{b}(R, p):=\sum_{\omega \in Z} k_{R}(\|\omega-p\|) \quad \text { und } \quad K^{\sharp}(R, p):=\sum_{\omega \in Z} k_{R+h}(\|\omega-p\|)
$$

geben uns obere und untere Abschätzungen für die ursprüngliche Gitterpunktzählfunktion

$$
K^{b}(R, p) \leq N(R, p) \leq K^{\sharp}(R, p) .
$$




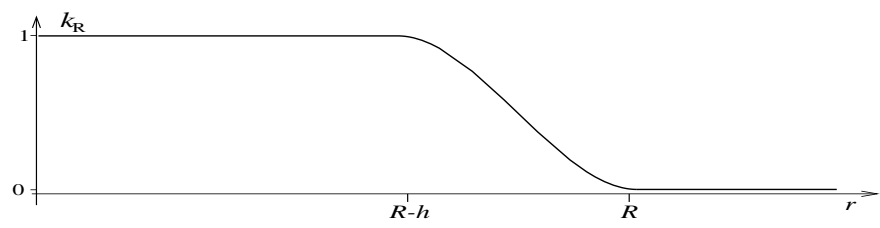

Abbildung 1.2.: Die Glättungsfunktion

Diese glatteren Funktionen $K^{\text {b }}$ und $K^{\sharp}$ werden nun in $Z$-translationsinvariante Eigenfunktionen des Laplaceoperators zerlegt, um ein Maß für ihre Schwankung zu erhalten. Der Laplaceoperator auf $T$ hat ein rein diskretes Spektrum. Die Eigenwerte seien mit $\lambda_{0}=0, \lambda_{1}, \lambda_{2}, \ldots$ bezeichnet und die zugehörigen Eigenfunktionen mit $\phi_{0}, \phi_{1}, \phi_{2}, \ldots$ (siehe Seite 20). Da $K^{\sharp}$ und $K^{b}$ stetig sind, bekommen wir absolut konvergente Reihen

$$
\begin{aligned}
K^{\sharp}(R, p) & =\frac{1}{\operatorname{area}(T)} \sum_{i=0}^{\infty} g_{k_{R}}\left(\lambda_{i}\right) \phi_{i}(p) \quad \text { und } \\
K^{b}(R, p) & =\frac{1}{\operatorname{area}(T)} \sum_{i=0}^{\infty} g_{k_{R+h}}\left(\lambda_{i}\right) \phi_{i}(p)
\end{aligned}
$$

(siehe Theorem 2.1.3 auf Seite 22). Der Ausdruck area ( $T$ ) bezeichnet den Flächeninhalt von $T$. Die Koeffizienten können explizit berechnet werden

$$
g_{k}(\lambda):=2 \pi \int_{0}^{\infty} k(r) J_{0}(\sqrt{\lambda} r) r d r
$$

wobei $J_{0}$ die Besselfunktion nullter Ordnung ist (siehe Seite 21) . Mit Hilfe dieser Darstellung können wir die Koeffizienten abschätzen. Es gilt

$$
\left|g_{k_{R}}(\lambda)\right|=O\left(R^{1 / 2} \lambda^{-3 / 4}\right)
$$

und

$$
\left|g_{k_{R}}(\lambda)\right|=O\left(R^{1 / 2} h^{-1} \lambda^{-5 / 4}\right)
$$

(siehe Lemma 2.2.1 und 2.2.2 auf Seite $24 \mathrm{ff}$.). Die Abschätzung (1.4.3) ist gut in $R$, jedoch kann mit ihr nicht die absolute Konvergenz der Summe aus (1.4.2) gezeigt werden. In die Abschätzung (1.4.4) geht die spezielle Form der Funktion $k_{R}$ ein. Wesentlich ist dabei, daß $k_{R}$ auf einem Intervall der Länge $h$ von 1 auf 0 abfällt und differenzierbar ist (die spezielle Wahl von $b$ ist nur in Hinsicht auf diese beiden Eigenschaften wichtig). Denken wir uns $h$ in Abhängigkeit von $R$ gewählt, so wird die Abschätzung (1.4.4), wenn $h$ mit wachsendem $R$ kleiner wird, schlechter (als $R^{1 / 2}$ ) in $R$. Der Fehler, den $h$ im führenden Term (1.4.6) verursacht, wird mit abnehmendem $h$ jedoch kleiner. Diese beiden Phänomene müssen am Ende ins Gleichgewicht gebracht werden.

Wir benötigen noch die folgende Abschätzung

$$
\sum_{\lambda_{i}<X}|\phi(p)|=O(X)
$$


(siehe Proposition 2.1.2 auf Seite 21). Sie gewinnt man durch ein Packungsargument wie oben, angewandt auf das zu $Z$ duale Gitter. Mit

$$
\operatorname{Rest}(R, h):=\sum_{\lambda_{i}>0}\left|g_{k}(\lambda)\right|
$$

folgt aus (1.4.1), (1.4.2) und

$$
g_{k}(0)=\frac{\pi}{\operatorname{area}(T)} R^{2}+O(R h),
$$

$\mathrm{daß}$

$$
N(R, p)=\frac{2 \pi}{\operatorname{area}(T)} R^{2}+O(R h)+O(\operatorname{Rest}(R, h)) .
$$

Um nun Rest $(R, h)$ abzuschätzen, benutzen wir sowohl (1.4.3), als auch (1.4.4), indem wir einen zusätzlichen Parameter $s>0$ einführen, sodaß

$$
\begin{gathered}
\operatorname{Rest}(R, h)=\Sigma^{\prime}(s)+\Sigma^{*}(s) \quad \text { mit } \\
\Sigma^{\prime}(s):=\sum_{0<\lambda_{i}<s}\left|g_{k}(\lambda)\right|, \quad \Sigma^{*}(s):=\sum_{\lambda_{i}>s}\left|g_{k}(\lambda)\right| .
\end{gathered}
$$

Für die Abschätzung der ersten Summe benutzen wir (1.4.3) und für die zweite Summe (1.4.4). An dieser Stelle wird außerdem noch (1.4.5) benötigt. Es ergibt sich

$$
\Sigma^{\prime}(s)=O\left(R^{1 / 2} s^{1 / 4}\right), \quad \Sigma^{*}(s)=O\left(R^{1 / 2} h^{-1} s^{-1 / 4}\right)
$$

(siehe Seite 25 ff.) und zusammen mit (1.4.7) also

$$
N(R, p)=\frac{\pi}{\operatorname{area}(T)} R^{2}+O(R h)+O\left(R^{1 / 2} s^{1 / 4}\right)+O\left(R^{1 / 2} h^{-1} s^{-1 / 4}\right) .
$$

Die optimale Wahl für $h$ und $s$ ist

$$
h=R^{-1 / 3} \quad \text { und } \quad s=R^{2 / 3} .
$$

Mit dieser Wahl erhalten wir unser Endergebnis

$$
N(R, p)=\frac{\pi}{\operatorname{area}(T)} R^{2}+O\left(R^{2 / 3}\right) .
$$

In Kapitel 2 wird genau nach diesem Muster vorgegangen. Allerdings werden die Konstanten, die hier in den $O$-Termen verschwinden, explizit berechnet. Im Falle Fuchsscher Gruppen wird analog verfahren. An die Stelle einer Summe über $L^{2}$-Eigenfunktionen tritt hier ein Integral über ein Spektralmaß. Um eine Abschätzung wie (1.4.5) zu erhalten, muß ein anderer Weg als im euklidische Fall gegangen werden. Die Spektralfunktion drückt sich nicht als ein Integral über das Produkt einer Besselfunktion nullter Ordnung und dem Kern aus. An die Stelle der Besselfunktionen treten nun Legendrefunktionen. Das Appendix A ist der Untersuchung ihrer asymptotischen Eigenschaften gewidmet. Im Unterschied zu den euklidischen Gittern muß der Parameter $h$ im Falle Fuchsschen Gruppen mit dem Radius des Kreises, in dem die Orbitpunkt gezählt werden, wachsen. 


\section{Das klassische Kreisproblem}

Um die in dieser Arbeit verwendete Methode zum Zählen von Gitterpunkten anhand eines einfachen Beispiels zu demonstrieren, soll sie zuerst auf das klassische Kreisproblem angewendet werden. Das von Gauß formulierte klassische Kreisproblem stellt die Frage nach der Anzahl von Punkten aus einem Gitter $Z$ in einem Kreis mit großem Radius. Für das Gitter $Z$ betrachen wir die Gitterpunktzählfunktion $N(R, p)$ und den zugehörigen Restterm $d(R, p)$ (siehe Abschnitt 1.1). In diesem Kapitel werden wir das folgende Theorem beweisen:

Theorem A. Für ein Gitter $Z$ mit Eigenwerten $\lambda_{0}=0<\lambda_{1} \leq \lambda_{2} \leq \cdots$ (siehe Kap. 2.1) und mit

$$
A:=\sqrt{\lambda_{1}+\lambda_{3}}, \quad B:=\sqrt{2} A^{2}\left(\lambda_{1}^{-\frac{3}{4}}+\frac{2}{5}\right)+4 \sqrt{2} A\left(\lambda_{1}^{-\frac{1}{4}}+\frac{1}{3}\right),
$$

gilt für $R \geq \operatorname{area}^{2}(T) \cdot \max \left\{\frac{\lambda_{1}^{\frac{3}{2}}}{1875}, 22\right\}, d a \beta$

$$
|d(R, p)| \leq 24,1 \cdot \operatorname{area}^{\frac{1}{3}}(T) R^{\frac{2}{3}}+B R^{\frac{1}{2}}+\operatorname{area}^{-1}(T) .
$$

Insbesondere ist also

$$
d(R, p)=O\left(R^{\frac{2}{3}}\right) .
$$

Daraus ergibt sich das folgende Korollar.

Korollar A. Für das Gitter $Z=\mathbb{Z}^{2}$ gilt für $R \geq 22$, daß

$$
|d(R, p)| \leq 24,1 \cdot R^{\frac{2}{3}}+90 \cdot R^{\frac{1}{2}}+1 .
$$

\subsection{Die Spektraltheorie euklidischer Gitter}

Es sei $Z$ das durch die linear unabhängigen Vektoren $v_{1}$ und $v_{2}$ definierte Gitter und $A=\left(v_{1}^{t}, v_{2}^{t}\right)$. Das zu $Z$ duale Gitter $Z^{\prime} \subset \mathbb{R}^{2}$ sei durch die Punkte

$$
\omega^{\prime}=k A^{-1} \quad k \in \mathbb{Z}^{2}
$$

definiert. Es sei $f$ eine unter $Z$-Translation invariante Funktion auf $\mathbb{R}^{2}$, die als Funktion auf $T=\mathbb{R}^{2} / Z$ aufgefaßt, in $L^{2}(T)$ liegt. Die Funktion $f(A \cdot): \mathbb{R}^{2} \rightarrow \mathbb{C}^{2}$ ist in beiden 
Koordinaten periodisch mit Periode eins, und wir können $f(A x)$ in eine Fourierreihe entwickeln:

$$
f(A x)=\sum_{k \in \mathbb{Z}^{2}} a_{k} \exp (2 \pi i k x)
$$

mit

$$
a_{k}=\int_{[0,1)^{2}} f(A x) \exp (-2 \pi i k x) d x .
$$

Dies ist aber äquivalent zu

$$
f(x)=\sum_{\omega^{\prime} \in Z^{\prime}} a_{\omega^{\prime}} \exp \left(2 \pi i \omega^{\prime} x\right)
$$

mit

$$
a_{\omega^{\prime}}=\operatorname{area}(T)^{-1} \int_{T} f(x) \exp \left(-2 \pi i \omega^{\prime} x\right) d x .
$$

Die Funktion $\exp \left(2 \pi i \omega^{\prime} x\right)$ mit $\omega^{\prime} \in Z^{\prime}$ ist eine unter $Z$-translationinvariante Eigenfunktion des negativen Laplaceoperators $-\Delta=-\left(\frac{\partial^{2}}{\partial x_{1}^{2}}+\frac{\partial^{2}}{\partial x_{2}^{2}}\right)$ mit Eigenwert $4 \pi^{2}\left\|\omega^{\prime}\right\|^{2}$. Es gibt eine Anordnung der Elemente aus $Z^{\prime}$, sodaß

$$
Z^{\prime}=\left\{\omega_{0}^{\prime}, \omega_{1}^{\prime}, \cdots\right\}, \quad \text { mit } \quad\left\|\omega_{i}^{\prime}\right\| \leq\left\|\omega_{j}^{\prime}\right\| \quad \text { für } \quad i<j .
$$

Damit definieren wir

$$
\begin{aligned}
\phi_{i}(x) & :=\exp \left(2 \pi i \omega_{i}^{\prime} x\right), \\
\lambda_{i} & :=4 \pi^{2}\left\|\omega_{i}^{\prime}\right\|^{2} .
\end{aligned}
$$

Dann gilt

$$
\lambda_{0}=0<\lambda_{1} \leq \lambda_{2} \leq \ldots
$$

Das obige Resultat kann somit folgendermaßen formuliert werden:

Theorem 2.1.1. Für alle $f \in L^{2}(T)$ gibt es eine Spektralzerlegung (die in der $L^{2}$-Norm konvergiert):

$$
f(x)=\sum_{i} a_{i} \phi_{i}(x)
$$

mit

$$
a_{i}:=\operatorname{area}(T)^{-1} \int_{T} f(x) \overline{\phi_{i}(x)} d x .
$$

Es sei $k$ eine reelle, meßbare Funktion auf $\mathbb{R}^{+}$mit kompaktem Träger. Durch

$$
K(p)=\sum_{\omega \in Z} k(\|\omega-p\|)
$$

wird eine $Z$-invariante Funktion definiert. Wir wollen nun $K$ in Eigenfunktionen des Laplaceoperators entwickeln. Dazu gehen zu Polarkoordinaten $p=(r \cos \varphi, r \sin \varphi)$ über. Für die Metrik $d s^{2}$, das Flächenelement $d A$ und den Laplace-Operator $\Delta$ gilt

$$
d s^{2}=r d \varphi^{2}+d r^{2},
$$




$$
\begin{gathered}
d A=r d \varphi d r, \\
\Delta=r^{-2} \partial_{\varphi}^{2}+\partial_{r}^{2}+r^{-1} \partial_{r} .
\end{gathered}
$$

Nun soll der $i$-te Koeffizient berechnet werden.

$$
\begin{aligned}
\int_{T} K(p) \overline{\phi_{i}(p)} d A(p) & =\sum_{\omega \in Z} \int_{F} k(\|\omega-p\|) \overline{\phi_{i}(p)} d A(p) \\
& =\int_{\mathbb{R}^{2}} k(\|p\|) \overline{\phi_{i}(p)} d A(p) \\
& =\int_{0}^{\infty} k(r) \int_{0}^{2 \pi} \overline{\phi_{i}(p(r, \varphi))} d \varphi r d r .
\end{aligned}
$$

Mit

$$
H_{i}(r):=\int_{0}^{2 \pi} \phi_{i}(p(r, \varphi)) d \varphi
$$

folgt durch Integration von $-\left(\Delta \phi_{i}\right)(p)=\lambda_{i} \phi_{i}(p)$ über $\varphi$, daß

$$
r H_{i}^{\prime \prime}(r)+H_{i}^{\prime}(r)+\lambda r H_{i}(r)=0 .
$$

Diese Differentialgleichung hat mit der Anfangsbedingung $H_{i}(0)=C$ mit $C>0$ die Lösung $C J_{0}(\sqrt{\lambda} r)$, wobei $J_{0}$ die Besselfunktion nullter Ordnung ist (siehe Watson [41]). Wegen

$$
\lim _{r \searrow 0} \int_{0}^{2 \pi} \phi_{i}(p(r, \varphi)) d \varphi=2 \pi
$$

gilt dann

$$
H_{i}(r)=2 \pi J_{0}(\sqrt{\lambda} r)
$$

Mit der Definition

$$
g_{k}(\lambda):=2 \pi \int_{0}^{\infty} k(r) J_{0}(\sqrt{\lambda} r) r d r
$$

folgt

$$
\int_{T} K(p) \overline{\phi_{i}(p)} d A(p)=g_{k}\left(\lambda_{i}\right)
$$

Wir wollen nun

$$
N_{\lambda}(X):=\#\left\{i: \lambda_{i} \leq X\right\}
$$

nach oben abschätzen. Das kann mit einem einfachen Packungsargument für das duale Gitter geschehen.

Proposition 2.1.2. Es sei $d:=\sqrt{\lambda_{1}+\lambda_{3}}$, dann gilt

$$
N_{\lambda}(X) \leq \frac{\operatorname{area}(T)}{4 \pi}(\sqrt{X}+d)^{2} .
$$


Beweis. Es ist

$$
N_{\lambda}(X)=\left\{\omega^{\prime} \in Z^{\prime}:\left\|\omega^{\prime}\right\| \leq \frac{\sqrt{X}}{2 \pi}\right\} \leq \pi \text { area }(T)\left(\frac{\sqrt{X}}{2 \pi}+\operatorname{diam}\left(\mathbb{R}^{2} / Z^{\prime}\right)\right)^{2} .
$$

Mit $\operatorname{diam}\left(\mathbb{R}^{2} / Z^{\prime}\right)=\sqrt{\left\|\omega_{1}^{\prime}\right\|^{2}+\left\|\omega_{3}^{\prime}\right\|^{2}}=\frac{1}{2 \pi} \sqrt{\lambda_{1}+\lambda_{3}}$ folgt die Behauptung.

Aus Theorem 2.1.1 und Proposition 2.1.2 folgt

Theorem 2.1.3. Es sei $k$ eine reelle, stetige Funktion auf $\mathbb{R}^{+}$mit kompaktem Träger und $g_{k}(\lambda)=O\left((1+\lambda)^{\beta}\right)$ für ein $\beta>1$. Für

$$
K(p):=\sum_{\omega \in Z} k(\|\omega-p\|)
$$

gilt dann

$$
K(p)=\frac{1}{\operatorname{area}(T)} \sum_{i} g_{k}\left(\lambda_{i}\right) \phi_{i}(p)
$$

und die Summe konvergiert absolut.

Beweis. Theorem 2.1.1 gibt uns die Konvergenz im $L^{2}$-Sinne, und aus Proposition 2.1.2 folgt

$$
\sum_{i>X}\left|g_{k}\left(\lambda_{i}\right) \phi_{i}(p)\right|=O\left(X^{1-\beta}\right)
$$

also die absolute Konvergenz. Mit der Stetigkeit von $K$ folgt die Behauptung.

\subsection{Abschätzungen der Gitterpunktzählfunktion.}

Es sei $Z$ ein Gitter auf $\mathbb{R}^{2}$ und

$$
k_{0}(r):=\left\{\begin{array}{lll}
1 & \text { für } \quad 0 \leq r \leq R \\
0 & \text { für } \quad r>R
\end{array}\right.
$$

Dann gilt für die Gitterpunktzählfunktion $N(R, p):=\#\{\omega \in Z:\|\omega-p\| \leq R\}$, daß

$$
N(R, p)=\sum_{\omega \in Z} k_{0}(\|\omega-p\|)
$$

Die formale Entwicklung von $N(R, p)$ nach den Eigenfunktionen des Laplaceoperators sieht wie folgt aus:

$$
\frac{\pi R^{2}}{\operatorname{area}(T)}+\frac{1}{\operatorname{area}(T)} \sum_{i=1}^{\infty} \frac{2 \pi R}{\sqrt{\lambda_{i}}} J_{1}\left(\sqrt{\lambda_{i}} R\right) \phi_{i}(p) .
$$

Hier ist $J_{1}$ die Besselfunktion erster Ordnung. Die Voraussetzungen von Theorem 2.1.3 sind jedoch für $k_{0}$ nicht erfüllt, und es kann nicht auf absolute Konvergenz der Reihe 
2.2.1 geschlossen werden. Für $Z=\mathbb{Z}^{2}$ und $p=0$ geben Hardy und Landau einen Beweis für die gleichmäßige Konvergenz von 2.2.1 gegen $N(R, 0)$ (siehe z.B. [18]). Um absolute Konvergenz zu bekommen, müssen wir $k_{0}$ durch eine "glattere" Funktion approximieren. Wir definieren hierzu wie Huber in [15]

$$
\begin{gathered}
b(x):=\int_{x}^{1} y^{\epsilon}(1-y)^{\epsilon} d y / I(\epsilon), \quad 0 \leq x \leq 1, \\
I(\epsilon):=\int_{0}^{1} y^{\epsilon}(1-y)^{\epsilon} d y, \quad \epsilon>0 .
\end{gathered}
$$

Diese Funktion hat die folgenden Eigenschaften:

$$
\begin{gathered}
1=b(0) \geq b(x) \geq b(1)=0 \quad \text { für } \quad 0 \leq x \leq 1, \\
b^{\prime}(0)=b^{\prime}(1)=0, \\
b^{\prime}\left(\frac{1}{2}\right)=-1 /\left(4^{\epsilon} I(\epsilon)\right), \\
b^{\prime \prime}(x)<0 \quad \text { für } \quad x \in\left(0, \frac{1}{2}\right), \quad b^{\prime \prime}(x)>0 \quad \text { für } \quad x \in\left(\frac{1}{2}, 1\right), \\
\int_{0}^{1} b(x) d x=\frac{1}{2} .
\end{gathered}
$$

Nun definieren wir für $0<h<R$ die Funktionen $k_{R} \in C_{0}^{1}[0, \infty)$ durch

$$
k_{R}(r)= \begin{cases}1 & \text { für } 0 \leq r \leq R-h_{1}, \\ b\left(\frac{r-R+h_{1}}{h_{1}}\right) & \text { für } R-h_{1}<r \leq R, \\ 0 & \text { für } r>R .\end{cases}
$$

Für $\epsilon \rightarrow 0$ konvergiert $b$ gegen die Funktion $b_{0}: x \mapsto 1-x$. Würden wir in den Definitionen von $k_{R}$, statt $b$ die Funktion $b_{0}$ einsetzten, so wäre $k_{R}$ in den Punkten $R-h 1, R$ nicht differenzierbar.

Mit

$$
K^{b}(R, p):=\sum_{\omega \in Z} k_{R}(\|\omega-p\|), \quad K^{\sharp}(R, p):=\sum_{\omega \in Z} k_{R+h}(\|\omega-p\|)
$$

gilt

$$
K^{b}(R, p) \leq N(R, p) \leq K^{\sharp}(R, p) .
$$

Wir werden nun $K_{1}(R, \cdot)$ und $K_{2}(R, \cdot)$ mit den in Kapitel 2.1 gewonnenen Ergebnissen in Eigenfunktionen des Laplaceoperators zerlegen und so eine obere und untere Abschätzung der Gitterpunktzählfunktion erhalten. Wir benötigen dazu Abschätzungen der Spektralfunktion $g_{k_{R}}$. 
Lemma 2.2.1. Für $\lambda>0$ und $R>0$ gilt

$$
\left|g_{k_{R}}(\lambda)\right|=2 \pi\left|\int_{0}^{R} k_{R}(r) J_{0}(\sqrt{\lambda} r) r d r\right| \leq \frac{2 \pi R^{1 / 2}}{\lambda^{3 / 4}} .
$$

Beweis. Für $G_{\lambda}(r):=\int_{0}^{r} J_{0}(\sqrt{\lambda} x) x d x$ gilt:

$$
\begin{aligned}
\left|G_{\lambda}(r)\right| & =\left|\lambda^{-1}\left[x J_{1}(x)\right]_{0}^{\sqrt{\lambda} r}\right| \\
& =\frac{r}{\sqrt{\lambda}}\left|J_{1}(\sqrt{\lambda} r)\right| \\
& \leq \frac{r^{1 / 2}}{\lambda^{3 / 4}}
\end{aligned}
$$

denn es ist $\frac{d}{d x}\left[x J_{1}(x)\right]=x J_{0}(x)$ (siehe Watson [41] Kapitel $2 \cdot 12$ ), und es gilt $\left|J_{1}(x)\right| \leq$ $x^{-1 / 2}$ für $x>0$. Die letzte Ungleichung für $J_{1}$ folgert man leicht aus den von Watson in [41] Kap. $7 \cdot 3$ gegebenen numerischen Abschätzungen des Restterms in der asymptotischen Entwicklung von $J_{1}$ und aus der Tatsache, daß $\left|J_{1}(x)\right|<1 / \sqrt{2}$ für $x>0$ (siehe Watson [41] Kapitel $2 \cdot 5$ ). Weiter folgt durch partielle Integration

$$
\begin{aligned}
\left|\int_{0}^{R} k_{R}(r) J_{0}(\sqrt{\lambda} r) r d r\right| & =\left|\int_{0}^{R} k_{R}(r) G_{\lambda}^{\prime}(r) d r\right| \\
& =\left|\int_{R-h}^{R} G_{\lambda}(r) k_{R}^{\prime}(r) d r\right| \\
& \leq \int_{R-h}^{R}\left|G_{\lambda}(r)\right|\left|k_{R}^{\prime}(r)\right| d r .
\end{aligned}
$$

Wegen $k_{R}^{\prime}(r) \leq 0$ ist

$$
\int_{R-h}^{R}\left|k_{R}^{\prime}(r)\right| d r=k_{R}(R-h)-k_{R}(R)=1 .
$$

Zusammen folgt die Behauptung.

Lemma 2.2.2. Für $\lambda>0, R>0$ gilt

$$
\left|g_{k_{R}}(\lambda)\right|=2 \pi\left|\int_{0}^{R} k_{R}(r) J_{0}(\sqrt{\lambda} r) r d r\right| \leq \frac{11 \pi R^{1 / 2}}{2 \cdot 4^{\epsilon} I(\epsilon) h \lambda^{5 / 4}}
$$

Wir definieren $e_{\lambda}(r):=J_{0}(\sqrt{\lambda} r)$. Es gilt $\lambda r e_{\lambda}(r)=\frac{d}{d r}\left(-r e_{\lambda}^{\prime}(r)\right)$. Daraus folgt mit zweimaliger partieller Integration und Eigenschaft 2.2.3

$$
\begin{aligned}
\lambda \int_{0}^{R} k_{R}(r) e_{\lambda}(r) r d r & =\int_{0}^{R} k_{R}(r) \frac{d}{d r}\left(-r e_{\lambda}^{\prime}(r)\right) d r \\
& =\int_{R-h}^{R} k_{R}^{\prime}(r) r e_{\lambda}^{\prime}(r) d r \\
& =-\int_{R-h}^{R}\left(r k_{R}^{\prime \prime}(r)+k_{R}^{\prime}(r)\right) e_{\lambda}(r) d r
\end{aligned}
$$


Aus der von Watson in [41] Kapitel $7 \cdot 3$ gegebenen numerischen Abschätzung des Restterms in der Asymptotischen Entwicklung von $J_{0}$ und aus $\left|J_{0}(x)\right|<1$ für $x>0$ (siehe Watson [41] Kap 2.5) folgt, daß $\left|J_{0}(x)\right| \leq x^{-1 / 2}$ für $x>0$ ist. Aus 2.2 .4 folgt $k_{R}^{\prime}(R-h / 2)=-1 /\left(4^{\epsilon} I(\epsilon) h\right)$. Durch partielle Integration und 2.2.5 ergibt sich damit

$$
\begin{aligned}
\left|\int_{0}^{R} k_{R}(r) e_{\lambda}(r) r d r\right| & \leq \lambda^{-5 / 4} \int_{R-h}^{R}\left(r\left|k_{R}^{\prime \prime}(r)\right|-k_{R}^{\prime}(r)\right) r^{-1 / 2} d r \\
& =\lambda^{-5 / 4} \int_{R-h}^{R}\left(\left|k_{R}^{\prime \prime}(r)\right|+2 k_{R}^{\prime \prime}(r)\right) r^{1 / 2} d r \\
& =\lambda^{-5 / 4}\left\{\int_{R-h}^{R-h / 2} k_{R}^{\prime \prime}(r) r^{1 / 2} d r+3 \int_{R-h / 2}^{R} k_{R}^{\prime \prime}(r) r^{1 / 2} d r\right\} \\
& \leq \lambda^{-5 / 4}\left\{\left(R-\frac{h}{2}\right)^{\frac{1}{2}} \int_{R-h}^{R-h / 2} k_{R}^{\prime \prime}(r) d r+3 R^{\frac{1}{2}} \int_{R-h / 2}^{R} k_{R}^{\prime \prime}(r) d r\right\} \\
& =\frac{R^{\frac{1}{2}}}{4^{\epsilon} I(\epsilon) h \lambda^{5 / 4}}\left\{3-\left(1-\frac{h}{2 R}\right)^{\frac{1}{2}}\right\} \\
& \leq \frac{11 R^{\frac{1}{2}}}{4 \cdot 4^{\epsilon} I(\epsilon) h \lambda^{5 / 4}} .
\end{aligned}
$$

Beweis von Theorem A. Aus Lemma 2.2.2 folgt, daß $k_{R}$ die Voraussetzungen von Theorem 2.1.3 erfüllt. Es gilt also

$$
K^{b}(p)=\frac{1}{\operatorname{area}(T)} \sum_{i} g_{k_{R}}\left(\lambda_{i}\right) \phi_{i}(p), \quad K^{\sharp}(p)=\frac{1}{\operatorname{area}(T)} \sum_{i} g_{k_{R+h}}\left(\lambda_{i}\right) \phi_{i}(p)
$$

und die Summen konvergieren absolut. Wir definieren für $s>\lambda_{1}$

$$
\begin{aligned}
R_{1}(p, R, h, s) & :=\sum_{\left\{i: \lambda_{1} \leq \lambda_{i}<s\right\}}\left|g_{k_{R}}\left(\lambda_{i}\right)\right|, \\
R_{2}(p, R, h, s) & :=\sum_{\left\{i: \lambda_{i} \geq s\right\}}\left|g_{k_{R}}\left(\lambda_{i}\right)\right| .
\end{aligned}
$$

Es gilt dann

$$
\begin{gathered}
N(R, p) \geq K^{b}(R, p) \geq \frac{\pi(R-h)^{2}}{\operatorname{area}(T)}-\frac{1}{\operatorname{area}(T)} \sum_{n=1}^{2} R_{n}(p, R, h, s), \\
N(R, p) \leq K^{\sharp}(R, p) \leq \frac{\pi(R+h)^{2}}{\operatorname{area}(T)}+\frac{1}{\operatorname{area}(T)} \sum_{n=1}^{2} R_{n}(p, R+h, h, s) .
\end{gathered}
$$

Wir werden nun die Restterme $R_{1}(p, R, h, s)$ und $R_{2}(p, R, h, s)$ nach oben abschätzen. Für die Abschätzung von $R_{1}$ benutzen wir die Abschätzung von $g_{k_{R}}$ aus Lemma 2.2.1. Diese ist gut in $R$, jedoch nicht geeignet, die Konvergenz der Summe zu erhalten. Für 
die Abschätzung von $R_{2}$ ziehen wir Lemma 2.2.2 heran und erhalten eine konvergente Summe. Der Parameter $s$ wird später so in Abhängigkeit vom Radius $R$ gewählt werden, daß wir die bestmögliche Abschätzung von $K^{b}$ und $K^{\sharp}$ erhalten. Würden wir nur Lemma 2.2.2 benutzen, so bekämen wir in Theorem B lediglich $d(R)=O\left(R^{\frac{3}{4}}\right)$. In unserer Rechnung benötigen wir das folgende einfache, unbestimmte Integral

$$
\int x^{\mu}(\sqrt{x}+d)^{2} d x=x^{\mu+1}\left(\frac{x}{2+\mu}+\frac{2 d x^{\frac{1}{2}}}{\frac{3}{2}+\mu}+\frac{d^{2}}{\mu+1}\right), \quad \text { für } \quad \mu \notin\{-2,-3 / 2,-1\} .
$$

Mit Lemma 2.2.1 und Proposition 2.1.2 folgt

$$
\begin{aligned}
R_{1}(p, R, h, s) & =\int_{\lambda_{1}}^{s}\left|g_{k_{R}}(x)\right| d N_{\lambda}(x) \\
& \leq 2 \pi R^{\frac{1}{2}} \int_{\lambda_{1}}^{s} x^{-\frac{3}{4}} d N_{\lambda}(x) \\
& =2 \pi R^{\frac{1}{2}}\left\{\left[x^{-\frac{3}{4}} N_{\lambda}(x)\right]_{\lambda_{1}}^{s}+\frac{3}{4} \int_{\lambda_{1}}^{s} x^{-\frac{7}{4}} N_{\lambda}(x) d x\right\} \\
& \leq \frac{1}{2} \operatorname{area}(T) R^{\frac{1}{2}}\left\{s^{-\frac{3}{4}}(\sqrt{s}+d)^{2}+3 s^{\frac{1}{4}}+6 d \lambda_{1}^{-\frac{1}{4}}+d^{2} \lambda_{1}^{-\frac{3}{4}}\right\} \\
& \leq 2 \operatorname{area}(T) R^{\frac{1}{2}}\left(s^{\frac{1}{4}}+2 d \lambda_{1}^{-\frac{1}{4}}+\frac{1}{2} d^{2} \lambda_{1}^{-\frac{3}{4}}\right) .
\end{aligned}
$$

Wegen Lemma 2.2.2 und Proposition 2.1.2 gilt

$$
\begin{aligned}
R_{2}(p, R, h, s) & =\int_{s}^{\infty}\left|g_{k_{R}}(x)\right| d N_{\lambda}(x) \\
& \leq \frac{11 \pi R^{\frac{1}{2}}}{2 \cdot 4^{\epsilon} I(\epsilon) h} \int_{s}^{\infty} x^{-\frac{5}{4}} d N(x) \\
& \leq \frac{11 \pi R^{\frac{1}{2}}}{2 \cdot 4^{\epsilon} I(\epsilon) h} \cdot \frac{5}{4} \int_{s}^{\infty} x^{-\frac{9}{4}} N(x) d x \\
& \leq \frac{55 \operatorname{area}(T) R^{\frac{1}{2}}}{8 \cdot 4^{\epsilon} I(\epsilon) h}\left[-4 x^{-\frac{1}{4}}-\frac{8}{3} d x^{-\frac{3}{4}}-\frac{4}{5} d^{2} x^{-\frac{5}{4}}\right]_{s}^{\infty} \\
& \leq \frac{55 \operatorname{area}(T)}{2 \cdot 4^{\epsilon} I(\epsilon)} \cdot \frac{R^{\frac{1}{2}}}{h}\left(s^{-\frac{1}{4}}+\frac{2}{3} d s^{-\frac{3}{4}}+\frac{1}{5} d^{2} s^{-\frac{5}{4}}\right) .
\end{aligned}
$$

Der Einfachheit halber setzen wir

$$
\begin{gathered}
A:=\frac{2 \pi}{\operatorname{area}(T)}, \quad B:=\frac{55}{2 \cdot 4^{\epsilon} I(\epsilon)}, \\
C:=2 d \lambda_{1}^{-\frac{1}{4}}+\frac{1}{2} d^{2} \lambda_{1}^{-\frac{3}{4}} .
\end{gathered}
$$


Es sei $s>\max \left(1, \lambda_{1}\right)$. Dann folgt aus 2.2.8, 2.2.10 und 2.2.11, daß

$$
\begin{gathered}
N(R, p) \geq K^{b}(R, p) \geq \frac{\pi}{\operatorname{area}(T)} R^{2}-d_{1}(R, h, s) \quad \text { mit } \\
d_{1}(R, h, s):=A R h+R^{\frac{1}{2}}\left(2 s^{\frac{1}{4}}+2 C+B h^{-1} s^{-\frac{1}{4}}+B h^{-1} s^{-\frac{1}{2}}\left(\frac{2}{3} d s^{-\frac{1}{4}}+\frac{1}{5} d^{2} s^{-\frac{3}{4}}\right)\right) .
\end{gathered}
$$

Da $s>1$ ist, gilt

$$
\begin{gathered}
d_{1}(R, h, s) \leq \widetilde{d}_{1}(R, h, s):=A R h+R^{\frac{1}{2}}\left(2 s^{\frac{1}{4}}+2 C+B h^{-1} s^{-\frac{1}{4}}+D B h^{-1} s^{-\frac{1}{2}}\right), \\
D:=\frac{2}{3} d+\frac{1}{5} d^{2} .
\end{gathered}
$$

Unter Vernachlässigung des letzten Terms hat $\widetilde{d}_{1}(R, \cdot, \cdot)$ ein Minimum bei

$$
h^{3}=\frac{2 B}{A^{2}} R^{-1}, \quad s^{\frac{3}{4}}=\frac{A B}{4} R^{\frac{1}{2}} .
$$

Mit dieser Wahl für $h$ und $s$ gilt für $R>\left(\frac{4}{A B}\right)^{2} \max \left\{\lambda_{1}^{\frac{3}{2}}, 1\right\}$, daß

$$
\widetilde{d}_{1}(R, h, s)=3(2 A B)^{\frac{1}{3}} R^{\frac{2}{3}}+2(C+D) R^{\frac{1}{2}} .
$$

Läßt man noch $\epsilon$ gegen Null gehen, so folgt für $R \geq \frac{\operatorname{area}^{2}(T)}{1875} \max \left\{\lambda_{1}^{\frac{3}{2}}, 1\right\}$,

$$
d_{1}\left(R, h_{1}, s\right) \leq 21.1 \cdot \operatorname{area}^{\frac{1}{3}}(T) R^{\frac{2}{3}}+a_{1} R^{\frac{1}{2}}
$$

mit

$$
a_{1}:=d^{2}\left(\lambda_{1}^{-\frac{3}{4}}+\frac{2}{5}\right)+4 d\left(\lambda_{1}^{-\frac{1}{4}}+\frac{1}{3}\right) .
$$

Aus 2.2.9, 2.2.10 und 2.2.11 folgt

$$
\begin{aligned}
N(R, p) \geq & K^{\sharp}(R, p) \geq \frac{\pi}{\operatorname{area}(T)} R^{2}+d_{2}(R, h, s) \text { mit } \\
d_{2}(R, h, s):= & A R h+A h^{2}+(R+h)^{\frac{1}{2}} . \\
& \cdot\left(2 s^{\frac{1}{4}}+2 C+B h_{1}^{-1} s^{-\frac{1}{4}}+B h^{-1} s^{-\frac{1}{2}}\left(\frac{2}{3} d s^{-\frac{1}{4}}+\frac{1}{5} d^{2} s^{-\frac{3}{4}}\right)\right) .
\end{aligned}
$$

Für $s \geq 1$ und mit $h<R$ gilt

$d_{2}(R, h, s) \leq \widetilde{d}_{2}(R, h, s):=A R h+A h^{2}+\sqrt{2} R^{\frac{1}{2}}\left(2 s^{\frac{1}{4}}+2 C+B h^{-1} s^{-\frac{1}{4}}+D B h^{-1} s^{-\frac{1}{2}}\right)$.

Mit der Wahl

$$
h^{3}=\frac{2 B}{A^{2}} R^{-1}, \quad s^{\frac{3}{4}}=\frac{A B}{4} R^{\frac{1}{2}},
$$


und es folgt

$$
\widetilde{d}_{2}(R, h, s) \leq(2+\sqrt{2})(2 A B)^{\frac{1}{3}} R^{\frac{2}{3}}+\frac{(2 B)^{\frac{2}{3}}}{A^{\frac{1}{3}}} R^{-\frac{2}{3}}+2 \sqrt{2}(C+D) R^{\frac{1}{2}} .
$$

Für $R \geq \frac{55}{\sqrt{2 \pi}}(\operatorname{area}(T))^{2}$ gilt

$$
\frac{(2 B)^{\frac{2}{3}}}{A^{\frac{1}{3}}} R^{-\frac{2}{3}} \leq \operatorname{area}^{-1}(T)
$$

Für $R \geq(\operatorname{area}(T))^{2} \cdot \max \left\{\frac{\lambda_{1}^{\frac{3}{2}}}{1875}, 22\right\}$ gilt dann

$$
d_{2}\left(R, h_{1}, s\right) \leq 24.1 \cdot \operatorname{area}^{\frac{1}{3}}(T) R^{\frac{2}{3}}+\sqrt{2} a_{1} R^{\frac{1}{2}}+\operatorname{area}^{-1}(T) .
$$

Damit ist Theorem A bewiesen. 


\section{Das Gitterpunktproblem in der hyperbolischen Ebene}

Das folgende Kapitel untersucht die Asymptotik der Gitterpunktzählfunktion Fuchsscher Gruppen. Vorbereitend dazu wird in Abschnitt 3.1 die Spektraltheorie Fuchsscher Gruppen diskutiert. Die wichtigsten Resultate sind in Proposition 3.1.3 und Theorem 3.1.5 formuliert. Im Abschnitt 3.1.2 werden die für uns wichtigen Eigenschaften des Spektrums geometrisch endlicher Fuchsscher Gruppen erläutert. Abschnitt 3.2 enthält den Beweis des Theorems B, und Abschnitt 3.3 ist den numerischen Abschätzungen der Zählfunktion gewidmet, welche in Theorem C und D formuliert sind. Schließlich werden Theorem C und D im Abschnitt 3.4 angewendet, um einerseits in Korollar B für eine Fuchssche Gruppe einen Test auf einen (neben Null) weiteren diskreten Eigenwert zu erhalten und um andererseits in Korollar C den Konvergenzexponenten sowie die erste Eigenfunktion einer Fuchsschen Gruppe vermittels ihrer Gitterpunktzählfunktion zu approximieren. Ist der Wert der Zählfunktion für zwei Radien bekannt, so liefert Korollar C obere und untere Schranken für beide Werte.

\subsection{Die Spektraltheorie Fuchsscher Gruppen}

In diesem Kapitel sollen die Grundlagen der Spektraltheorie des Laplace-Beltrami Operators auf Riemannschen Flächen behandelt werden. Wir orientieren uns dabei bis zu einem bestimmten Punkt an Patterson [29].

Jede Riemannsche Fläche läßt sich als Quotient $D / G$ darstellen, wobei $D \subset \mathbb{C}$ die Einheitskreisscheibe und $G$ eine beliebige Fuchsche Gruppe bezeichnet. Der Laplace Operator $\Delta$ auf $D$ ist durch

$$
\Delta=\frac{1}{4}\left(1-|z|^{2}\right) \frac{\partial}{\partial z} \frac{\partial}{\partial \bar{z}}
$$

definiert und induziert einen Laplace Operator auf $D / G$ mit einem Definitionsbereich, der dicht in $\mathfrak{H}=L^{2}(D / G)$ liegt. Dieser Operator hat eine selbstadjungierte Fortsetzung (siehe hierzu [35] I, S. 305ff.). Nach dem Spektraltheorem von v. Neumann gibt es ein Maß $\mu$ mit Träger im Spektrum von $-\widetilde{\Delta}$ und eine unitäre Abbildung $T$ von $\mathfrak{H}$ auf das direkte Integral

$$
\int \mathfrak{H}_{\lambda} d \mu(\lambda)
$$

mit der Eigenschaft, daß

$$
T(-\widetilde{\Delta}) T^{-1} u=\lambda u
$$


Die Funktion $J(\lambda):=\operatorname{dim} \mathfrak{H}_{\lambda}$ ist bezüglich $\mu$ meßbar. Das Spektrum von $(-\widetilde{\Delta})$ liegt in $[0, \infty)\left([7]\right.$, II, S. 104). Die Resolvente $(-\Delta-\lambda I)^{-1}$ ist für $\lambda \notin[0, \infty)$ ein Integraloperator vom Carlemantyp, d.h. ist $r(z, w, \lambda)$ der Kern der Resolvente, dann ist

$$
b(z):=\int|r(z, w, \lambda)|^{2} d \sigma(w)<\infty
$$

eine stetige Funktion ([7] I, S. 323). Nach Maurin [21] Kapitel XVII $\S 8$ und $\S 9$ gilt dann sogar:

Theorem 3.1.1. Für eine Fuchssche Gruppe $G$ gibt es ein $M a ß \mu$ auf $\mathbb{R}$ mit Träger in $[0, \infty)$ und eine unitäre Abbildung $T$ von $L^{2}(D / G)$ auf das direkte Integral

$$
\int \mathfrak{H}_{\lambda} d \mu(\lambda)
$$

Die Funktion $J(\lambda):=\operatorname{dim} \mathfrak{H}_{\lambda}$ ist bezüglich $\mu$ meßbar, und es gibt Funktionen $e(\lambda, z)=$ $\left(e_{1}(\lambda, z), \ldots, e_{J(\lambda)}(\lambda, z)\right)$, wobei $e_{j}(\cdot, z)$ meßbar ist und $e_{j}(\lambda, \cdot) \in C^{\infty}(D / G)$, sodaß

$$
-\Delta e_{j}(\lambda, z)=\lambda e_{j}(\lambda, z),
$$

und für jede Funktion $f \in L^{2}(D / G)$ mit

$$
\int|f(z)| b(z) d \sigma(z)<\infty
$$

für $\mu$-fast alle $\lambda$ gilt

$$
(T f)(\lambda)=\int_{D / G} f(z) \overline{e(\lambda, z)} d \sigma(z) .
$$

Bemerkung. Die Funktionen $e(\lambda, \cdot)$ können für eine $\mu$-Nullmenge identisch verschwinden.

Im Folgenden sollen die Funktionen $e(\lambda, z)$ immer für die aus Theorem 3.1.1 stehen. Es ist zu beachten, daß diese vektorwertig sind (evtl. auch unendlichdimensional). Operationen mit ihnen beziehen sich immer auf den zugrundeliegenden Vektorraum. Insbesondere bedeutet die Multiplikation zweier Funktionen das Skalarprodukt ihere Werte.

\subsubsection{Die Spektralzerlegung von Punktpaarinvarianten}

Wir wollen Theorem 3.1.1 auf Integraloperatoren, die durch Punktpaarinvarianten definiert sind, anwenden. Punktpaarinvarianten sind Funktionen $k(z, w): D \times D \mapsto \mathbb{R}$ mit der Eigenschaft, daß

$$
k(z, w)=k(g(z), g(w))
$$

für alle $g \in \operatorname{Con}(1)$. Ist $k(z, w)$ eine Punktpaarinvariante, so gibt es eine Funktion $k(x)$ mit

$$
k(z, w)=k(\cosh d(z, w))
$$


(siehe [29]). Wir betrachten den $G$-invarianten Kern

$$
K(z, w):=\sum_{g \in G} k(z, g(w)) .
$$

Es habe nun $k$ einen kompakten Träger. Wir wollen $K(z, w)$ als Funktion von $z$ auffassen und $[T K(\cdot, w)](\lambda)$ berechnen. Wir wählen dazu $w=0$ und gehen zu Polarkoordinaten $\rho=d(0, z), \vartheta=\arg z$ über. Es ist dann

$$
d s^{2}=d \rho^{2}+\sinh ^{2} \rho d \vartheta^{2},
$$

und für das Flächenelement $d \sigma$ und den Laplaceoperator $\Delta$ erhält man

$$
\begin{gathered}
d \sigma(z(\rho, \vartheta))=\sinh \rho d \rho d \vartheta \\
\Delta=\frac{\partial^{2}}{\partial \rho^{2}}+\operatorname{coth} \rho \frac{\partial}{\partial \rho}+\frac{1}{\sinh ^{2} \rho} \frac{\partial^{2}}{\partial \vartheta^{2}}
\end{gathered}
$$

Es ist

$$
\begin{aligned}
\int_{D / G} K(z, w) \overline{e_{j}(\lambda, z)} d \sigma(z) & =\int_{D} k(z, w) \overline{e_{j}(\lambda, z)} d \sigma(z) \\
& =\int_{0}^{\infty} k(\cosh \rho) \sinh \rho \int_{0}^{2 \pi} \overline{e_{j}(\lambda, z(\rho, \vartheta))} d \vartheta d \rho .
\end{aligned}
$$

Nun soll die Funktion

$$
H_{\lambda, j}(\cosh \rho):=\int_{0}^{2 \pi} e_{j}(\lambda, z(\rho, \vartheta)) d \vartheta
$$

berechnet werden. Es gilt $H_{\lambda, j} \in C^{2}(1,+\infty)$. Da $e_{j}(\lambda, z)$ eine Eigenfunktion von $(-\Delta)$ zum Eigenwert $\lambda$ ist, gilt

$$
\frac{\partial^{2}}{\partial \rho^{2}} e_{j}(\lambda, z)+\operatorname{coth} \rho \frac{\partial}{\partial \rho} e_{j}(\lambda, z)+\frac{1}{\sinh ^{2} \rho} \frac{\partial^{2}}{\partial \vartheta^{2}} e_{j}(\lambda, z)+\lambda e_{j}(\lambda, z)=0 .
$$

Durch Integration über $\vartheta$ ergibt sich

$$
\frac{\partial^{2}}{\partial \rho^{2}} \int_{0}^{2 \pi} e_{j}(\lambda, z) d \vartheta+\operatorname{coth} \rho \frac{\partial}{\partial \rho} \int_{0}^{2 \pi} e_{j}(\lambda, z) d \vartheta+\lambda \int_{0}^{2 \pi} e_{j}(\lambda, z) d \vartheta=0,
$$

und mit der Variablentransformation $t=\cosh \rho$ erhält man

$$
\left(t^{2}-1\right) H_{\lambda, j}^{\prime \prime}(t)+2 t H_{\lambda, j}^{\prime}(t)+\lambda H_{\lambda, j}(t)=0
$$

für $t>1$. Außerdem gilt

$$
\lim _{t \searrow 1} H_{\lambda, j}(t)=2 \pi e_{j}(\lambda, w)
$$


Die Differentialgleichung (3.1.1) ist die sogenannte Legendre-Differentialgleichung und hat nach Lemma A.0.2 im Appendix wegen (3.1.2) genau eine Lösung

$$
H_{\lambda, j}(t)=2 \pi e_{j}(\lambda, w) F_{\lambda}(t)
$$

wobei $F_{\lambda}$ die im Appendix definierte Legendre-Funktion mit $F_{\lambda}(1)=1$ ist. Mit der Definition

$$
h_{k}(\lambda):=2 \pi \int_{1}^{\infty} F_{\lambda}(t) k(t) d t
$$

erhalten wir also

$$
\int_{D / G} K(z, w) \overline{e_{j}(\lambda, z)} d \sigma(z)=h_{k}(\lambda) \overline{e_{j}(\lambda, w)}
$$

Wir wollen nun

$$
\int_{\lambda<X} e^{2}(\lambda, p) d \mu(\lambda)
$$

als Funktion von $X$ nach oben abschätzen und verallgemeinern dazu den Beweis von Huber in [14] für cokompakte Fuchssche Gruppen auf beliebige Fuchssche Gruppen. Es sei nun $\pi: D \rightarrow D / G$ die kanonische Projektion, $p \in D / G$ und $\widetilde{p} \in D$ mit $\pi(\widetilde{p})=p$. Die Kreisscheibe mit hyperbolischem Radius $r$ um $x \in D$ sei mit $B_{r}(x)$ bezeichnet. Der Injektivitätsradius $\operatorname{inj}(p)$ eines Punktes $p \in D / G$ ist durch

$$
\operatorname{inj}(p):=\sup \left\{r \in \mathbb{R}^{+}:\left.\pi\right|_{B_{r}(\widetilde{p})} \text { ist injektiv }\right\}
$$

definiert.

Lemma 3.1.2. Es sei $G$ eine beliebige Fuchssche Gruppe, $p \in D / G$ und $r_{p}:=\operatorname{inj}(p)$ der Injektivitätsradius von $p$. Die Funktion $f:\left[0, \cosh \left(r_{p}\right)\right] \rightarrow \mathbb{C}$ sei quadratintegrabel. Dann ist

$$
\frac{1}{2 \pi} \int_{1}^{\cosh r_{p}} f^{2}(t) d t=\int e^{2}(\lambda, p)\left(\int_{1}^{\cosh r_{p}} f(t) F_{\lambda}(t) d t\right)^{2} d \mu(\lambda) .
$$

Beweis. Es sei

$$
k(z, w):=\left\{\begin{array}{cl}
f(\cosh d(z, w)) & \text { für } d(z, w)<r_{p}, \\
0 & \text { sonst }
\end{array}\right.
$$

und $K(z, w):=\sum_{g \in G} k(z, g(w))$. Dann ist einerseits

$$
\begin{aligned}
\|K(\cdot, p)\|^{2} & =\int k^{2}(z, p) d \sigma(z)=2 \pi \int_{0}^{r_{p}} f^{2}(\cosh \rho) \sinh \rho d \rho \\
& =2 \pi \int_{1}^{\cosh r_{p}} f^{2}(t) d t
\end{aligned}
$$


und andererseits gilt nach Theorem 3.1.1 und Gleichung (3.1.4) aber

$$
\begin{aligned}
\|K(\cdot, p)\|^{2} & =\|T K(\cdot, p)\|^{2}=\int\|[T K(\cdot, p)](\lambda)\|^{2} d \mu(\lambda) \\
& =\int h_{k}^{2}(\lambda) e^{2}(\lambda, p) d \mu(\lambda) \\
& =(2 \pi)^{2} \int e^{2}(\lambda, p)\left(\int_{1}^{\cosh r_{p}} f(t) F_{\lambda}(t) d t\right)^{2} d \mu(\lambda) .
\end{aligned}
$$

Proposition 3.1.3. Es sei $G$ eine beliebige Fuchssche Gruppe, $p \in D / G$ und $r_{p}:=$ $\operatorname{inj}(p)$ der Injektivitätsradius von $p$. Dann ist für $X \geq \sinh ^{-2}\left(r_{p} / 2\right)$,

$$
\int_{\lambda<X} e^{2}(\lambda, p) d \mu(\lambda) \leq \frac{3}{4 \pi} X
$$

Beweis. Es sei nun $X \geq \sinh ^{-2}\left(r_{p} / 2\right)$, was äquivalent zu $1+2 / X \leq \cosh r_{p}$ ist. Wir definieren die Funktion

$$
f(t):= \begin{cases}F_{X}(t) & \text { für } 1 \leq t<1+2 / X, \\ 0 & \text { für } 1+2 / X \leq t \leq \cosh r_{p} .\end{cases}
$$

Aus Lemma 3.1.2 folgt

$$
\begin{aligned}
\frac{1}{2 \pi} \int_{1}^{1+2 / X} F_{X}^{2}(t) d t & =\int e^{2}(\lambda, p)\left(\int_{1}^{1+2 / X} F_{X}(t) F_{\lambda}(t) d t\right)^{2} d \mu(\lambda) \\
& \geq \int_{\lambda<X} e^{2}(\lambda, p)\left(\int_{1}^{1+2 / X} F_{X}(t) F_{\lambda}(t) d t\right)^{2} d \mu(\lambda) .
\end{aligned}
$$

Aus Lemma A.0.4 und Lemma A.0.5 folgt

$$
F_{\lambda}(t) \geq F_{X}(t) \geq 0 \quad \text { in } \quad[0,1+2 / X] \quad \text { für } \quad \lambda<X,
$$

und es gilt also

$$
\int_{1}^{1+2 / X} F_{X}(t) F_{\lambda}(t) d t \geq \int_{1}^{1+2 / X} F_{X}^{2}(t) d t \quad \text { für } \quad \lambda<X .
$$

Zusammen ergibt sich

$$
\int_{1}^{1+2 / X} F_{X}^{2}(t) d t \int_{\lambda<X} e^{2}(\lambda, p) d \mu(\lambda) \leq(2 \pi)^{-1} .
$$

Schließlich ist wegen Lemma A.0.4

$$
\int_{1}^{1+2 / X} F_{X}^{2}(t) d t \geq \int_{1}^{1+2 / X}\left(1-\frac{X}{2}(t-1)\right)^{2} d t=\frac{2}{3 X},
$$

und mit (3.1.6) folgt die Behauptung. 
Aus der Cauchy-Schwarz-Ungleichung folgt:

Korollar 3.1.4. Es sei $G$ eine beliebige Fuchssche Gruppe und $r:=\min \{\operatorname{inj}(p), \operatorname{inj}(q)\}$. Dann ist für $X \geq \sinh ^{-2}(r / 2)$

$$
\int_{\lambda<X}|e(\lambda, p) \overline{e(\lambda, q)}| d \mu(\lambda) \leq \frac{3}{4 \pi} X .
$$

Mit Hilfe dieses Korollars beweisen wir nun

Theorem 3.1.5. Es sei $G$ eine beliebige Fuchssche Gruppe und $k$ eine stetige Punktpaarinvariante mit kompaktem Träger. Es sei $K(p, q)=\sum_{g \in G} k(p, g(q))$ und

$$
h_{k}(\lambda)=2 \pi \int_{1}^{\infty} F_{\lambda}(t) k(t) d t .
$$

Ist nun $h_{k}(\lambda)=O\left((1+\lambda)^{-\beta}\right)$ für ein $\beta>1$, so gilt, falls $p$ und $q$ keine Verzweigungspunkte sind, daß

$$
K(p, q)=\int e(\lambda, p) \overline{e(\lambda, q)} h_{k}(\lambda) d \mu(\lambda) .
$$

Das Integral konvergiert absolut.

Beweis. Es sei $\mathfrak{K} \subset D / G$ ein beliebiges Kompaktum, das keine Verzweigungspunkte enthält. Dann ist $r:=\inf \{\operatorname{inj}(p): p \in \mathfrak{K}\}>0$ und nach Korollar 3.1.4 gilt

$$
\int_{\lambda<X}|e(\lambda, p) \overline{e(\lambda, q)}| d \mu(\lambda) \leq \frac{3}{4 \pi} X
$$

für $X \geq \sinh ^{-2}(r / 2)$. Es folgt, daß

$$
\int_{\lambda>X}|e(\lambda, p) \overline{e(\lambda, q)}|\left|h_{k}(\lambda)\right| d \mu(\lambda)<C X^{1-\beta}
$$

mit einer Konstanten $C$ unabhängig von $p, q$ und $X$. Das Integral aus 3.1.7 konvergiert also als Funktion auf $\mathfrak{K} \times \mathfrak{K}$ absolut und gleichmäßig. Die Behauptung folgt nun aus der $L^{2}$-Konvergenz gegen $K(\cdot, \cdot)$ und der Stetigkeit von $K(\cdot, \cdot)$.

Patterson beweist die obige Aussage in [29] ohne die Einschränkung, daß $p, q$ keine Verzweigungspunkte seien dürfen und für eine größere Klasse von Punktpaarinvarianten $k$.

\subsubsection{Das $\mathrm{L}^{2}$-Spektrum Fuchsscher Gruppen}

Um im folgenden Kapitel die Gitterpunktzählfunktion abschätzen zu können, benötigen wir Information über das Spektralmaß der zugrunde liegenden Fuchsschen Gruppe im Intervall [0,1/4]. Für geometrisch endliche Gruppen haben Lax und Phillips in [19] bewiesen, daß das Spektrum in $[0,1 / 4]$ aus nur endlich vielen diskreten Eigenwerten besteht. Für eine Fuchssche Gruppe $G$ mit area $(D / G)<\infty$ ist der kleinste Eigenwert 
$\lambda_{1}=0$. Wenn area $(D / G)=\infty$ ist, ist der kleinste Eigenwert $\lambda_{1}>0$. Die diskreten Eigenwerte sollen im weiteren mit $\lambda_{1}, \lambda_{2}, \ldots, \lambda_{n}$ bezeichnet werden. Die zugehörigen normierten Eigenfunktionen seien mit $\phi_{1}, \phi_{2}, \ldots, \phi_{n}$ bezeichnet. Sie können alle reellwertig gewählt werden. Wir werden die Existenz mindestens eines diskreten Eigenwertes benötigen. Für geometrisch endliche Fuchssche Gruppen mit Konvergenzexponenten $\delta>1 / 2$ gibt es stets einen diskreten Eigenwert. Die Eigenfunktion zum kleinsten Eigenwert $\lambda_{1}=\delta(1-\delta)$ kann in diesem Fall mit Hilfe des Patterson Maßes konstruiert werden (siehe Patterson [28], [30]). Als Folgerung ergibt sich insbesondere, daß die Eigenfunktion zum kleinsten Eigenwert $\lambda_{1}$ stets echt positiv ist. Da die übrigen Eigenfunktionen orthogonal zu $\phi_{1}$ sind, müssen sie sowohl negative als auch positive Werte annehmen.

\subsection{Die Asymptotik der Gitterpunktzählfunktion}

Für eine Fuchssche Gruppe $G$ betrachten wir nun die in Abschnitt 1.1 definierte Gitterpunktzählfunktion $N(a ; z, w)$. Um obere und untere Abschätzungen für diese Funktion zu finden, gehen wir analog zum euklidischen Fall vor. Die Spektraltheorie für geometrisch endliche Fuchssche Gruppen unterscheidet sich jedoch deutlich von der für den euklidischen Torus. Zum einen sind die zu den von uns betrachteten Fuchsschen Gruppen gehörigen Flächen nicht notwendig kompakt, und es gibt deshalb einen kontinuierlichen Anteil am Spektrum, zum anderen wird der führende Term der Zählfunktion bei geometrisch endlichen Fuchsschen Gruppen von allen diskreten Eigenwerten kleiner als 1/4 bestimmt, während er im euklidischen Fall vom Eigenwert $\lambda_{0}=0$ stammt. Wir werden in diesem Kapitel das folgende Theorem beweisen.

Theorem B. Es sei $G$ eine geometrisch endliche torsionsfreie Fuchssche Gruppe mit Konvergenzexponenten $\delta>1 / 2$, diskreten Eigenwerten $\lambda_{1}, \lambda_{2}, \ldots, \lambda_{n} \in[0,1 / 4)$ und zugehörigen normierten reellen Eigenfunktionen $\phi_{1}, \phi_{2}, \ldots, \phi_{n} \in L^{2}(D / G)$. Es sei $s_{k}:=$ $\frac{1}{2}+\sqrt{\frac{1}{4}-\lambda_{k}}$ und also $\delta=s_{1}$. Dann gilt

$$
N(a ; z, w)=\sum_{1 \geq s_{k}>\frac{1}{3}(1+\delta)} 2 \sqrt{\pi} \frac{\Gamma\left(s_{k}-1 / 2\right)}{\Gamma\left(1+s_{k}\right)} \phi_{k}(z) \phi_{k}(w) a^{s_{k}}+O\left(a^{\frac{1}{3}(1+\delta)}\right) .
$$

Für $\delta=1$ erhält man Selbergs Ergebnis für cofinite Fuchssche Gruppen. Lax und Phillips zeigen in [19] eine asymptotische Abschätzung -wie die in Theorem B- mit anderen Methoden. Ihr Fehlerterm ist von der Ordnung $O\left((\log a)^{5 / 6} a^{1 / 3(1+\delta)}\right)$. Theorem B liefert also ein etwas besseres Ergebnis.

Es sei $G$ eine Fuchssche Gruppe und

$$
k_{0}(r):=\left\{\begin{array}{lll}
1 & \text { für } 0 \leq r \leq a \\
0 & \text { für } r>a
\end{array}\right.
$$

Dann gilt für die Gitterpunktzählfunktion, daß

$$
N(a ; z, w)=\sum_{g \in G} k_{0}(\cosh d(g(z), w)) .
$$


Um absolute Konvergenz in der Spektralentwickung zu bekommen, müssen wir $k_{0}$ wie im euklidischen Fall durch eine "glattere" Funktion approximieren. Wir definieren hierzu wie in Abschnitt 1.4

$$
b(x):=2 x^{3}-3 x^{2}+1
$$

und damit für $h>0$ die Funktion $k_{a} \in C_{0}^{1}[0, \infty)$ durch

$$
k_{a}(r)= \begin{cases}1 & \text { für } 0 \leq r \leq a-h, \\ b\left(\frac{r-a+h}{h}\right) & \text { für } a-h<r \leq a, \\ 0 & \text { für } r>a .\end{cases}
$$

In Abschnitt 1.4 findet man eine Abbildung, die $k_{a}$ (mit $R$ statt $a$ ) darstellt. Huber definiert in [15] eine Familie von Funktionen, in der unsere Funktion $k_{a}$ enthalten ist.

Folgende Eigenschaften von $k_{a}$ werden später wichtig sein

$$
\begin{gathered}
k_{a}^{\prime}(a-h)=0, \quad k_{a}^{\prime}(a)=0 \\
\int_{a}^{a-h}\left|k_{a}^{\prime \prime}(x)\right| d x=h^{-1} \int_{0}^{1}\left|b^{\prime \prime}(x)\right| d x=3 h^{-1} .
\end{gathered}
$$

Mit

$$
\begin{aligned}
K_{a}^{b}(z, w) & :=\sum_{g \in G} k_{a}(\cosh d(g(z), w)), \\
K_{a}^{\sharp}(z, w) & :=\sum_{g \in G} k_{a+h}(\cosh d(g(z), w))
\end{aligned}
$$

gilt

$$
K_{a}^{b}(z, w) \leq N(a ; z, w) \leq K_{a}^{\sharp}(z, w) .
$$

Wir wollen nun die Spektralfunktion $h_{k_{a}}$ abschätzen. Die Eigenwerte kleiner als 1/4 geben uns später den führenden Term in der Asymptotik der Gitterpunktzählfunktion.

Lemma 3.2.1. Für $0<\lambda<1 / 4, a>1, h<a$ und mit $s(\lambda):=\frac{1}{2}+\sqrt{\frac{1}{4}-\lambda}$ gilt

$$
h_{k_{a}}(\lambda)=2 \sqrt{\pi} \frac{\Gamma(s(\lambda)-1 / 2)}{\Gamma(1+s(\lambda))} a^{s(\lambda)}+O\left(h a^{s(\lambda)-1}\right)+O\left(a^{1 / 2}\right)
$$

und genauso

$$
h_{k_{a+h}}(\lambda)=2 \sqrt{\pi} \frac{\Gamma(s(\lambda)-1 / 2)}{\Gamma(1+s(\lambda))} a^{s(\lambda)}+O\left(h a^{s(\lambda)-1}\right)+O\left(a^{1 / 2}\right) .
$$

Beweis. Aus Korollar A.1.2 folgt

$$
\begin{aligned}
& h_{k_{a}}(\lambda) \leq 2 \sqrt{\pi} \frac{\Gamma(s(\lambda)-1 / 2)}{\Gamma(1+s(\lambda))} a^{s(\lambda)}+O\left(a^{-\frac{1}{2}}\right) \\
& h_{k_{a}}(\lambda) \geq 2 \sqrt{\pi} \frac{\Gamma(s(\lambda)-1 / 2)}{\Gamma(1+s(\lambda))}(a-h)^{s(\lambda)}+O\left((a-h)^{1 / 2}\right) .
\end{aligned}
$$


Mit $a^{s} \geq(a-h)^{s}=a^{s}-s \int_{a-h}^{a} x^{s-1} d x \geq a^{s}-s h a^{s-1}$ für $0 \leq s \leq 1$ folgt nun (3.2.4). Und aus (3.2.4) folgt mit $a^{s} \leq(a+h)^{s}=a^{s}+s \int_{a}^{a+h} x^{s-1} d x \leq a^{s}+s h(a+h)^{s-1}$ für $0 \leq s<1$ und $h<a$ auch (3.2.5).

Der Wert $\lambda=1 / 4$ bedarf einer besonderen Betrachtung.

Lemma 3.2.2. Mit $a=\cosh \rho, a>1$ und $\lambda \geq 1 / 4$ gilt

$$
\left|h_{k_{a}}(\lambda)\right| \leq h_{k_{a}}(1 / 4)=O\left(\rho a^{1 / 2}\right) \text {. }
$$

Beweis. Aus den Integraldarstellungen (A.0.2) und (A.0.3) für $F_{\lambda}$ folgt $\left|F_{\lambda}(t)\right| \leq F_{1 / 4}(t)$ für $t>1, \lambda \geq 1 / 4$. Damit folgt

$$
\begin{aligned}
\left|h_{k_{a}}(\lambda)\right| & =2 \pi\left|\int_{1}^{a} F_{\lambda}(t) k_{a}(t) d t\right| \\
& \leq 2 \pi \int_{1}^{a}\left|F_{\lambda}(t)\right| k_{a}(t) d t \\
& \leq 2 \pi \int_{1}^{a} F_{1 / 4}(t) k_{a}(t) d t=h_{k_{a}}(1 / 4) \\
& \leq 2 \pi \int_{1}^{a} F_{1 / 4}(t) d t .
\end{aligned}
$$

Die Behauptung folgt nun aus Lemma A.2.1.

Die folgenden beiden Lemmata geben Abschätzungen für die Spektralfunktion $h_{k_{a}}$ für große $\lambda$. Huber beweist solche Abschätzungen in [15] mit expliziten Konstanten. In der Form, wie wir sie hier angeben, müssen wir $\lambda$ von $1 / 4$ weg beschränken. Der Einfachheit halber wählen wir als Voraussetzung $\lambda>1$.

Lemma 3.2.3. Für $\lambda>1$ gilt

$$
\left|h_{k_{a}}(\lambda)\right|=O\left(a^{1 / 2} \lambda^{-3 / 4}\right) .
$$

Beweis. Mit $G_{\lambda}(t):=\int_{1}^{t} F_{\lambda}(y) d y$ gilt

$$
\begin{aligned}
\left|\int_{0}^{a} k_{a}(t) F_{\lambda}(t) d t\right| & =\left|\int_{0}^{a} k_{a}(t) G_{\lambda}^{\prime}(t) d t\right| \\
& =\left|\int_{a-h}^{a} G_{\lambda}(t) k_{a}^{\prime}(t) d t\right| \\
& \leq \int_{a-h}^{a}\left|G_{\lambda}(t)\right|\left|k_{a}^{\prime}(t)\right| d t .
\end{aligned}
$$

Wegen $k_{a}^{\prime}(t) \leq 0$ ist

$$
\int_{a-h}^{a}\left|k_{a}^{\prime}(t)\right| d t=k_{a}\left(a-h_{1}\right)-k_{a}(a)=1
$$


und es gilt also

$$
\left|h_{k_{a}}(\lambda)\right| \leq\left|G_{\lambda}(a)\right| .
$$

Zusammen mit Lemma A.2.2 folgt die Behauptung.

Die folgende Abschätzung benutzt die speziellen Eigenschaften des Kerns $k_{a}$ und die Legendre-Differentialgleichung, um Abschätzungen für $h_{k_{a}}$ zu bekommen, die über das Spektralmaß integrierbar sind. Huber beweist in [15] eine solche Abschätzung für eine Familie von Funktionen (in der unsere Funktion $k_{a}$ enthalten ist) unter Angabe von Konstanten. Seine Funktionenfamilie ist besonders dazu geeignet, diese Konstanten klein zu halten. Für ein Ergebnis -wie das aus Theorem B- ist unsere feste Wahl von $k_{a}$ völlig ausreichend.

Lemma 3.2.4. Für $\lambda>1$ gilt

$$
\left|h_{k_{a}}(\lambda)\right|=O\left(a^{3 / 2} h^{-1} \lambda^{-5 / 4}\right) .
$$

Beweis. Für $\lambda>1$ ist $(\tanh \pi \sqrt{\lambda-1 / 4})^{-1}$ beschränkt. Nach Lemma A.2.3 gibt es also eine Konstante $C_{1}>0$, sodaß gilt

$$
\left|F_{\lambda}(x)\right| \leq C_{1} \lambda^{-1 / 4}\left(x^{2}-1\right)^{-1 / 4}+C_{1} \lambda^{-1 / 2} x^{1 / 2}\left(x^{2}-1\right)^{-1 / 2} .
$$

Die Legendre Differentialgleichung (A.0.1) läßt sich auch in der Form

$$
\lambda F_{\lambda}(x)=-\frac{d}{d x}\left(\left(x^{2}-1\right) F_{\lambda}^{\prime}(x)\right)
$$

schreiben. Durch zweimalige partielle Integration und unter Benutzung von (3.2.1) folgt

$$
\begin{aligned}
\lambda \int_{1}^{a} k_{a}(x) F_{\lambda}(x) d x & =-\int_{1}^{a} k_{a}(x) \frac{d}{d x}\left(\left(x^{2}-1\right) F_{\lambda}^{\prime}(x)\right) d x \\
& =\int_{a-h}^{a}\left(x^{2}-1\right) k_{a}^{\prime}(x) F_{\lambda}^{\prime}(x) d x \\
& =-\int_{a-h}^{a} F_{\lambda}(x)\left(\left(x^{2}-1\right) k_{a}^{\prime \prime}(x)+2 x k_{a}^{\prime}(x)\right) d x .
\end{aligned}
$$

Zusammen mit (3.2.8) ergibt sich

$$
\left|h_{k_{a}}(\lambda)\right| \leq C_{1} \lambda^{-5 / 4} J(1 / 4)+C_{1} \lambda^{-3 / 2} a^{1 / 2} J(1 / 2)
$$

mit

$$
J(\mu):=\int_{a-h}^{a}\left(x^{2}-1\right)^{-\mu}\left|\left(x^{2}-1\right) k_{a}^{\prime \prime}(x)+2 x k_{a}^{\prime}(x)\right| d x .
$$


Da $k_{a}^{\prime}(x) \leq 0$ und wegen (3.2.1) und (3.2.2) folgt für $\mu \in\{1 / 4,1 / 2\}$,

$$
\begin{aligned}
J(\mu) & \leq \int_{a-h}^{a}\left(x^{2}-1\right)^{-\mu}\left(\left(x^{2}-1\right)\left|k_{a}^{\prime \prime}(x)\right|-2 x k_{a}^{\prime}(x)\right) d x \\
& =\int_{a-h}^{a}\left(x^{2}-1\right)^{1-\mu}\left|k_{a}^{\prime \prime}(x)\right| d x-\frac{1}{1-\mu} \int_{a-h}^{a} k_{a}^{\prime}(x) \frac{d}{d x}\left(x^{2}-1\right)^{1-\mu} d x \\
& \leq 3 \int_{a-h}^{a}\left(x^{2}-1\right)^{1-\mu}\left|k_{a}^{\prime \prime}(x)\right| d x \\
& \leq 3\left(a^{2}-1\right)^{1-\mu} h^{-1} \int_{a-h}^{a}\left|k_{a}^{\prime \prime}(x)\right| d x \\
& \leq 9 a^{2-2 \mu} h^{-1} .
\end{aligned}
$$

Setzt man dieses Ergebnis in (3.2.9) ein, ergibt sich

$$
\left|h_{k_{a}}(\lambda)\right| \leq 9 C_{1} \lambda^{-5 / 4} a^{3 / 2} h^{-1}+9 C_{1} \lambda^{-3 / 2} a^{3 / 2} h^{-1}
$$

und damit die Behauptung.

Um die asymptotische Entwicklung der Gitterpunktzählfunktion zu erhalten, entwickeln wir $K_{a}^{b}(z, w)$ und $K_{a}^{\sharp}(z, w)$ in Eigenfunktionen des Laplaceoperators. Der Fehlerterm in der asymptotischen Entwicklung der Gitterpunktzählfunktion wird durch den Anteil in der Entwicklung in Eigenfunktionen durch Eigenwerte größer gleich 1/4 bestimmt. Wir definieren

$$
R(a, h):=\int_{1 / 4}^{\infty}|e(\lambda, z) \overline{e(\lambda, w)}|\left|h_{k_{a}}(\lambda)\right| d \mu(\lambda)
$$

und zeigen:

Lemma 3.2.5. Es gibt eine Konstante $X_{0}>0$ und eine Funktion $R(a, h, s)$, sodaß für alle $s>X_{0}$ und für alle $a, h>1, h<a$ mit $a=\cosh \rho$ gilt, daß

$$
R(a, h) \leq R(a, h, s)=O\left(a^{3 / 2} s^{-1 / 4} h^{-1}\right)+O\left(a^{1 / 2} s^{1 / 4}\right)+O\left(\rho a^{1 / 2}\right) .
$$

Beweis. Mit $r:=\min \{\operatorname{inj}(z), \operatorname{inj}(w)\}, X_{0}:=\max \left\{1, \sinh ^{-2}(r / 2)\right\}$ und

$$
p(\lambda, z, w):=e(\lambda, z) \overline{e(\lambda, w)}
$$

definieren wir

$$
\begin{aligned}
& R_{1}:=\int_{1 / 4}^{X_{0}}|p(\lambda, z, w)|\left|h_{k_{a}}(\lambda)\right| d \mu(\lambda), \\
& R_{2}:=\int_{X_{0}}^{s}|p(\lambda, z, w)|\left|h_{k_{a}}(\lambda)\right| d \mu(\lambda), \\
& R_{3}:=\int_{s}^{\infty}|p(\lambda, z, w)|\left|h_{k_{a}}(\lambda)\right| d \mu(\lambda),
\end{aligned}
$$


sodaß also $R(a, h)=R(a, h, s)=R_{1}+R_{2}+R_{3}$ ist. Aus Korollar 3.1.4 und Lemma 3.2.2 folgt mit $a=\cosh \rho$

$$
R_{1} \leq 8 \rho a^{1 / 2} \cdot \frac{3}{4 \pi} X_{0}=O\left(\rho a^{1 / 2}\right) .
$$

Aus Korollar 3.1.4 folgt, daß es eine Konstante $C_{1}>0$ gibt mit

$$
G(x):=\int_{0}^{x}|p(\lambda, z, w)| d \mu(\lambda) \leq C_{1} x \quad \text { für } \quad x \geq X_{0} .
$$

Für eine Funktion $f \in C^{1}[a, b]$ mit $f(\lambda)>0$ und $f^{\prime}(\lambda) \leq 0$ für $\lambda \in[a, b]$ folgt durch partielle Integration:

$$
\begin{aligned}
\int_{a}^{b} f(\lambda)|p(\lambda, z, w)| d \mu(\lambda) & =\int_{a}^{b} f(x) d G(x) \\
& \leq f(b) G(b)+\int_{a}^{b}\left(-f^{\prime}(x)\right) G(x) d x \\
& \leq C_{1} f(a) a+C_{1} \int_{a}^{b} f(x) d x
\end{aligned}
$$

Aus Lemma 3.2.3 folgt, daß es eine Konstante $C_{2}>0$ gilt, sodaß $\left|h_{k_{a}}(\lambda)\right| \leq C_{2} a^{1 / 2} \lambda^{-3 / 4}$ für $\lambda>1$. Mit (3.2.10) und der Wahl $f(\lambda)=C_{2} a^{1 / 2} \lambda^{-3 / 4}$ folgt

$$
\begin{aligned}
R_{2} & \leq C_{2} \int_{X_{0}}^{s} a^{1 / 2} \lambda^{-3 / 4} d \mu(\lambda) \\
& \leq C_{1} C_{2} a^{1 / 2} s^{1 / 4}+C_{1} C_{2} a^{1 / 2}\left[3 x^{1 / 4}\right]_{X_{0}}^{s} \\
& =O\left(a^{1 / 2} s^{1 / 4}\right) .
\end{aligned}
$$

Nach Lemma 3.2.1 ist

$$
\left|h_{k_{a}}(\lambda)\right| \leq C_{3} a^{3 / 2} h^{-1} \lambda^{-5 / 4}
$$

für eine Konstante $C_{3}>0$. Aus 3.2.10 folgt

$$
\begin{aligned}
R_{3} & \leq C_{3} \int_{s}^{\infty} a^{3 / 2} h^{-1} \lambda^{-3 / 4} d \mu(\lambda) \\
& \leq C_{1} C_{3} a^{3 / 2} h^{-1} s^{-1 / 4}+C_{1} C_{3} a^{3 / 2} h^{-1} \lim _{T \rightarrow \infty}\left[-5 x^{-1 / 4}\right]_{s}^{T} \\
& =O\left(a^{3 / 2} h^{-1} s^{-1 / 4}\right) .
\end{aligned}
$$

Damit ist das Lemma bewiesen

Wir können jetzt Theorem B beweisen.

Beweis von Theorem B. Es sei $G$ eine Fuchsche Gruppe mit Konvergenzexponenten $\delta>1 / 2$. Nach Patterson [30] hat der Laplace-Beltrami Operator auf $D / G$ den kleinsten Eigenwert $\lambda_{1}=\delta(1-\delta)$. Die zugehörige normierte Eigenfunktion $\phi_{1}(z)$ läßt sich über 
das Patterson Maß konstruieren, sie ist reell, positiv und liegt in $L^{2}(D / G)$. Lax und Phillips zeigen in [19], daß das Spektrum des Laplace-Beltrami Operators in [0,1/4) aus nur endlich vielen diskreten Eigenwerten besteht. Diese Eigenwerte seien mit $\lambda_{1}, \lambda_{2}, \ldots, \lambda_{n} \in$ $[0,1 / 4)$ und zugehörigen normierten Eigenfunktionen mit $\phi_{1}, \phi_{2}, \ldots, \phi_{n} \in L^{2}(D / G)$ bezeichnet. Die Funktionen $\phi_{2}, \ldots, \phi_{n}$ sind ebenfalls reell, nehmen jedoch stets auch negative Werte an (siehe Abschnitt 3.1.2). Wegen Lemma 3.2.4 folgt aus Theorem 3.1.5, daß die Spektralzerlegungen von $K_{a}^{b}(z, w)$ und $K_{a}^{\sharp}(z, w)$ absolut konvergieren. Mit $p(\lambda, z, w):=e(\lambda, z) \overline{e(\lambda, w)}$ gilt

$$
K_{a}^{b}(z, w)=\int_{0}^{\infty} p(\lambda, z, w) h_{k_{a}}(\lambda) d \mu(\lambda) .
$$

Wir definieren

$$
C_{i}(z, w):=2 \sqrt{\pi} \frac{\Gamma\left(s\left(\lambda_{i}\right)-1 / 2\right)}{\Gamma\left(1+s\left(\lambda_{i}\right)\right)} \phi_{i}(z) \phi_{i}(w) .
$$

Aus Lemma 3.2.1 und $s\left(\lambda_{1}\right)=\delta$ folgt

$$
\begin{aligned}
\int_{0}^{1 / 4} p(\lambda, z, w) h_{k_{a}}(\lambda) d \mu(\lambda) & =\sum_{i=1}^{n} h_{k_{a}}\left(\lambda_{i}\right) \phi_{i}(z) \phi_{i}(w) \\
& =\sum_{i=1}^{n} C_{i}(z, w) a^{s\left(\lambda_{i}\right)}+O\left(h a^{\delta-1}\right)+O\left(a^{1 / 2}\right)
\end{aligned}
$$

und mit Lemma 3.2.5 gilt unter den Voraussetzungen $a>1,0<h<a, s>X_{0}$, daß

$$
\begin{aligned}
K_{a}^{b}(z, w) \geq & \int_{0}^{1 / 4} p(\lambda, z, w) h_{k_{a}}(\lambda) d \mu(\lambda)-R(a, h, s) \\
= & \sum_{i=1}^{n} C_{i}(z, w) a^{s\left(\lambda_{i}\right)}+O\left(h a^{\delta-1}\right)+O\left(a^{1 / 2}\right)-R(a, h, s) \\
= & \sum_{i=1}^{n} C_{i}(z, w) a^{s\left(\lambda_{i}\right)}+O\left(h a^{\delta-1}\right)+O\left(a^{3 / 2} s^{-1 / 4} h^{-1}\right) \\
& +O\left(a^{1 / 2} s^{1 / 4}\right)+O\left(\rho a^{1 / 2}\right)
\end{aligned}
$$

Wir wählen nun

$$
h=a^{2 / 3(2-\delta)}, \quad s=a^{2 / 3(2 \delta-1)} .
$$

Da $\delta>1 / 2$ vorausgesetzt ist, gilt für $a>1$ sicherlich $h<a$. Für große $a$ ist ebenfalls $s>X_{0}$ erfüllt, und wir erhalten

$$
K_{a}^{b}(z, w) \geq \sum_{i=1}^{n} 2 \sqrt{\pi} \frac{\Gamma\left(s\left(\lambda_{i}\right)-1 / 2\right)}{\Gamma\left(1+s\left(\lambda_{i}\right)\right)} \phi_{i}(z) \phi_{i}(w) a^{s\left(\lambda_{i}\right)}+O\left(a^{1 / 3(\delta+1)}\right) .
$$

Die umgekehrte Richtung für $K_{a}^{\sharp}(z, w)$ zeigt man ganz analog. Es gilt

$$
K_{a}^{\sharp}(z, w)=\int_{0}^{\infty} p(\lambda, z, w) h_{k_{a+h}}(\lambda) d \mu(\lambda) .
$$


Mit Lemma 3.2.1 und $s\left(\lambda_{1}\right)=\delta$ folgt

$$
\begin{aligned}
\int_{0}^{1 / 4} p(\lambda, z, w) h_{k_{a+h}}(\lambda) d \mu(\lambda) & =\sum_{i=1}^{n} h_{k_{a+h}}\left(\lambda_{i}\right) \phi_{i}(z) \phi_{i}(w) \\
& =\sum_{i=1}^{n} C_{i}(z, w) a^{s\left(\lambda_{i}\right)}+O\left(h a^{\delta-1}\right)+O\left(a^{1 / 2}\right) .
\end{aligned}
$$

Für $0<h<a$ gilt $R(a+h, h, s)=O(R(a, h, s))$ und mit Lemma 3.2.5 gilt unter den Voraussetzungen $a>1,0<h<a, s>X_{0}$, daß

$$
\begin{aligned}
K_{a}^{\sharp}(z, w) \leq & \int_{0}^{1 / 4} p(\lambda, z, w) h_{k_{a+h}}(\lambda) d \mu(\lambda)+R(a+h, h, s) \\
= & \sum_{i=1}^{n} C_{i}(z, w) a^{s\left(\lambda_{i}\right)}+O\left(h a^{\delta-1}\right)+O\left(a^{1 / 2}\right)+R(a+h, h, s) \\
= & \sum_{i=1}^{n} C_{i}(z, w) a^{s\left(\lambda_{i}\right)}+O\left(h a^{\delta-1}\right)+O\left(a^{3 / 2} s^{-1 / 4} h^{-1}\right) \\
& +O\left(a^{1 / 2} s^{1 / 4}\right)+O\left(\rho a^{1 / 2}\right) .
\end{aligned}
$$

Wir wählen genau wie oben

$$
h=a^{2 / 3(2-\delta)}, \quad s=a^{2 / 3(2 \delta-1)} .
$$

Die Voraussetzungen an $h$ und $s$ sind für große $a$ wieder erfüllt, und es folgt

$$
K_{a}^{\sharp}(z, w) \leq \sum_{i=1}^{n} 2 \sqrt{\pi} \frac{\Gamma\left(s\left(\lambda_{i}\right)-1 / 2\right)}{\Gamma\left(1+s\left(\lambda_{i}\right)\right)} \phi_{i}(z) \phi_{i}(w) a^{s\left(\lambda_{i}\right)}+O\left(a^{1 / 3(\delta+1)}\right) .
$$

Aus 3.2.3 folgt damit die Behauptung von Theorem B.

\subsection{Numerische Abschätzungen der Zählfunktion}

In diesem Kapitel sollen nun numerische Abschätzungen der Gitterpunktzählfunktion gegeben werden.

Theorem C. Es sei G eine Fuchssche Gruppe mit genau einem Eigenwert $\lambda_{1}=\delta(1-\delta)$ im Intervall $[0,1 / 4)$. Die zugehörige normierte Eigenfunktion sei mit $\phi$ bezeichnet. Es sei $z \in D$ und

$$
\begin{gathered}
r:=\operatorname{inj}(z)>0 \\
\nu(r):= \begin{cases}\frac{5}{4} & \text { falls } \sinh ^{-2} \frac{r}{2}<\frac{5}{4} \\
\sinh ^{-2} \frac{r}{2} & \text { falls } \sinh ^{-2} \frac{r}{2} \geq \frac{5}{4}\end{cases} \\
X_{0}:=\sqrt{\nu(r)-1 / 4},
\end{gathered}
$$




$$
C:=2 \sqrt{\pi} \frac{\Gamma(\delta-1 / 2)}{\Gamma(1+\delta)} \phi^{2}(z), \quad C^{\prime}:=\phi^{2}(z) .
$$

Dann gilt für alle $C_{0}>0$ und mit $\rho>0$ definiert durch $a=\cosh \rho$ und für alle $a \in \mathbb{R}$ mit

$$
\begin{gathered}
a>\max \left\{6,0,6 \cdot C_{0}^{2 / 3}\right\}, \\
a>\left(C_{0}^{-2 / 3} \cdot \max \left\{7,8,1,6 \cdot X_{0}\right\}\right)^{\frac{2}{2 \delta-1}},
\end{gathered}
$$

$d a \beta$

$$
\begin{aligned}
N(a ; z, w) \leq & C a^{\delta}+\left(3,9 \cdot C C_{0}^{-2 / 3}+11,1 \cdot C_{0}^{1 / 3}\right) \cdot a^{1 / 3(1+\delta)} \\
& +18 \cdot C_{0}^{-1 / 3} a^{1 / 3(2-\delta)}+19 \cdot C_{0}^{-2 / 3} a^{1 / 6(5-4 \delta)} \\
& +\left(1.5 \cdot \log C_{0}+0,8 \cdot \log a+3 \cdot \nu(r) \rho+27\right) \cdot a^{1 / 2}+3 C^{\prime} .
\end{aligned}
$$

In Theorem D wird die Zählfunktion nach unten abgeschätzt.

Theorem D. Es sei G eine geometrisch endliche Fuchssche Gruppe mit Konvergenzexponenten $\delta>1 / 2$. Die normierte Eigenfunktion zum kleinsten Eigenwert $\lambda_{1}=\delta(1-\delta)$ sei $\phi \in L^{2}(D / G)$. Weiter sei $z \in D, r:=\operatorname{inj}(z)>0$ und $\nu, X_{0}$ wie in Theorem $C$,

$$
C:=2 \sqrt{\pi} \frac{\Gamma(\delta-1 / 2)}{\Gamma(1+\delta)} \phi^{2}(z), \quad C^{\prime}:=\phi^{2}(z) .
$$

Dann gilt für alle $C_{0}>0$ und mit $\rho>0$ definiert durch $a=\cosh \rho$ und für alle $a \in \mathbb{R}$ mit

$$
\begin{gathered}
a>\max \left\{6,0,6 \cdot C_{0}^{2 / 3}\right\}, \\
a>\left(C_{0}^{-2 / 3} \cdot \max \left\{7,8,1,6 \cdot X_{0}\right\}\right)^{\frac{2}{2 \delta-1}},
\end{gathered}
$$

$d a \beta$

$$
\begin{aligned}
N(a ; z, z) \geq & C a^{\delta}-\left(3,9 \cdot C C_{0}^{-2 / 3}+7,8 \cdot C_{0}^{1 / 3}\right) \cdot a^{1 / 3(1+\delta)} \\
& -3,2 \cdot C_{0}^{-1 / 3} a^{1 / 3(2-\delta)}-6,7 \cdot C_{0}^{-2 / 3} a^{1 / 6(5-4 \delta)} \\
& -\left(\log C_{0}+\frac{1}{2} \log a+2 \cdot \nu(r) \rho+19\right) \cdot a^{1 / 2} \\
& -4 C^{\prime}\left(2+\left(\delta-\frac{1}{2}\right)^{-1}\right) a^{(1-\delta)}-1,5 C-6 C^{\prime} .
\end{aligned}
$$

Um die in den Abschätzungen auftretenden Konstanten möglichst klein zu halten, definieren wir den Kern $k_{a}$ aus dem vorherigen Kapitel etwas anders. Wie Huber in [15] erklären wir eine Funktion $b$ durch

$$
b(x):=\int_{x}^{1} y^{\epsilon}(1-y)^{\epsilon} d y / I(\epsilon), \quad 0 \leq x \leq 1,
$$




$$
I(\epsilon):=\int_{0}^{1} y^{\epsilon}(1-y)^{\epsilon} d y, \quad \epsilon>0 .
$$

Diese Funktion hat die folgenden Eigenschaften:

$$
\begin{aligned}
& 1=b(0) \geq b(x) \geq b(1)=0 \quad \text { für } \quad 0 \leq x \leq 1, \\
& b^{\prime}(0)=b^{\prime}(1)=0, \\
& b^{\prime}\left(\frac{1}{2}\right)=-1 /\left(4^{\epsilon} I(\epsilon)\right), \\
& b^{\prime \prime}(x)<0 \quad \text { für } \quad x \in\left(0, \frac{1}{2}\right), \quad b^{\prime \prime}(x)>0 \quad \text { für } \quad x \in\left(\frac{1}{2}, 1\right) \text {, } \\
& \int_{0}^{1} b(x) d x=\frac{1}{2} .
\end{aligned}
$$

Nun definieren wir für $0<h<a$ die Funktion $k_{a} \in C_{0}^{1}[0, \infty)$ durch

$$
k_{a}(r)= \begin{cases}1 & \text { für } 0 \leq r \leq a-h, \\ b\left(\frac{r-a+h}{h}\right) & \text { für } a-h<r \leq a, \\ 0 & \text { für } r>a .\end{cases}
$$

Mit

$$
\begin{aligned}
K_{a}^{b}(z, w) & :=\sum_{g \in G} k_{a}(\cosh d(g(z), w)), \\
K_{a}^{\sharp}(z, w) & :=\sum_{g \in G} k_{a+h}(\cosh d(g(z), w))
\end{aligned}
$$

gilt wieder

$$
K_{a}^{b}(z, w) \leq N(a, z, w) \leq K_{a}^{\sharp}(z, w) .
$$

Um numerische Abschätzungen für $K^{b}$ und $K^{\sharp}$ zu bekommen, gehen wir wie in Kapitel 3.2 vor, wobei wir allerdings alle Konstanten, die in den Ordnungsabschätzungen verschwinden, explizit berechnen und angeben.

Die folgenden beiden Lemmata geben Abschätzungen für die Spektralfunktion $h_{k_{a}}$ für $\lambda>1 / 4$ wie Huber sie in [15] beweist.

Lemma 3.3.1. Für $\lambda>1 / 4$ gilt

$$
\begin{gathered}
\left|h_{k_{a}}(\lambda)\right| \leq \frac{4 \sqrt{2} a^{1 / 2}}{\kappa(\lambda)}, \quad \text { für } \quad a>1, \\
\left|h_{k_{a}}(\lambda)\right| \leq\left(\frac{2 \pi \alpha}{\kappa^{3 / 2}(\lambda)}+\frac{2 \pi \gamma}{\kappa^{2}(\lambda)}\right) a^{1 / 2}, \quad \text { für } \quad a>3, \\
\kappa(\lambda):=\left(\lambda-\frac{1}{4}\right)^{1 / 2}, \quad \alpha:=\sqrt{\frac{2}{\pi}}, \quad \gamma:=\frac{289}{144} \frac{\sqrt{2}}{\pi} .
\end{gathered}
$$


Beweis. Mit $G_{\lambda}(t):=\int_{1}^{t} F_{\lambda}(y) d y$ gilt

$$
\begin{aligned}
\left|\int_{0}^{a} k_{a}(t) F_{\lambda}(t) d t\right| & =\left|\int_{0}^{a} k_{a}(t) G_{\lambda}^{\prime}(t) d t\right| \\
& =\left|\int_{a-h}^{a} G_{\lambda}(t) k_{a}^{\prime}(t) d t\right| \\
& \leq \int_{a-h}^{a}\left|G_{\lambda}(t)\right|\left|k_{a}^{\prime}(t)\right| d t .
\end{aligned}
$$

Wegen $k_{a}^{\prime}(t) \leq 0$ ist

$$
\int_{a-h}^{a}\left|k_{a}^{\prime}(t)\right| d t=k_{a}(a-h)-k_{a}(a)=1
$$

und es gilt also

$$
\left|h_{k_{a}}(\lambda)\right| \leq\left|G_{\lambda}(a)\right| .
$$

Zusammen mit Lemma A.2.2 folgt die Behauptung.

Die folgende Abschätzung benutzt die speziellen Eigenschaften des Kerns $k_{a}$ und die Legendre-Differentialgleichung, um eine Abschätzung für $h_{k_{a}}$ zu gewinnen, die über das Spektralmaß integrierbar ist.

Lemma 3.3.2. Für $\lambda>1 / 4$ und mit $a>5,2 \leq h<a-3$ gilt

$$
\begin{aligned}
& \tanh \pi \kappa\left|h_{k_{a}}(\lambda)\right| \leq \frac{4 \pi \alpha}{4^{\epsilon} I(\epsilon)}\left(\frac{a^{3 / 2}}{h}+\frac{1}{3} a^{1 / 2}\right) \frac{1}{\kappa^{1 / 2}\left(\kappa^{2}+1 / 4\right)} \\
&+\frac{4 \pi \beta}{4^{\epsilon} I(\epsilon)}\left(\frac{a^{3 / 2}}{h}+a^{1 / 2}\right) \frac{1}{\kappa\left(\kappa^{2}+1 / 4\right)} \\
& \kappa=\kappa(\lambda):=\left(\lambda-\frac{1}{4}\right)^{1 / 2}, \quad \alpha:=\sqrt{\frac{2}{\pi}}, \quad \beta:=\frac{19}{18} \frac{\sqrt{2}}{\pi} .
\end{aligned}
$$

Beweis. Wir folgen Huber [15] S. 137-138. Die Legendre Differentialgleichung A.0.1 läßt sich auch in der Form

$$
\lambda F_{\lambda}(x)=-\frac{d}{d x}\left(\left(x^{2}-1\right) F_{\lambda}^{\prime}(x)\right)
$$

schreiben. Durch zweimalige partielle Integration und unter Benutzung von (3.3.1) und (3.3.2) folgt

$$
\begin{aligned}
\lambda \int_{1}^{a} k_{a}(x) F_{\lambda}(x) d x & =-\int_{1}^{a} k_{a}(x) \frac{d}{d x}\left(\left(x^{2}-1\right) F_{\lambda}^{\prime}(x)\right) d x \\
& =\int_{a-h}^{a}\left(x^{2}-1\right) k_{a}^{\prime}(x) F_{\lambda}^{\prime}(x) d x \\
& =-\int_{a-h}^{a} F_{\lambda}(x)\left(\left(x^{2}-1\right) k_{a}^{\prime \prime}(x)+2 x k_{a}^{\prime}(x)\right) d x
\end{aligned}
$$


Zusammen mit A.2.3 ergibt sich

$$
\left|h_{k_{a}}(\lambda)\right| \leq \frac{\alpha}{\kappa^{1 / 2}\left(\kappa^{2}+1 / 4\right)} J(1 / 4)+\frac{\beta a^{1 / 2}}{\kappa\left(\kappa^{2}+1 / 4\right)} J(1 / 2)
$$

mit

$$
J(\mu):=\int_{a-h}^{a}\left(x^{2}-1\right)^{-\mu}\left|\left(x^{2}-1\right) k_{a}^{\prime \prime}(x)+2 x k_{a}^{\prime}(x)\right| d x .
$$

Da $k_{a}^{\prime}(x) \leq 0$ und mit Eigenschaft 3.3.2 folgt

$$
\begin{aligned}
J(\mu) & \leq \int_{a-h}^{a}\left(x^{2}-1\right)^{-\mu}\left(\left(x^{2}-1\right)\left|k_{a}^{\prime \prime}(x)\right|-2 x k_{a}^{\prime}(x)\right) d x \\
& =\int_{a-h}^{a}\left(x^{2}-1\right)^{1-\mu}\left|k_{a}^{\prime \prime}(x)\right| d x-\frac{1}{1-\mu} \int_{a-h}^{a} k_{a}^{\prime}(x) \frac{d}{d x}\left(x^{2}-1\right)^{1-\mu} d x \\
& =\int_{a-h}^{a}\left(x^{2}-1\right)^{1-\mu}\left(\left|k_{a}^{\prime \prime}(x)\right|-\frac{1}{1-\mu} k_{a}^{\prime \prime}(x)\right) d x .
\end{aligned}
$$

Mit (3.3.4) ergibt sich

$$
\begin{aligned}
J(\mu) \leq & \frac{\mu}{1-\mu} \int_{a-h}^{a-h / 2}\left(x^{2}-1\right)^{1-\mu} k_{a}^{\prime \prime}(x) d x+\frac{2-\mu}{1-\mu} \int_{a-h / 2}^{a}\left(x^{2}-1\right)^{1-\mu} k_{a}^{\prime \prime}(x) d x \\
\leq & \frac{\mu}{1-\mu}\left((a-h)^{2}-1\right)^{1-\mu} \int_{a-h}^{a-h / 2} k_{a}^{\prime \prime}(x) d x \\
& +\frac{2-\mu}{1-\mu}\left(a^{2}-1\right)^{1-\mu} \int_{a-h / 2}^{a} k_{a}^{\prime \prime}(x) d x,
\end{aligned}
$$

und mit (3.3.3) folgt

$$
J(\mu) \leq \frac{\left(a^{2}-1\right)^{1-\mu}}{4^{\epsilon} I(\epsilon) h}\left(\frac{2-\mu}{1-\mu}-\frac{\mu}{1-\mu}\left(\frac{(a-h)^{2}-1}{a^{2}-1}\right)^{1-\mu}\right) .
$$

Wegen $h<a-3$ gilt

$$
0<\frac{(a-h)^{2}-1}{a^{2}-1}<1,
$$

und da $0<1-\mu<1$ ergibt sich

$$
\left(\frac{(a-h)^{2}-1}{a^{2}-1}\right)^{1-\mu}>\frac{(a-h)^{2}-1}{a^{2}-1}=1-\frac{h(2 a-h)}{a^{2}-1} .
$$

Schließlich folgt mit $h \geq 2$

$$
\begin{aligned}
J(\mu) & \leq \frac{\left(a^{2}-1\right)^{1-\mu}}{4^{\epsilon} I(\epsilon)}\left(\frac{2}{h}+\frac{2 \mu}{1-\mu} \frac{1}{a+1}\right) \\
& \leq \frac{2}{4^{\epsilon} I(\epsilon)}\left(\frac{a^{2(1-\mu)}}{h}+\frac{\mu}{1-\mu} a^{1-2 \mu}\right) .
\end{aligned}
$$

Zusammen mit 3.3.7 ergibt sich die Behauptung. 
Der Eigenwert $\lambda=1 / 4$ bedarf einer besonderen Betrachtung.

Lemma 3.3.3. Mit $a=\cosh \rho, a>1, \lambda \geq 1 / 4$ gilt

$$
\left|h_{k_{a}}(\lambda)\right| \leq 8 \rho a^{1 / 2} .
$$

Beweis. Aus den Integraldarstellungen A.0.2 und A.0.3 für $F_{\lambda}$ folgt $\left|F_{\lambda}(t)\right| \leq F_{1 / 4}(t)$ für $t>1, \lambda \geq 1 / 4$. Damit folgt

$$
\begin{aligned}
\left|h_{k_{a}}(\lambda)\right| & =2 \pi\left|\int_{1}^{a} F_{\lambda}(t) k_{a}(t) d t\right| \\
& \leq 2 \pi \int_{1}^{a}\left|F_{\lambda}(t)\right| k_{a}(t) d t \\
& \leq 2 \pi \int_{1}^{a} F_{1 / 4}(t) k_{a}(t) d t=h_{k_{a}}(\lambda) \\
& \leq 2 \pi \int_{1}^{a} F_{1 / 4}(t) d t .
\end{aligned}
$$

Die Behauptung folgt nun aus Lemma A.2.1.

Die Eigenwerte kleiner als 1/4 geben uns später den führenden Term in der Asymptotik der Gitterpunktzählfunktion.

Lemma 3.3.4. Für $0<\lambda<1 / 4, a>1$ und mit $s(\lambda):=\frac{1}{2}+\sqrt{\frac{1}{4}-\lambda}$ gilt

$$
\begin{gathered}
h_{k_{a}}(\lambda) \leq C a^{s(\lambda)}+3 a^{-\frac{1}{2}} \\
h_{k_{a}}(\lambda) \geq C(a-h)^{s(\lambda)}-4\left(2+\kappa^{-1}\right)(a-h)^{(1-s(\lambda))} \\
-C(a-h)^{(s(\lambda)-1)}-6(a-h)^{-\frac{3}{2}}-\frac{1}{2} C \\
C=2 \sqrt{\pi} \frac{\Gamma\left(s(\lambda)-\frac{1}{2}\right)}{\Gamma(1+s(\lambda))} .
\end{gathered}
$$

Um die Rechnungen zu vereinfachen, machen wir die Variablentransformation

$$
\kappa(\lambda)=\sqrt{\lambda-1 / 4}, \quad \text { für } \quad \lambda>1 / 4
$$

und definieren

$$
\widetilde{h}_{k_{a}}(\kappa):=h_{k_{a}}\left(1 / 4+\kappa^{2}\right) \quad \text { für } \quad \kappa \geq 0 .
$$

Wie in Kapitel 3.2 beginnen wir mit der Abschätzung des Anteils in der Entwicklung in Eigenfunktionen, welcher durch Eigenwerte größer gleich 1/4 zustande kommt.

$$
p(\lambda, z, w):=e(\lambda, z) \overline{e(\lambda, w)}
$$




$$
\begin{aligned}
R(a, h) & :=\int_{1 / 4}^{\infty}|p(\lambda, z, w)|\left|h_{k_{a}}(\lambda)\right| d \mu(\lambda) \\
& =\int_{0}^{\infty}\left|p\left(1 / 4+\kappa^{2}, z, w\right)\right|\left|\widetilde{h}_{k_{a}}(\kappa)\right| 2 \kappa d \mu(\kappa) .
\end{aligned}
$$

Aus Korollar 3.1.4 folgt mit

$$
G(x):=\int_{0}^{x}\left|p\left(1 / 4+\kappa^{2}, z, w\right)\right| 2 \kappa d \mu(\kappa) .
$$

Korollar 3.3.5. Für $x \in \mathbb{R}^{+}$mit $x^{2} \geq \sinh ^{-2} \frac{r}{2}-1 / 4, r:=\min \{\operatorname{inj}(z), \operatorname{inj}(w)\}$ gilt

$$
G(x) \leq \frac{3}{4 \pi}\left(1 / 4+x^{2}\right) .
$$

Beweis. Wegen Korollar 3.1.4 gilt mit $x$, wie oben gefordert, daß

$$
\begin{aligned}
\int_{1 / 4}^{1 / 4+x^{2}}|p(\lambda, z, w)| d \mu(\lambda) & =\int_{0}^{x}\left|p\left(1 / 4+\kappa^{2}, z, w\right)\right| 2 \kappa d \mu(\kappa) \\
& \leq \frac{3}{4 \pi}\left(1 / 4+x^{2}\right) .
\end{aligned}
$$

Lemma 3.3.6. Es seinen $z, w \in D$ und

$$
\begin{gathered}
r:=\min \{\operatorname{inj}(z), \operatorname{inj}(w)\} \\
\nu(r):= \begin{cases}\frac{5}{4} & \text { falls } \sinh ^{-2} \frac{r}{2}<\frac{5}{4} \\
\sinh ^{-2} \frac{r}{2} & \text { falls } \sinh ^{-2} \frac{r}{2} \geq \frac{5}{4}\end{cases} \\
X_{0}:=\sqrt{\nu(r)-1 / 4 .}
\end{gathered}
$$

Für $s>X_{0}, a>3$ und mit $\rho>0$ definiert durch $a=\cosh \rho$ gilt

$$
\begin{gathered}
R(a, h) \leq R(a, h, s) \text { mit } \\
R(a, h, s):=\frac{1}{4^{\epsilon} I(\epsilon)}\left(\frac{15 \alpha a^{3 / 2}}{h s^{1 / 2}}+\frac{9 \beta a^{3 / 2}}{h s}+\frac{5 \alpha a^{1 / 2}}{s^{1 / 2}}+\frac{9 \beta a^{1 / 2}}{s}\right) \\
+a^{1 / 2}\left(6 \alpha s^{1 / 2}+3 \gamma \log s+10^{-2} s^{-1}+\frac{6}{\pi} \nu(r) \rho+\frac{15 \gamma}{8}-\frac{33 \alpha}{8}\right), \\
\alpha:=\sqrt{\frac{2}{\pi}}, \quad \beta:=\frac{19}{18} \frac{\sqrt{2}}{\pi}, \quad \gamma:=\frac{289}{144} \frac{\sqrt{2}}{\pi} .
\end{gathered}
$$


Beweis. Wir definieren

$$
\begin{aligned}
& R_{1}:=\int_{0}^{X_{0}}\left|p\left(1 / 4+\kappa^{2}, z, w\right)\right|\left|\widetilde{h}_{k_{a}}(\kappa)\right| 2 \kappa d \mu(\kappa), \\
& R_{2}:=\int_{X_{0}}^{s}\left|p\left(1 / 4+\kappa^{2}, z, w\right)\right|\left|\widetilde{h}_{k_{a}}(\kappa)\right| 2 \kappa d \mu(\kappa), \\
& R_{3}:=\int_{s}^{\infty}\left|p\left(1 / 4+\kappa^{2}, z, w\right)\right|(1-\tanh \pi \kappa)\left|\widetilde{h}_{k_{a}}(\kappa)\right| 2 \kappa d \mu(\kappa), \\
& R_{4}:=\int_{s}^{\infty}\left|p\left(1 / 4+\kappa^{2}, z, w\right)\right| \tanh \pi \kappa\left|\widetilde{h}_{k_{a}}(\kappa)\right| 2 \kappa d \mu(\kappa) .
\end{aligned}
$$

Es gilt also $R(a, h)=R_{1}+R_{2}+R_{3}+R_{4}$. Für eine Funktion $f \in C^{1}[a, b]$ mit $f(\lambda)>0$ und $f^{\prime}(\lambda) \leq 0$ für $\lambda \in[a, b]$ folgt mit $\lambda=1 / 4+\kappa$ durch partielle Integration.

$$
\begin{aligned}
\int_{\kappa_{1}}^{\kappa_{2}} f(\lambda)|p(\lambda, z, w)| 2 \kappa d \mu(\kappa) & =\int_{\kappa_{1}}^{\kappa_{2}} f(x) d G(x) \\
& \leq f\left(\kappa_{2}\right) G\left(\kappa_{2}\right)+\int_{\kappa_{1}}^{\kappa_{2}}\left(-f^{\prime}(x)\right) G(x) d x \\
& \leq f\left(\kappa_{1}\right) G\left(\kappa_{1}\right)+\frac{3}{4 \pi} \int_{\kappa_{1}}^{\kappa_{2}} f(x) \frac{d}{d x}\left(\frac{1}{4}+x^{2}\right) d x \\
& \leq \frac{3}{4 \pi}\left(\left(\kappa_{1}^{2}+\frac{1}{4}\right) f\left(\kappa_{1}\right)+2 \int_{\kappa_{1}}^{\kappa_{2}} f(x) x d x\right) .(3.3 .8)
\end{aligned}
$$

Wir schätzen nun $R_{1}, \ldots, R_{4}$ nach oben ab. Für $R_{1}$ ziehen wir Lemma 3.3.3 und Korollar 3.3.5 heran und erhalten

$$
R_{1}(a, h) \leq \frac{6}{\pi} \nu(r) \rho a^{1 / 2}
$$

mit $a=\cosh \rho$. Um $R_{2}$ abzuschätzen, benutzen wir die zweite Abschätzung aus Lemma 3.3.1 und die Ungleichung 3.3.8. Wir halten uns dabei nahe an Huber [15].

$$
\begin{aligned}
R_{2}(a, h, s) \leq & \frac{3}{2}\left(\frac{\alpha\left(X_{0}^{2}+\frac{1}{4}\right)}{X_{0}^{3 / 2}}+\frac{\gamma\left(X_{0}^{2}+\frac{1}{4}\right)}{X_{0}^{2}}\right) a^{1 / 2} \\
& +3\left(\int_{X_{0}}^{s}\left(\alpha \kappa^{-1 / 2}+\gamma \kappa^{-1}\right) d \kappa\right) a^{1 / 2} \\
= & \left(\frac{3}{2} \alpha X_{0}^{1 / 2}+\frac{3}{8} \alpha X_{0}^{-3 / 2}+\frac{3}{2} \gamma+\frac{3}{8} \gamma X_{0}^{-2}\right) a^{1 / 2} \\
& +\left(6 \alpha s^{1 / 2}+3 \gamma \log s-6 \alpha X_{0}^{1 / 2}-3 \log X_{0}\right) a^{1 / 2} \\
= & a^{1 / 2}\left(6 \alpha s^{1 / 2}+3 \gamma \log s+v\left(X_{0}\right)\right) .
\end{aligned}
$$

mit

$$
v(x)=-\frac{9 \alpha}{2} x^{1 / 2}+\frac{3 \alpha}{8} x^{-3 / 2}-3 \gamma \log x+\frac{3 \gamma}{8} x^{-2}+\frac{3}{2} \gamma .
$$


Da $v$ monoton fallend ist für $x>0$ und $X_{0} \geq 1$ gilt

$$
v\left(X_{0}\right) \leq v(1)=\frac{15 \gamma}{8}-\frac{33 \alpha}{8}
$$

Es folgt

$$
R_{2}(a, h, s) \leq a^{1 / 2}\left(6 \alpha s^{1 / 2}+3 \gamma \log s+\frac{15 \gamma}{8}-\frac{33 \alpha}{8}\right)
$$

Der Rest $R_{3}$ hat nur einen sehr kleinen Anteil an $R$. Es gilt

$$
1-\tanh \pi \kappa=\frac{2}{e^{2 \pi \kappa}+1}<2 e^{-2 \pi \kappa} .
$$

Mit der ersten Abschätzung in 3.3.1 und Korollar 3.3.5 folgt

$$
\begin{aligned}
R_{3}(a, h, s) & \leq 8 \sqrt{2} a^{1 / 2} \int_{s}^{\infty} x^{-1} e^{-2 \pi x} d G(x) \\
& \leq 8 \sqrt{2} a^{1 / 2} s^{-1} \int_{s}^{\infty} e^{-2 \pi x} d G(x) \\
& \leq 12 \sqrt{2} a^{1 / 2} s^{-1} \int_{1}^{\infty} e^{-2 \pi x}\left(x^{2}+\frac{1}{4}\right) d x \\
& \leq \frac{\sqrt{2}}{\pi}\left(\frac{15}{2}+\frac{6}{\pi}+\frac{3}{\pi^{2}}\right) e^{-2 \pi} a^{1 / 2} s^{-1} \\
& \leq 10^{-2} a^{1 / 2} s^{-1} .
\end{aligned}
$$

Für $R_{4}$ brauchen wir eine Abschätzung von $k_{h_{a}}$, die ab einem gewissen Punkt über das gesamte Spektrum integrierbar ist. Aus Lemma 3.3.2 und Ungleichung (3.3.8) folgt für $s>X_{0}>1$

$$
\begin{aligned}
R_{4}(a, h, s) \leq & \frac{3 \alpha}{4^{\epsilon} I(\epsilon)}\left(\frac{a^{3 / 2}}{h}+\frac{1}{3} a^{1 / 2}\right)\left(s^{-\frac{1}{2}}+2 \int_{s}^{\infty} \frac{\kappa^{1 / 2}}{\left(\kappa^{2}+1 / 4\right)} d \kappa\right) \\
& +\frac{3 \beta}{4^{\epsilon} I(\epsilon)}\left(\frac{a^{3 / 2}}{h}+a^{1 / 2}\right)\left(s^{-1}+2 \int_{s}^{\infty} \frac{1}{\left(\kappa^{2}+1 / 4\right)} d \kappa\right) \\
\leq & \frac{15 \alpha}{4^{\epsilon} I(\epsilon)}\left(\frac{a^{3 / 2}}{h}+\frac{1}{3} a^{1 / 2}\right) s^{-1 / 2} \\
& +\frac{9 \beta}{4^{\epsilon} I(\epsilon)}\left(\frac{a^{3 / 2}}{h}+a^{1 / 2}\right) s^{-1} \\
= & \frac{1}{4^{\epsilon} I(\epsilon)}\left(\frac{15 \alpha a^{3 / 2}}{h s^{1 / 2}}+\frac{5 \alpha a^{1 / 2}}{s^{1 / 2}}+\frac{9 \beta a^{3 / 2}}{h s}+\frac{9 \beta a^{1 / 2}}{s}\right) .
\end{aligned}
$$

Wir können jetzt Theorem $\mathrm{C}$ und $\mathrm{D}$ beweisen. 
Beweis von Theorem $C$ und $D$. Es sei $G$ eine Fuchssche Gruppe mit Konvergenzexponenten $\delta>1 / 2$, und es gebe außer dem diskreten Eigenwert $\lambda_{1}=\delta(1-\delta)$ keine weiteren Eigenwerte im Intervall $[0,1 / 4)$. Die zu $\lambda_{1}$ gehörige normierte Eigenfunktion $\phi_{1}(z)$ läßt sich über das Patterson Maß konstruieren und liegt in $L^{2}(D / G)$. Wegen Lemma 3.2.4 folgt aus Theorem 3.1.5, daß die Spektralzerlegung von $K_{a}^{\sharp}(z, w)$ absolut konvergiert. Mit $p(\lambda, z, w):=e(\lambda, z) \overline{e(\lambda, w)}$ gilt

$$
K_{a}^{\sharp}(z, w)=\int_{0}^{\infty} p(\lambda, z, w) h_{k_{a+h}}(\lambda) d \mu(\lambda) .
$$

Wir definieren

$$
C:=2 \sqrt{\pi} \frac{\Gamma(\delta-1 / 2)}{\Gamma(1+\delta)} \phi^{2}(z), \quad C^{\prime}:=\phi^{2}(z)
$$

und

$$
\widetilde{R}(a, h, s):=a^{-1 / 2} R(a, h, s) .
$$

Wegen Lemma 3.3.6 gilt

$$
\begin{aligned}
\widetilde{R}(a+h, h, s) \leq & \frac{1}{4^{\epsilon} I(\epsilon)}\left(\frac{15 \alpha a}{h s^{1 / 2}}+\frac{9 \beta a}{h s}+\frac{20 \alpha}{s^{1 / 2}}+\frac{18 \beta}{s}\right) \\
& +6 \alpha s^{1 / 2}+3 \gamma \log s+10^{-2} s^{-1}+\frac{6}{\pi} \nu(r) \rho+\frac{15 \gamma}{8}-\frac{33 \alpha}{8} .
\end{aligned}
$$

Mit Lemma 3.3.4, $(a+h)^{\delta}=a^{\delta}+\delta \int_{a}^{a+h} x^{\delta-1} d x \leq a^{\delta}+\delta h(a+h)^{\delta-1}$ für $0<\delta<1$ und Lemma 3.2.5 gilt

$$
\begin{aligned}
K_{a}^{\sharp}(z, z) & \leq \int_{0}^{1 / 4} p(\lambda, z, z) h_{k_{a+h}}(\lambda) d \mu(\lambda)+R(a+h, h, s) \\
& \leq C(a+h)^{\delta}+3 C^{\prime}(a+h)^{-1 / 2}+(a+h)^{\frac{1}{2}} \widetilde{R}(a+h, h, s) \\
& \leq C a^{\delta}+C h(a+h)^{\delta-1}+(a+h)^{\frac{1}{2}} \widetilde{R}(a+h, h, s)+3 C^{\prime} \\
& \leq C a^{\delta}+C h a^{\delta-1}+(a+h)^{\frac{1}{2}} \widetilde{R}(a+h, h, s)+3 C^{\prime} .
\end{aligned}
$$

Wir lassen nun $\epsilon$ gegen Null gehen. Es gilt

$$
\frac{1}{4^{\epsilon} I(\epsilon)} \rightarrow 1 \quad \text { für } \quad \epsilon \rightarrow 0 .
$$

Der Restterm, der möglichst klein gehalten werden muß, ist

$$
H(a, h, s):=C h a^{\delta-1}+(a+h)^{\frac{1}{2}} \widetilde{R}(a+h, h, s)+3 C^{\prime} .
$$

Wir müssen $h$ und $s$ in Abhängigkeit von $a$ so wählen, daß $H(a, h, s)$ wenigstens für nicht zu kleine $a$ minimal wird. Für hinreichend große $a$ liegt das Minimum von $H(a, h, s)$ unter der Annahme, daß $h(a)$ wesentlich schwächer als $a$ wächst und $s(a) \rightarrow \infty$ für $a \rightarrow \infty$ hinreichend schnell, nahe bei dem Minimum vom

$$
\widetilde{H}(a, h, s):=C h a^{\delta-1}+A h^{-1} s^{-1 / 2}+B s^{1 / 2},
$$




$$
A:=15 \alpha a^{3 / 2}, \quad B:=6 \alpha a^{1 / 2}
$$

liegt. Man betrachte die Wahl von $h$ und $s$ in Kapitel 3.2. Für festes $a$ hat $\widetilde{H}$ sein Minimum für $(s, h)=\left(s_{0}, h_{0}\right)$ mit

$$
\begin{gathered}
s_{0}:=C_{0}^{2 / 3}\left(\frac{A}{B^{2}}\right)^{2 / 3} a^{2 / 3(\delta-1)}=\frac{1}{2} C_{0}^{2 / 3}\left(\frac{25 \pi}{36}\right)^{1 / 3} a^{1 / 3(2 \delta-1)}, \\
h_{0}:=\frac{A}{B} s_{0}^{-1}=5 C_{0}^{-2 / 3}\left(\frac{36}{25 \pi}\right)^{1 / 3} a^{2 / 3(2-\delta)}
\end{gathered}
$$

mit $C_{0}=C$. Da wir keine Kontrolle über $C$ haben, führen wir die frei wählbare Konstante $C_{0}$ ein. Für diese Wahl von $h$ und $s$ wird im Appendix B der Restterm aus (3.3.10) berechnet. Es folgt

$$
\begin{aligned}
K_{a}^{\sharp}(z, z) \leq & C a^{\delta}+\left(3,9 \cdot C C_{0}^{-2 / 3}+11,1 \cdot C_{0}^{1 / 3}\right) \cdot a^{1 / 3(1+\delta)} \\
& +18 \cdot C_{0}^{-1 / 3} a^{1 / 3(2-\delta)}+19 \cdot C_{0}^{-2 / 3} a^{1 / 6(5-4 \delta)} \\
& +\left(1.5 \cdot \log C_{0}+0,8 \cdot \log a+3 \cdot \nu(r) \rho+27\right) \cdot a^{1 / 2}+3 C^{\prime} .
\end{aligned}
$$

Es müssen die folgenden Bedingungen an $a, h_{0}$ und $s_{0}$ erfüllt sein:

$$
\begin{aligned}
a & >5, \\
h_{0} & \geq 2, \\
h_{0} & <a-3, \\
s_{0} & \geq X_{0} .
\end{aligned}
$$

Diese Bedingungen sind erfüllt wenn

$$
\begin{gathered}
a>\max \left\{6,0,6 \cdot C_{0}^{2 / 3}\right\}, \\
a>\left(C_{0}^{-2 / 3} \cdot \max \left\{7,8,1,6 \cdot X_{0}\right\}\right)^{\frac{2}{2 \delta-1}}
\end{gathered}
$$

(siehe Appendix B). Damit ist Theorem C bewiesen. Für den Beweis von Theorem D gehen wir analog vor. Lax und Philiphs zeigen in [19], daß das Spektrum des LaplaceBeltrami Operators in $[0,1 / 4)$ aus nur endlich vielen diskreten Eigenwerten besteht. Diese Eigenwerte seien mit $\lambda_{1}, \lambda_{2}, \ldots, \lambda_{n} \in[0,1 / 4)$ und zugehörigen normierten Eigenfunktionen mit $\phi_{1}, \phi_{2}, \ldots, \phi_{n} \in L^{2}(D / G)$ bezeichnet. Wir definieren

$$
C_{i}(z):=2 \sqrt{\pi} \frac{\Gamma(s(\lambda)-1 / 2)}{\Gamma(1+s(\lambda))} \phi_{i}^{2}(z) .
$$

Es gilt $C_{i}(z)>0$. Alle kleinen Eigenwerte liefern also ausschließlich positive Beiträge zu der Gitterpunktzählfunktion. Es sei

$$
C:=2 \sqrt{\pi} \frac{\Gamma(s(\lambda)-1 / 2)}{\Gamma(1+s(\lambda))} \phi_{1}^{2}(z), \quad C^{\prime}=\phi_{1}^{2}(z) .
$$


Wir benutzen wieder $(a-h)^{\delta}=a^{\delta}-\delta \int_{a-h}^{a} x^{\delta-1} d x \geq a^{\delta}-\delta h a^{\delta-1}$ für $0 \leq \delta<1$. Mit der gleichen Wahl für $h$ und $s$ und $R(a, h, s)$ wie in Lemma 3.3.6 gilt dann

$$
\begin{aligned}
K_{a}^{b}(z, z) \geq & \int_{0}^{1 / 4} p(\lambda, z, z) h_{k_{a}}(\lambda) d \mu(\lambda)-R(a, h, s) \\
\geq & C(a-h)^{\delta}-4 C^{\prime}\left(2+\left(\delta-\frac{1}{2}\right)^{-1}\right)(a-h)^{(1-\delta)} \\
& -C(a-h)^{(\delta-1)}-6 C^{\prime}(a-h)^{-\frac{3}{2}}-\frac{1}{2} C_{1}(z)-R(a, h, s) \\
\geq & C a^{\delta}-C h a^{\delta-1}-4 C^{\prime}\left(2+\left(\delta-\frac{1}{2}\right)^{-1}\right) a^{(1-\delta)} \\
& -C a^{(\delta-1)}-6 C^{\prime} a^{-\frac{3}{2}}-\frac{1}{2} C-R(a, h, s) \\
\geq & C a^{\delta}-\left(3,9 \cdot C C_{0}^{-2 / 3}+7,8 \cdot C_{0}^{1 / 3}\right) \cdot a^{1 / 3(1+\delta)} \\
& -3,2 \cdot C_{0}^{-1 / 3} a^{1 / 3(2-\delta)}-6.7 \cdot C_{0}^{-2 / 3} a^{1 / 6(5-4 \delta)} \\
& -\left(\log C_{0}+\frac{1}{2} \log a+2 \cdot \nu(r) \rho+19\right) \cdot a^{1 / 2} \\
& -4 C^{\prime}\left(2+\left(\delta-\frac{1}{2}\right)^{-1}\right) a^{(1-\delta)}-1,5 \cdot C-6 C^{\prime} .
\end{aligned}
$$

Die Bedingungen an $a, h_{0}$ und $s_{0}$ sind die gleichen wie oben. Damit ist Theorem $\mathrm{D}$ bewiesen.

\subsection{Anwendungen}

In diesem Kapitel sollen zwei Anwendungen von Theorem C und D gegeben werden. Die erste ist als einfache Folgerung aus Theorem $\mathrm{C}$ ein Test auf die Existenz eines zweiten kleinen Eigenwertes für cofinite Fuchssche Gruppen. Als zweite Anwendung werden Theorem C und D dazu benutzt, den Konvergenzexponenten und die erste Eigenfunktion einer geometrisch endlichen Gruppe über deren Gitterpunktzählfunktion zu approximieren.

\subsubsection{Ein Test auf die Existenz eines zweiten kleinen Eigenwertes}

Der kleinste Eigenwert einer cofiniten Fuchsschen Gruppe ist Null und die zugehörige normierte Eigenfunktion $\phi$ ist konstant

$$
\phi(z)=\frac{1}{\sqrt{\operatorname{area}(D / G)}} .
$$

Für cofinite Fuchssche Gruppen folgt aus Theorem C also: 
Korollar B. Es sei $G$ eine cofinite Fuchssche Gruppe mit einem Fundamentalbereich vom Flächeninhalt $A$. Es sei $z \in D$ und

$$
\begin{gathered}
r:=\operatorname{inj}(z)>0 \\
\nu(r):= \begin{cases}\frac{5}{4} & \text { falls } \sinh ^{-2} \frac{r}{2}<\frac{5}{4} \\
\sinh ^{-2} \frac{r}{2} & \text { falls } \sinh ^{-2} \frac{r}{2} \geq \frac{5}{4}\end{cases} \\
X_{0}:=\sqrt{\nu(r)-1 / 4} .
\end{gathered}
$$

Gibt es nun ein $a \in \mathbb{R}$ mit

$$
\begin{gathered}
a>\max \left\{6,0,6 \cdot C_{0}^{2 / 3}\right\}, \\
a>\left(C_{0}^{-2 / 3} \cdot \max \left\{7,8,1,6 \cdot X_{0}\right\}\right)^{2},
\end{gathered}
$$

so daß mit $\rho>0$ definiert durch $a=\cosh \rho$ gilt, daß

$$
\begin{aligned}
N(a ; z, z)> & \frac{2 \pi}{A} a+15 \cdot\left(\frac{2 \pi}{A}\right)^{1 / 3} \cdot a^{2 / 3} \\
& +18 \cdot\left(\frac{2 \pi}{A}\right)^{-1 / 3} a^{1 / 3}+19 \cdot\left(\frac{2 \pi}{A}\right)^{-2 / 3} a^{1 / 6} \\
& +\left(1,5 \cdot \log \frac{2 \pi}{A}+0,8 \cdot \log a+3 \cdot \nu(r) \rho+27\right) \cdot a^{1 / 2}+\frac{3}{A},
\end{aligned}
$$

dann hat der Laplace-Beltrami Operator auf $D / G$ neben dem Eigenwert Null einen weiteren Eigenwert $\lambda_{1}$ mit $0<\lambda_{1}<\frac{1}{4}$.

Eine Klasse von Gruppen, für die ein solcher Test Anwendung finden könnte, sind die Fermat Gruppen. Sie sind Untergruppen von

$$
\Gamma(2):=\left\{\left(\begin{array}{ll}
a & b \\
c & d
\end{array}\right) \in \operatorname{SL}(2, \mathbb{Z}):\left(\begin{array}{ll}
a & b \\
c & d
\end{array}\right) \equiv\left(\begin{array}{ll}
1 & 0 \\
0 & 1
\end{array}\right) \quad \bmod 2\right\} .
$$

$\Gamma(2)$ wird frei erzeugt von

$$
A=\left(\begin{array}{ll}
1 & 2 \\
0 & 1
\end{array}\right) \quad \text { und } \quad B=\left(\begin{array}{cc}
1 & 0 \\
-2 & 1
\end{array}\right)
$$

Die Fermat Gruppe der Ordnung $N$ ist durch

$$
\Phi(N):=\left\{\gamma=A^{p_{1}} B^{q_{1}} A^{p_{2}} B^{q_{2}} \cdots A^{p_{k}} B^{q_{k}}: \sum p_{i} \equiv 0 \equiv \sum q_{i} \bmod N\right\}
$$

definiert. Sie werden in [34] und [32] untersucht. Die Gitterpunktzählfunktion wird in diesen beiden Veröffentlichungen mit einem Programm numerisch untersucht, welches unserem, zu diesem Zwecke geschriebenem Programm, sehr ähnlich ist (siehe Appendix C). In [34] wird von Phillips und Sarnak aufgrund der gewonnenen Daten vermutet, daß es für $N \leq 8$ keine Eigenwerte echt größer als Null und kleiner als 1/4 gibt, wohingegen 
die Fermat Gruppen mit $N \geq 9$ stets solche Eigenwerte besitzen. Die Abbildungen auf Seite 77 und 78 zeigten die mit einem Computerprogramm (siehe Appendix C) berechneten Werte von

$$
d(a ; i, i ; N):=N(a ; i, i ; N)-\frac{1}{N^{2}} \cdot a
$$

für $a=10^{7} \cdot k$ mit $k \in\{1, \ldots, 10000\}$ jeweils für $N=1,7,8,9$. Die letzte Abbildung zeigt das deutliche Anwachsen von $d(a ; i, i ; 9)$ mit $a$, welches zu der Vermutung führt, daß es einen weiteren $L^{2}$-Eigenwert gibt. Eine lineare Regression der Werte $(\log d(a ; i, i ; 9), \log a)$ deutet auf etwas wie

$$
d(a ; i, i ; 9) \sim 0,06 \cdot a^{0.626}
$$

hin. Da der Exponent kleiner als $2 / 3$ ist, liegt $\Phi(9)$ damit anscheinend außerhalb der Möglichkeiten unseres Tests, denn der Fehlerterm in Korolar B ist von der Ordnung $a^{2 / 3}$. Für $N=13$ lassen die mit dem Computer gewonnenen Daten

$$
d(a ; i, i ; 13) \sim 0.06 \cdot a^{0.754}
$$

vermuten. Unter dieser Annahme kann man abschätzen, daß man die Zählfunktion für etwa $a=5 \cdot 10^{19}$ bestimmten müßte, wollte man mit Korolar B zu einem positiven Ergebnis kommen. Das liegt weit außerhalb unserer Möglichkeiten.

\subsubsection{Approximation des Konvergenzexponenten und der ersten Eigenfunktion}

In [30] formuliert Patterson das Problem, den Konvergenzexponenten einer geometrisch endlichen Fuchsschen Gruppe über die Gitterpunktzählfunktion zu vorgegebener Genauigkeit zu approximieren.

Aus Theorem B folgt für eine geometrisch endliche Fuchssche Gruppe mit Konvergenzexponenten $\delta>1 / 2$ und Eigenfunktion $\phi$ zum kleinsten Eigenwert $\delta(1-\delta)$, daß

$$
\log N(a ; z, w) \sim \log C+\delta \log a
$$

mit

$$
C:=2 \sqrt{\pi} \frac{\Gamma(\delta-1 / 2)}{\Gamma(1+\delta)} \phi(z) \phi(w) .
$$

Theorem C und D liefern nun für die Gerade

$$
f(x)=\log C+\delta x
$$

$(x=\log a)$ zwei Konstanten $B_{u}(a)$ und $B_{o}(a)$ mit

$$
B_{u}(a) \leq f(\log a) \leq B_{o}(a) .
$$

Hat man solche Konstanten für zwei oder mehr Werte von $a$, dann sind $\delta$ und $C$ bis auf einen bestimmbaren Fehler festgelegt. Die betrachtete Fuchssche Gruppe darf allerdings nur genau einen kleinen Eigenwert besitzen. Außerdem benötigt man eine untere Schranke für $\delta$, die echt größer als $1 / 2$ ist, da ansonsten in Theorem $\mathrm{C}$ und $\mathrm{D}$ auftretende Konstanten nicht nach oben abgeschätzt werden können. 
Korollar C. Es sei $G$ eine geometrisch endliche Fuchssche Gruppe, die nur genau einen Eigenwert kleiner als $1 / 4$ hat. Für den Konvergenzexponenten $\delta$ von $G$ gebe es eine Zahl $\delta_{0}>1 / 2$, sodaß $\delta \geq \delta_{0}$. Die normierte Eigenfunktion zum kleinsten Eigenwert $\delta(1-\delta)$ sei $\phi$. Es sei $z \in D, r:=\operatorname{inj}(z)>0$ und $\nu, X_{0}$ wie in Theorem $C$,

$$
C:=2 \sqrt{\pi} \frac{\Gamma(\delta-1 / 2)}{\Gamma(1+\delta)} \phi^{2}(z) .
$$

Weiter sei

$$
f(x):=\log C+\delta x .
$$

Dann gilt für alle $C_{0}>0$ und mit $\rho>0$ definiert durch $a=\cosh \rho$ und für alle $a \in \mathbb{R}$ mit

$$
\begin{gathered}
a>\max \left\{6,0,6 \cdot C_{0}^{2 / 3}\right\}, \\
a>\left(C_{0}^{-2 / 3} \cdot \max \left\{7,8,1,6 \cdot X_{0}\right\}\right)^{\frac{2}{2 \delta_{0}-1}}
\end{gathered}
$$

und mit

$$
\begin{aligned}
B_{o}(a):= & \log \left\{N(a ; z, z)+7,8 \cdot C_{0}^{1 / 3} a^{2 / 3}+3,2 \cdot C_{0}^{-1 / 3} a^{1 / 3\left(2-\delta_{0}\right)}\right. \\
+ & \left.6,7 \cdot C_{0}^{-2 / 3} a^{1 / 6\left(5-4 \delta_{0}\right)}+\left(\log C_{0}+\frac{1}{2} \log a+2 \cdot \nu(r) \rho+19\right) \cdot a^{1 / 2}\right\} \\
- & \log \left\{1-3,9 \cdot C_{0}^{-2 / 3} a^{1 / 3\left(1-2 \delta_{0}\right)}-\frac{2}{\pi}\left(2+\left(\delta_{0}-\frac{1}{2}\right)^{-1}\right) a^{\left(1-2 \delta_{0}\right)}\right. \\
\left.-1,5 a^{-\delta_{0}}-\frac{3}{\pi} a^{-\delta_{0}}\right\} & \\
B_{u}(a):= & \log \left\{N(a ; z, z)-11,1 \cdot C_{0}^{1 / 3} a^{2 / 3}-18 \cdot C_{0}^{-1 / 3} a^{1 / 3\left(2-\delta_{0}\right)}\right. \\
& -19 \cdot C_{0}^{-2 / 3} a^{1 / 6\left(5-4 \delta_{0}\right)} \\
& \left.-\left(1,5 \cdot \log C_{0}+0,8 \cdot \log a+3 \cdot \nu(r) \rho+27\right) \cdot a^{1 / 2}\right\} \\
& -\log \left(1+3,9 \cdot C_{0}^{-2 / 3} a^{1 / 3\left(1-2 \delta_{0}\right)}+\frac{3}{2 \pi} a^{-\delta_{0}}\right)
\end{aligned}
$$

$d a \beta$

$$
B_{u}(a) \leq f(\log a) \leq B_{o}(a) .
$$

Erfüllen $a_{1}$ und $a_{2}$ mit $a_{1}<a_{2}$ die oben an a gestellten Bedingungen, so gilt für den Konvergenzexponenten $\delta$ von $G, d a \beta$

$$
\frac{B_{u}\left(a_{2}\right)-B_{o}\left(a_{1}\right)}{\log a_{2}-\log a_{1}} \leq \delta \leq \frac{B_{o}\left(a_{2}\right)-B_{u}\left(a_{1}\right)}{\log a_{2}-\log a_{1}}
$$

und für $C$ wie oben definiert, daß

$$
\frac{\log \left(a_{2}\right) B_{u}\left(a_{1}\right)-\log \left(a_{1}\right) B_{o}\left(a_{2}\right)}{\log a_{2}-\log a_{1}} \leq C \leq \frac{\log \left(a_{2}\right) B_{o}\left(a_{1}\right)-\log \left(a_{1}\right) B_{u}\left(a_{2}\right)}{\log a_{2}-\log a_{1}} .
$$


Die Familie der Heckegruppen besteht aus den durch die beiden in der oberen Halbebene operierenden Isometrien

$$
z \mapsto z+\mu, z \mapsto-\frac{1}{z}
$$

frei erzeugten Gruppen, welche im weiteren mit $\Gamma_{\mu}$ bezeichnet werden sollen. Für $\mu \geq 2$ ist $\Gamma_{\mu}$ eine Fuchssche Gruppe. In [33] beweisen Phillips und Sarnak in Theorem 6.1. .

Theorem (Phillips und Sarnak). Für $\mu>2$ hat die Heckegruppe $\Gamma_{\mu}$ genau einen diskreten Eigenwert $\lambda_{0}(\mu)$. Es gilt

$$
\lambda_{0}(2)=0 \text { und } \lim _{\mu \rightarrow \infty} \lambda_{0}(\mu)=1 / 4 .
$$

Als Funktion von $\mu$ wächst $\lambda_{0}$ streng monoton.

Die Tatsache (3.4.1) wurde bereits von Beardon [2] mit rein geometrischen Mitteln bewiesen. Die Monotonie wurde zuerst von Elstrod [7] und unabhängig von Elstrod auch von Patterson [27] bewiesen. In [2] beweist Beardon insbesondere das folgende Theorem:

Theorem 3.4.1 (Beardon). Es seien s und $t$ die beiden eindeutigen reellen Zahlen mit

$$
\sum_{n=1}^{\infty}(n \mu+1)^{-2 s}=\frac{1}{2}=\sum_{n=1}^{\infty}(n \mu-1)^{-2 t}
$$

und $\delta(\mu)$ der Konvergenzradius von $\Gamma_{\mu}$. Dann gilt $\frac{1}{2}<s \leq \delta(\mu) \leq t$.

Hiermit schließt Beardon auf (3.4.1). Wir wollen dieses Theorem benutzen, um eine untere Schranke für den Konvergenzexponenten $\delta(\mu)$ von $\Gamma_{\mu}$ zu erhalten. Gibt es für ein $\delta_{0}>1 / 2$ ein $N \in \mathbb{N}$ mit

$$
\sum_{n=1}^{N}(n \mu+1)^{-2 \delta_{0}} \geq \frac{1}{2},
$$

dann gilt nach Theorem 3.4.1, daß $\delta \geq \delta_{0}$ ist. Die Heckegruppen $\Gamma_{\mu}$ haben nach Theorem 3.4.2 nur genau einen Eigenwert kleiner als 1/4, wir können also mit Hilfe von Korollar $\mathrm{C}$ durch Auszählen von Gitterpunkten den Konvergenzexponent $\delta(\mu)$ und

$$
2 \sqrt{\pi} \frac{\Gamma(\delta-1 / 2)}{\Gamma(1+\delta)} \phi^{2}(z),
$$

wobei $\phi$ die normierte Eigenfunktion zum kleinsten Eigenwert $\delta(\mu)(1-\delta(\mu))$ ist, mit vorgegebener Genauigkeit approximieren. Mit dem im Appendix $\mathrm{C}$ angegebenen Computerprogramm kann der Wert der Gitterpunktzählfunktion $N(a ; 2 i, 2 i)$ für die Heckegruppe $\Gamma_{\mu}$ bestimmt werden.

Als ein Beispiel soll $\Gamma_{2,4}$ betrachtet werden. Wie man leicht mit einem geeigneten Computerprogramm (z.B. Mathematica) nachrechnen kann, gilt

$$
\sum_{n=1}^{1000}(2,4 \cdot n+1)^{-2 \cdot 0,76}=0,5009 \cdots .
$$


Es folgt also $\delta(3) \geq \delta_{0}:=0,76$. Die obere Schranke, die Theorem 3.4.1 liefert, ist größer als Eins und damit ohne Bedeutung, denn für Fuchssche Gruppen ist der Konvergenzexponent ja stets kleiner oder gleich Eins. Mit dem Programm aus Appendix C wurde

$$
\begin{aligned}
& N\left(10^{12} ; 2 i, 2 i\right)=23.383 .324 .507 \\
& N\left(10^{14} ; 2 i, 2 i\right)=1.097 .048 .219 .243
\end{aligned}
$$

ermittelt. Es wurde auf einer SGI O2 gerechnet. Die Rechenzeit betrug etwa eine Woche. Aus Korollar C folgt nun mit $\operatorname{inj}(z)>\log 2$, daß

$$
0,80 \leq \delta(2,4) \leq 0,87 .
$$

In [23] gibt McMullen einen Algorithmus an, den er unter anderem auch zur Berechnung des Konvergenzexponenten der Heckegruppen heranzieht. Er schätzt insbesondere auch die Genauigkeit seiner Berechnungen ab (für eine Diskusion siehe Abschnitt 1.3). 


\section{A. Die Legendrefunktionen}

Die Legendresche Differentialgleichung ist durch

$$
\left(t^{2}-1\right) f^{\prime \prime}(t)+2 t f^{\prime}(t)+\lambda f(t)=0, \quad \lambda \in \mathbb{R}
$$

definiert. Sie besitzt genau bei +1 und -1 schwache Singularitäten, und es gibt deshalb genau eine Lösung $F_{\lambda}$ von A.0.1 mit $F_{\lambda} \in C^{2}[-1, \infty)$ und $F_{\lambda}(1)=1$.

Lemma A.0.2. Ist $G \in C^{2}(1, \infty)$ eine Lösung von A.0.1 mit

$$
\lim _{t \searrow 1} G(t)=\alpha,
$$

so ist

$$
G=\alpha F_{\lambda}
$$

Einen Beweis findet man in [13] S. 217.

Unsere Abschätzungen von $F_{\lambda}$ gewinnen wir aus den folgenden Integraldarstellungen. Der Einfachheit halber definieren wir

$$
\kappa=\kappa(\lambda):=\left|\lambda-\frac{1}{4}\right|^{1 / 2} .
$$

Lemma A.0.3. Für $\tau>0,0 \leq \lambda \leq \frac{1}{4}$ gilt

$$
F_{\lambda}(\cosh \tau)=\frac{\sqrt{2}}{\pi} \int_{0}^{\tau} \frac{\cosh (\kappa(\lambda) u)}{(\cosh \tau-\cosh u)^{1 / 2}} d u .
$$

Für $\tau>0, \lambda>\frac{1}{4}$ gilt

$$
\begin{gathered}
F_{\lambda}(\cosh \tau)=\frac{\sqrt{2}}{\pi} \int_{0}^{\tau} \frac{\cos (\kappa(\lambda) u)}{(\cosh \tau-\cosh u)^{1 / 2}} d u \\
\tanh (\pi \kappa(\lambda)) F_{\lambda}(\cosh \tau)=\frac{\sqrt{2}}{\pi} \int_{\tau}^{\infty} \frac{\sin (\kappa(\lambda) u)}{(\cosh \tau-\cosh u)^{1 / 2}} d u
\end{gathered}
$$

Es bezeichne $a(\lambda)$ die kleinste Nullstelle von $F_{\lambda}$ im Intervall $(1, \infty)$. Nach [10] besitzt $F_{\lambda}$ für $\lambda \leq \frac{1}{4}$ keine Nullstelle in $(1, \infty)$. Wir setzen also

$$
a(\lambda)=+\infty \quad \text { falls } \lambda \leq \frac{1}{4} .
$$


Lemma A.0.4. Es ist

$$
F_{\lambda}(t) \geq 1-\frac{\lambda}{2}(t-1) \quad \text { in }[1, a(\lambda)] .
$$

Insbesondere gilt $a(\lambda) \geq 1+\frac{2}{\lambda}$.

Für einen Beweis siehe [12] S. 258-259.

Lemma A.0.5. Für $\lambda \leq X$ und $1 \leq t \leq a(X)$ gilt

$$
F_{\lambda}(t) \geq F_{X}(t) \geq 0 .
$$

Einen Beweis findet man in [13] auf Seite 221.

\section{A.1. Abschätzungen für kleine Eigenwerte}

Wir wollen jetzt die für den führenden Term wichtige Abschätzung von $\int_{1}^{a} F_{\lambda}(t) d t$ für $0 \leq \lambda \leq \frac{1}{4}$ gewinnen.

Proposition A.1.1. Für $0 \leq \lambda<\frac{1}{4}, a>1$ und mit $a=\cosh \rho$ gilt

$$
\begin{aligned}
& 2 \pi \int_{1}^{a} F_{\lambda}(t) d t \leq C e^{\left(\frac{1}{2}+\kappa\right) \rho}+3 e^{-\frac{1}{2} \rho} \\
& 2 \pi \int_{1}^{a} F_{\lambda}(t) d t \geq C e^{\left(\frac{1}{2}+\kappa\right) \rho}-2\left(2+\kappa^{-1}\right) e^{\left(\frac{1}{2}-\kappa\right) \rho}-C e^{\left(\kappa-\frac{1}{2}\right) \rho}-3 e^{-\frac{3}{2} \rho}
\end{aligned}
$$

mit

$$
C:=\frac{\sqrt{\pi} \Gamma(\kappa)}{\Gamma\left(\frac{3}{2}+\kappa\right)} .
$$

Wegen $2 \cosh \rho-1 \leq \exp \rho \leq 2 \cosh \rho$ folgt sofort

Korollar A.1.2. Für $0 \leq \lambda<\frac{1}{4}, a>1$ und mit $s(\lambda):=\frac{1}{2}+\kappa$ gilt

$$
\begin{aligned}
& 2 \pi \int_{1}^{a} F_{\lambda}(t) d t \leq C a^{s(\lambda)}+3 a^{-\frac{1}{2}} \\
& 2 \pi \int_{1}^{a} F_{\lambda}(t) d t \geq C a^{s(\lambda)}-4\left(2+\kappa^{-1}\right) a^{(1-s(\lambda))}-C a^{(s(\lambda)-1)}-6 a^{-\frac{3}{2}}-\frac{1}{2} C
\end{aligned}
$$

mit

$$
C:=2 \sqrt{\pi} \frac{\Gamma(s(\lambda)-1 / 2)}{\Gamma(1+s(\lambda))} .
$$

Der Beweis ist in drei Lemmata aufgeteilt.

Lemma A.1.3. Für $0 \leq \lambda \leq \frac{1}{4}$ und mit $a=\cosh \xi$ und $X=e^{\xi}$ gilt

$$
2 \pi \int_{1}^{a} F_{\lambda}(t) d t=2 X^{\frac{1}{2}} \int_{1 / X}^{1}(1-y)^{\frac{1}{2}}\left(1-\frac{1}{X^{2} y}\right)^{\frac{1}{2}}\left((X y)^{\kappa(\lambda)}+(X y)^{-\kappa(\lambda)}\right) \frac{d y}{y} .
$$


Beweis. Wir setzen $X=e^{\xi}$ und $X y=e^{u}$. Es ist

$$
\begin{aligned}
2 \pi \int_{1}^{a} F_{\lambda}(t) d t & =2 \pi \int_{0}^{\xi} F_{\lambda}(\cosh \tau) \sinh \tau d \tau \\
& =2 \sqrt{2} \int_{0}^{\xi} \int_{0}^{\tau} \frac{\cosh (\kappa u)}{(\cosh \tau-\cosh u)^{1 / 2}} d u \sinh \tau d \tau \\
& =2 \sqrt{2} \int_{0}^{\xi} \cosh (\kappa u) \int_{u}^{\xi} \frac{\sinh \tau}{(\cosh \tau-\cosh u)^{1 / 2}} d \tau d u \\
& =4 \sqrt{2} \int_{0}^{\xi} \cosh (\kappa u)(\cosh \xi-\cosh u)^{\frac{1}{2}} d u \\
& =2 \int_{0}^{\xi}\left(e^{\kappa u}-e^{-\kappa u}\right)\left(e^{\xi}-e^{-\xi}-e^{u}-e^{-u}\right)^{\frac{1}{2}} d u \\
& =2 X^{\frac{1}{2}} \int_{1 / X}^{1}(1-y)^{\frac{1}{2}}\left(1-\frac{1}{X^{2} y}\right)^{\frac{1}{2}}\left((X y)^{\kappa}+(X y)^{-\kappa}\right) \frac{d y}{y}
\end{aligned}
$$

Lemma A.1.4. Für $\kappa \in\left(0, \frac{1}{2}\right]$ und $X>1$ gilt

$$
\begin{aligned}
& \int_{1 / X}^{1}(1-y)^{\frac{1}{2}} y^{\kappa} \frac{d y}{y} \leq \frac{\Gamma\left(\frac{3}{2}\right) \Gamma(\kappa)}{\Gamma\left(\frac{3}{2}+\kappa\right)}-\kappa^{-1} X^{-\kappa}+X^{-\kappa-1}, \\
& \int_{1 / X}^{1}(1-y)^{\frac{1}{2}} y^{\kappa} \frac{d y}{y} \geq \frac{\Gamma\left(\frac{3}{2}\right) \Gamma(\kappa)}{\Gamma\left(\frac{3}{2}+\kappa\right)}-\kappa^{-1} X^{-\kappa} .
\end{aligned}
$$

Beweis. Es ist

$$
\int_{1 / X}^{1}(1-y)^{\frac{1}{2}} y^{\kappa} \frac{d y}{y}=\frac{\Gamma\left(\frac{3}{2}\right) \Gamma(\kappa)}{\Gamma\left(\frac{3}{2}+\kappa\right)}-\int_{0}^{1 / X}(1-y)^{\frac{1}{2}} y^{\kappa} \frac{d y}{y}
$$

und

$$
\int_{0}^{1 / x}(1-y)^{\frac{1}{2}} y^{\kappa} \frac{d y}{y}=\kappa^{-1} X^{-\kappa}+\int_{0}^{1 / x}\left((1-y)^{\frac{1}{2}}-1\right) y^{\kappa} \frac{d y}{y} .
$$

Mit $0 \leq 1-(1-y)^{\frac{1}{2}} \leq y$ folgt

$$
-X^{-\kappa-1} \leq \int_{0}^{1 / X}(1-y)^{\frac{1}{2}} y^{\kappa} \frac{d y}{y}-\kappa^{-1} X^{-\kappa} \leq 0 .
$$

Setzt man das in A.1.1 ein, so ist die Behauptung bewiesen.

Lemma A.1.5. Für $\kappa \in\left(0, \frac{1}{2}\right]$ und $X>1$ gilt

$$
\begin{aligned}
& \int_{1 / X}^{1}(1-y)^{\frac{1}{2}} y^{-\kappa} \frac{d y}{y} \leq \kappa^{-1} X^{\kappa}+\frac{1}{2} X^{\kappa-1}, \\
& \int_{1 / X}^{1}(1-y)^{\frac{1}{2}} y^{-\kappa} \frac{d y}{y} \geq \kappa^{-1} X^{\kappa}-\left(2+\kappa^{-1}\right) .
\end{aligned}
$$


Beweis. Es gilt

$$
A(X):=\int_{1 / X}^{1}(1-y)^{\frac{1}{2}} y^{-\kappa} \frac{d y}{y}-\frac{X^{\kappa}}{\kappa}=\int_{1 / X}^{1}\left((1-y)^{\frac{1}{2}}-1\right) y^{-\kappa} \frac{d y}{y}-\kappa^{-1} .
$$

Wir benutzen $\frac{1}{2} y \leq 1-(1-y)^{\frac{1}{2}} \leq y$ und erhalten

$$
\begin{aligned}
A(X) & \leq \frac{1}{2} \int_{1 / X}^{1} y^{-\kappa} d y-\kappa^{-1}=-\kappa^{-1}+\frac{1}{2-2 \kappa} X^{\kappa-1}-\frac{1}{2-2 \kappa} \\
& \leq \frac{1}{2} X^{\kappa-1}
\end{aligned}
$$

und

$$
\begin{aligned}
A(X) & \geq \int_{1 / X}^{1} y^{-\kappa} d y-\kappa^{-1}=-\kappa^{-1}+\frac{1}{1-\kappa} X^{\kappa-1}-\frac{1}{1-\kappa} \\
& \geq-\kappa^{-1}-2 .
\end{aligned}
$$

Wir können jetzt Proposition A.1.1 beweisen. Es ist

$$
\begin{aligned}
& \int_{1 / X}^{1}\left(1-\frac{1}{X^{2} y}\right) f(y) \frac{d y}{y} \leq \int_{1 / X}^{1} f(y) \frac{d y}{y} \text { und } \\
& \int_{1 / X}^{1}\left(1-\frac{1}{X^{2} y}\right) f(y) \frac{d y}{y} \geq \int_{1 / X}^{1} f(y) \frac{d y}{y}-\frac{1}{X} \int_{1 / X}^{1} f(y) \frac{d y}{y},
\end{aligned}
$$

mit $f(y):=(1-y)^{\frac{1}{2}}\left((X y)^{\kappa}+(X y)^{-\kappa}\right)$ und Lemma A.1.3, A.1.4 und A.1.5 folgt

$$
\begin{aligned}
2 \pi \int_{1}^{a} F_{\lambda}(t) d t & \leq 2 X^{\frac{1}{2}+\kappa} \int_{1 / X}^{1}(1-y)^{\frac{1}{2}} y^{\kappa} \frac{d y}{y}+2 X^{\frac{1}{2}+\kappa} \int_{1 / X}^{1}(1-y)^{\frac{1}{2}} y^{-\kappa} \frac{d y}{y} \\
& \leq \sqrt{\pi} \frac{\Gamma(\kappa)}{\Gamma\left(\frac{3}{2}+\kappa\right)} X^{\frac{1}{2}+\kappa}-2 \kappa^{-1} X^{\frac{1}{2}}+2 X^{-\frac{1}{2}}+2 \kappa^{-1} X^{\frac{1}{2}}+X^{-\frac{1}{2}} \\
& =\sqrt{\pi} \frac{\Gamma(\kappa)}{\Gamma\left(\frac{3}{2}+\kappa\right)} X^{\frac{1}{2}+\kappa}+3 X^{-\frac{1}{2}} .
\end{aligned}
$$

Außerdem gilt

$$
\begin{aligned}
2 \pi \int_{1}^{a} F_{\lambda}(t) d t \geq & \sqrt{\pi} \frac{\Gamma(\kappa)}{\Gamma\left(\frac{3}{2}+\kappa\right)} X^{\frac{1}{2}+\kappa}-2 \kappa^{-1} X^{\frac{1}{2}}+2 \kappa^{-1} X^{\frac{1}{2}} \\
& -2\left(2+\kappa^{-1}\right) X^{\frac{1}{2}-\kappa}-\sqrt{\pi} \frac{\Gamma(\kappa)}{\Gamma\left(\frac{3}{2}+\kappa\right)} X^{\kappa-\frac{1}{2}}-3 X^{-\frac{3}{2}} \\
\geq & \sqrt{\pi} \frac{\Gamma(\kappa)}{\Gamma\left(\frac{3}{2}+\kappa\right)} X^{\frac{1}{2}+\kappa}-2\left(2+\kappa^{-1}\right) X^{\frac{1}{2}-\kappa} \\
& -\sqrt{\pi} \frac{\Gamma(\kappa)}{\Gamma\left(\frac{3}{2}+\kappa\right)} X^{\kappa-\frac{1}{2}}-3 X^{-\frac{3}{2}}
\end{aligned}
$$


Damit ist Proposition A.1.1 bewiesen. Für $\lambda=0$ ergibt sich

Lemma A.1.6. Für $a \geq 1$ gilt

$$
\int_{1}^{a} F_{0}(t) d t=a-1
$$

\section{A.2. Abschätzungen für große Eigenwerte}

Die folgenden Abschätzungen sind wichtig für die Behandlung des kontinuierlichen Spektrums.

Lemma A.2.1. Für $a>1, a=\cosh \rho$ gilt die Abschätzung

$$
2 \pi\left|\int_{1}^{a} F_{1 / 4}(t) d t\right| \leq 8 \rho a^{1 / 2} .
$$

Beweis. Nach Proposition A.1.3 ist mit $a=\cosh \rho$ und $X=e^{\rho}$

$$
2 \pi \int_{1}^{a} F_{1 / 4}(t) d t=4 X^{\frac{1}{2}} \int_{1 / X}^{1}(1-y)^{\frac{1}{2}}\left(1-\frac{1}{X^{2} y}\right)^{\frac{1}{2}} \frac{d y}{y} .
$$

Durch partielle Integration folgt

$$
\begin{aligned}
2 \pi \int_{1}^{a} F_{1 / 4}(t) d t & \leq 4 X^{\frac{1}{2}} \int_{1 / X}^{1}(1-y)^{\frac{1}{2}} d \log y \\
& =4 X^{\frac{1}{2}}\left(\left[(1-y)^{\frac{1}{2}} \log y\right]_{1 / X}^{1}+\frac{1}{2} \int_{1 / X}^{1} \log y(1-y)^{\frac{1}{2}} d y\right) \\
& \leq 4 X^{\frac{1}{2}} \log X \\
& \leq 8 \rho a^{1 / 2}
\end{aligned}
$$

Proposition A.2.2. Es sei $\lambda>\frac{1}{4}$ und

$$
G_{\lambda}(x):=\int_{1}^{x} F_{\lambda}(y) d y .
$$

Für $x>1$ gilt

$$
\left|G_{\lambda}(x)\right| \leq \frac{2 \sqrt{2}}{\pi} \frac{x^{1 / 2}}{\kappa}
$$

und für $x \geq 3$ gilt

$$
\begin{aligned}
& \left|G_{\lambda}(x)\right| \leq \alpha \frac{x^{1 / 2}}{\kappa^{3 / 2}}+\gamma \frac{x^{1 / 2}}{\kappa^{2}}, \\
& \alpha:=\sqrt{\frac{2}{\pi}}, \quad \gamma:=\frac{289}{144} \frac{\sqrt{2}}{\pi} .
\end{aligned}
$$


Eine Beweis findet sich in [15] S. 152-156. Er ist sehr gut lesbar und benutzt die Integraldarstellung (A.0.3). Für große $\lambda$ wird die folgende Abschätzung wichtig sein.

Proposition A.2.3. Für $\lambda>\frac{1}{4}$ und $x>1$ gilt

$$
\begin{gathered}
\tanh \pi \kappa\left|F_{\lambda}(x)\right| \leq \frac{\alpha}{\kappa^{1 / 2}}\left(x^{2}-1\right)^{-1 / 4}+\frac{\beta}{\kappa} x^{1 / 2}\left(x^{2}-1\right)^{-1 / 2}, \\
\alpha:=\sqrt{\frac{2}{\pi}}, \quad \beta:=\frac{19}{18} \frac{\sqrt{2}}{\pi} .
\end{gathered}
$$

Für einen Beweis siehe [15] S. 150-152. Hier wird die Integraldarstellung (A.0.4) benutzt. 


\section{B. Einige Abschätzungen}

Die im Abschnitt 3.3 benötigten Rechnungen sind der Übersichtlichkeit halber in diesem Appendix zusammengefaßt. Wir müssen

$$
H\left(a, h_{0}, s_{0}\right):=C h_{0} a^{\delta-1}+\left(a+h_{0}\right)^{\frac{1}{2}} \widehat{R}\left(a, h_{0}, s_{0}\right)
$$

mit

$$
\begin{aligned}
\widehat{R}(a, h, s):= & \frac{15 \alpha a}{h s^{1 / 2}}+\frac{9 \beta a}{h s}+\frac{20 \alpha}{s^{1 / 2}}+\frac{18 \beta}{s} \\
& +6 \alpha s^{1 / 2}+3 \gamma \log s+10^{-2} s^{-1}+\frac{6}{\pi} \nu(r) \rho+\frac{15 \gamma}{8}-\frac{33 \alpha}{8}
\end{aligned}
$$

und

$$
\begin{aligned}
s_{0} & :=\frac{1}{2} C_{0}^{2 / 3}\left(\frac{25 \pi}{36}\right)^{1 / 3} a^{1 / 3(2 \delta-1)}, \\
h_{0} & :=5 C_{0}^{-2 / 3}\left(\frac{36}{25 \pi}\right)^{1 / 3} a^{2 / 3(2-\delta)}
\end{aligned}
$$

abschätzen. Es gilt:

$$
C h_{0} a^{\delta-1} \leq 3,9 \cdot C C_{0}^{-2 / 3} a^{1 / 3(1+\delta)} .
$$

Da vorausgesetzt ist, daß $h_{0}<a$ gilt

$$
\left(a+h_{0}\right)^{1 / 2} \leq \sqrt{2} a^{1 / 2} .
$$

Durch einfaches Einsetzen erhält man weiter

$$
\begin{aligned}
& 15 \alpha a h_{0}^{-1} s_{0}^{-1 / 2}= 3 \sqrt{2} \alpha\left(\frac{25 \pi}{36}\right)^{1 / 6} C_{0}^{1 / 3} a^{1 / 3 \delta-1 / 6} \\
& \leq 3,9 \cdot C_{0}^{1 / 3} a^{1 / 3 \delta-1 / 6} \\
& 9 \beta a h_{0}^{-1} s_{0}^{-1}=\frac{18}{5} \beta \\
& \leq 1,8 \\
& 20 \alpha s_{0}^{-1 / 2}=\frac{20}{\sqrt{2}} \alpha C_{0}^{-1 / 3}\left(\frac{36}{25 \pi}\right)^{1 / 6} a^{-1 / 6(2 \delta-1)} \\
& \leq 12,5 \cdot C_{0}^{-1 / 3} a^{-1 / 6(2 \delta-1)}
\end{aligned}
$$




$$
\begin{aligned}
18 \beta s_{0}^{-1}= & 2 \cdot 18 \beta C_{0}^{-2 / 3}\left(\frac{25 \pi}{36}\right)^{-1 / 3} a^{-1 / 3(2 \delta-1)} \\
\leq & 13,2 \cdot C_{0}^{-2 / 3} a^{-1 / 3(2 \delta-1)}, \\
6 \alpha s_{0}^{1 / 2} & =\frac{6 \alpha}{\sqrt{2}} C_{0}^{1 / 3}\left(\frac{25 \pi}{36}\right)^{1 / 6} a^{1 / 6(2 \delta-1)} \\
& \leq 3.9 C_{0}^{1 / 3} a^{1 / 6(2 \delta-1)}, \\
3 \gamma \log s_{0}= & 3 \gamma \log \left(C_{0}^{2 / 3}\left(\frac{25 \pi}{8 \cdot 36}\right)^{1 / 3} a^{1 / 3(2 \delta-1)}\right) \\
\leq & 2 \gamma \log C_{0}+\gamma \log a \\
\leq & \log C_{0}+\frac{1}{2} \log a, \\
10^{-2} s_{0}^{-1} & =\frac{1}{200} C_{0}^{-2 / 3}\left(\frac{25 \pi}{36}\right)^{-1 / 3} a^{-1 / 3(2 \delta-1)} \\
\leq & 4 \cdot 10^{-3} C_{0}^{-2 / 3} a^{-1 / 3(2 \delta-1)} .
\end{aligned}
$$

Und es gilt also

$$
\begin{aligned}
H\left(a, h_{0}, s_{0}\right) \leq & 3,9 \cdot C C_{0}^{-2 / 3} a^{1 / 3(1+\delta)} \\
& +\sqrt{2} \cdot a^{1 / 2} \cdot\left\{3,9 \cdot C_{0}^{1 / 3} a^{1 / 3 \delta-1 / 6}+1,8+12,5 \cdot C_{0}^{-1 / 3} a^{-1 / 6(2 \delta-1)}\right. \\
& +13,2 \cdot C_{0}^{-2 / 3} a^{-1 / 3(2 \delta-1)}+3.9 C_{0}^{1 / 3} a^{1 / 6(2 \delta-1)} \\
& +\log C_{0}+\frac{1}{2} \log a+4 \cdot 10^{-3} C_{0}^{-2 / 3} a^{-1 / 3(2 \delta-1)} \\
& +2 \cdot \nu(r) \rho+16,8\} \\
\leq & 3,9 \cdot C C_{0}^{-2 / 3} a^{1 / 3(1+\delta)} \\
& +\sqrt{2} \cdot\left\{7.8 \cdot C_{0}^{1 / 3} a^{1 / 3(1+\delta)}+12,5 \cdot C_{0}^{-1 / 3} a^{1 / 3(2-\delta)}\right. \\
& \left.+13,3 \cdot C_{0}^{-2 / 3} a^{1 / 6(5-4 \delta)}+\left(\log C_{0}+\frac{1}{2} \log a+2 \cdot \nu(r) \rho+18,6\right) \cdot a^{1 / 2}\right\} \\
\leq & \left(3,9 \cdot C C_{0}^{-2 / 3}+11,1 \cdot C_{0}^{1 / 3}\right) \cdot a^{1 / 3(1+\delta)}+18 \cdot C_{0}^{-1 / 3} a^{1 / 3(2-\delta)} \\
& +19 \cdot C_{0}^{-2 / 3} a^{1 / 6(5-4 \delta)}+\left(1,5 \cdot \log C_{0}+0,8 \cdot \log a+3 \cdot \nu(r) \rho+27\right) \cdot a^{1 / 2} .
\end{aligned}
$$

Für die untere Schranke benötigen wir eine Abschätzung von $R\left(a, h_{0}, s_{0}\right)$. Die Rechnung ist die gleiche wie oben, nur daß der Term $20 \alpha s_{0}^{-1 / 2}$ durch $5 \alpha s_{0}^{-1 / 2}$ und der Term 
$18 \beta s_{0}^{-1}$ durch $9 \beta s_{0}^{-1}$ ersetzt werden muß.Außerdem fällt der Multiplikator $\sqrt{2}$ vor der geschweiften Klammer weg.

$$
\begin{aligned}
C h_{0} a^{\delta-1}+R\left(a, h_{0}, s_{0}\right) \leq & \left(3,9 \cdot C C_{0}^{-2 / 3}+7,8 \cdot C_{0}^{1 / 3}\right) \cdot a^{1 / 3(1+\delta)} \\
& +3,2 \cdot C_{0}^{-1 / 3} a^{1 / 3(2-\delta)}+6.7 \cdot C_{0}^{-2 / 3} a^{1 / 6(5-4 \delta)} \\
& +\left(\log C_{0}+\frac{1}{2} \log a+2 \cdot \nu(r) \rho+19\right) \cdot a^{1 / 2} .
\end{aligned}
$$

Nun sollen noch die Bedingungen an $a, h_{0}, s_{0}$ als Bedingungen an $a$ formuliert werden. Die obigen Abschätzungen sind richtig, wenn

$$
\begin{aligned}
a & >5, \\
h_{0} & \geq 2, \\
h_{0} & <a-3, \\
s_{0} & \geq X_{0} .
\end{aligned}
$$

Die Bedingung $h_{0} \geq 2$ ist sicherlich erfüllt, wenn

$$
a \geq \frac{2}{5} C_{0}^{2 / 3}\left(\frac{36}{25 \pi}\right)^{1 / 3}
$$

oder

$$
a \geq 0.6 \cdot C_{0}^{2 / 3} .
$$

Die Bedingung $h_{0}<a-3$ läßt sich schreiben als

$$
a\left(1-5 C_{0}^{-2 / 3}\left(\frac{36}{25 \pi}\right)^{1 / 3} a^{1 / 3(1-2 \delta)}\right)>3 .
$$

Wir fordern $a>6$. Dann ist (B.0.1) erfüllt, wenn

$$
\left(1-5 C_{0}^{-2 / 3}\left(\frac{36}{25 \pi}\right)^{1 / 3} a^{1 / 3(1-2 \delta)}\right)<\frac{1}{2}
$$

Das ist äquivalent zu

$$
a^{1 / 3(1-2 \delta)} \geq 10 \cdot\left(\frac{36}{25 \pi}\right)^{1 / 3} \cdot C_{0}^{-2 / 3}
$$

was gilt, wenn

$$
a^{1 / 3(1-2 \delta)} \geq 7,8 \cdot C_{0}^{-2 / 3} .
$$

Schließlich gilt $s_{0} \geq X_{0}$, wenn

$$
a^{1 / 3(1-2 \delta)} \geq 2 \cdot\left(\frac{36}{25 \pi}\right)^{1 / 3} \cdot X_{0} C_{0}^{-2 / 3}
$$


oder wenn

$$
a^{1 / 3(1-2 \delta)} \geq 1,6 \cdot X_{0} C_{0}^{-2 / 3} .
$$

Zusammen ergeben sich folgende Forderungen an $a$ :

$$
\begin{gathered}
a>\max \left\{6,0,6 \cdot C_{0}^{2 / 3}\right\}, \\
a>\left(C_{0}^{-2 / 3} \cdot \max \left\{7,8,1,6 \cdot X_{0}\right\}\right)^{\frac{2}{2 \delta-1}} .
\end{gathered}
$$




\section{Das Computerprogramm}

In diesem Appendix wird ein Computerprogramm in C, welches die Gitterpunktzählfunktion für die (erweiterten) Fermat Gruppen auszählt, erläutert und abgedruckt. AuBerdem ist noch ein kurzes C-Programm zum schnellen Auszählen von Gitterpunkten des Gitters $\mathbb{Z}^{2}$ beigefügt.

In der oberen Halbebene betrachten wir die beiden Isometrien

$$
A=\left(\begin{array}{cc}
1 & \mu \\
0 & 1
\end{array}\right) \quad \text { und } \quad B=\left(\begin{array}{cc}
1 & 0 \\
-2 & 1
\end{array}\right), \quad \mu \geq 2
$$

Es sei daran erinnert, daß die Matrix

$$
A=\left(\begin{array}{cc}
a & b \\
c & d
\end{array}\right), \quad a, b, c, d \in \mathbb{R}
$$

die Abbildung

$$
g(z)=\frac{a z+b}{c z+d}
$$

der oberen Halbebene $\mathbb{H}$ in sich repräsentiert, welche eine Isometrie bezüglich der auf $\mathbb{H}$ gegebenen Metrik ist. Die Fermat Gruppe der Ordnung $N$ ist durch

$$
\Phi(N):=\left\{\gamma=A^{p_{1}} B^{q_{1}} A^{p_{2}} B^{q_{2}} \cdots A^{p_{k}} B^{q_{k}}: \sum p_{i} \equiv 0 \equiv \sum q_{i} \bmod N\right\}
$$

mit $\mu=2$ definiert. Sie ist eine Fuchssche Gruppe. Ihr Fundamentalbereich hat den Flächeninhalt $2 \pi N^{2}$. Für Werte von $\mu>2$ erhalten wir Fuchssche Gruppen mit einem Fundamentalbereich von unendlichem Flächeninhalt.

Das unten aufgeführte Programm bestimmt die Werte von $N\left(a_{j} ; i, w\right)$ für eine Auswahl von Radien $a_{j} \leq a$, indem es alle Worte der Form

$$
\gamma=A^{p_{1}} B^{q_{1}} A^{p_{2}} B^{q_{2}} \cdots A^{p_{k}} B^{q_{k}}, \quad p_{i}, q_{i} \in \mathbb{Z}
$$

der Wortlänge nach berechnet, bis es auf ein Wort stößt dessen zugehörige Isometrie $\gamma$ den Punkt $w$ auf einen Punkt $\gamma(w)$ abbildet, der außerhalb des Kreise mit hyperbolischem Radius $\rho$ mit $a=\cosh \rho$ liegt, also bis $\cosh d(i, \gamma(w))>a$. Für jeden Kreisring $A_{j}:=\left\{w \in \mathbb{H}: a_{j}<\cosh d(i, \gamma(w)) \leq a_{j+1}\right\}$ ist ein Zähler vorgesehen. Liegt $\gamma(w)$ in diesem Annulus und ist $\sum p_{i} \equiv 0 \equiv \sum q_{i} \bmod N$, so wird der entsprechende Zähler erhöht. Wir müssen nun noch prüfen ob wir mit unserem Verfahren tatsächlich alle Gitterpunkte innerhalb des vorgegebenen Kreises um $i$ mit Radius $\rho$ durchlaufen. Dies ist der Fall, wenn sich mit jedem Schritt unseres Programms der Abstand zu $i$ vergrößert. 
Liegt der Punkt $w$ in dem Streifen $S:=\{w \in \mathbb{H}-1<\Im w \leq 1\}$, dann wird er unter Anwendung von $A$ auf einen Punkt außerhalb diese Streifens abgebildet, und sein Abstand zu $i$ vergrößert sich also. Die Abbildung $B$ kann auch als

$$
C A C, \quad \text { mit } \quad C=\left(\begin{array}{cc}
0 & -1 \\
1 & 0
\end{array}\right), \quad \mu=2
$$

geschrieben werden. $C$ ist eine Drehung um den Punkt $i$ um 180 Grad und verändert den Abstand zum Punkt $i$ also nicht. Außerdem hat $C$ die Eigenschaft, einen Punkt außerhalb des Streifens $S$ auf einen innerhalb vom $S$ abzubilden. Daraus schließen wir, daß $B$ einen Punkt $w$ außerhalb von $S$ auf einen innerhalb von $S$ wirft, dann wie $A$ diesen Punkt auf einen außerhalb von $S$ abbildet, wobei sich die Distanz zu $i$ vergrößert und schließlich mit $C$ wieder in den Streifen $S$ zurück abbildet. Die Distanz zu $i$ vergrößert sich also bei jedem Schritt des Programms, und wir haben am Ende sicher jedes Element $\gamma$ von $\Phi(1)$ mit $\cosh d(i, \gamma(w)) \leq a$ genau einmal berechnet. Aus Symmetriegründen müssen wir im Fall $\mu=2$ nur die mit $A$ beginnenden Wörter durchgehen und sparen so die Hälfte der Rechenzeit. Da die Rekursionstiefe für große Radien auch sehr groß wird (für $a=10^{11}$ etwa hat man ein Rekursionstiefe von etwa $10^{6}$ ), kommt es schnell zu einem Überlaufen des internen Stacks. Um dieses Problem zu umgehen, wurde das Programm leicht modifiziert. Die lokalen Variablen werden in Arrays verwaltet, und die Rekursion wird mit Hilfe von goto-Befehlen aufgelöst. Das Programm ist dem in [32] und [34] recht ähnlich hat aber den Vorteil, daß nur mit ganzzahligen Matrixeinträgen gerechnet wird und so Rundungsfehler (bis zum endgültigem Bestimmen der Abstandsfunktion) ausgeschlossen sind.

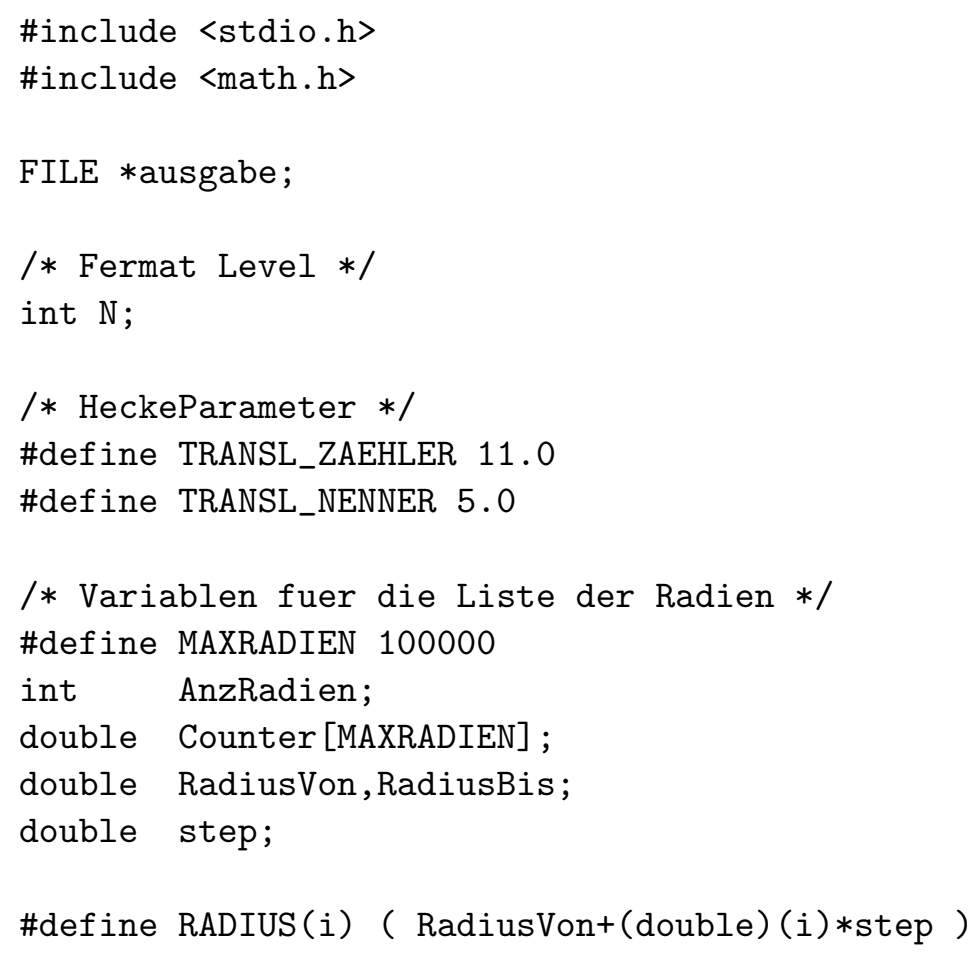




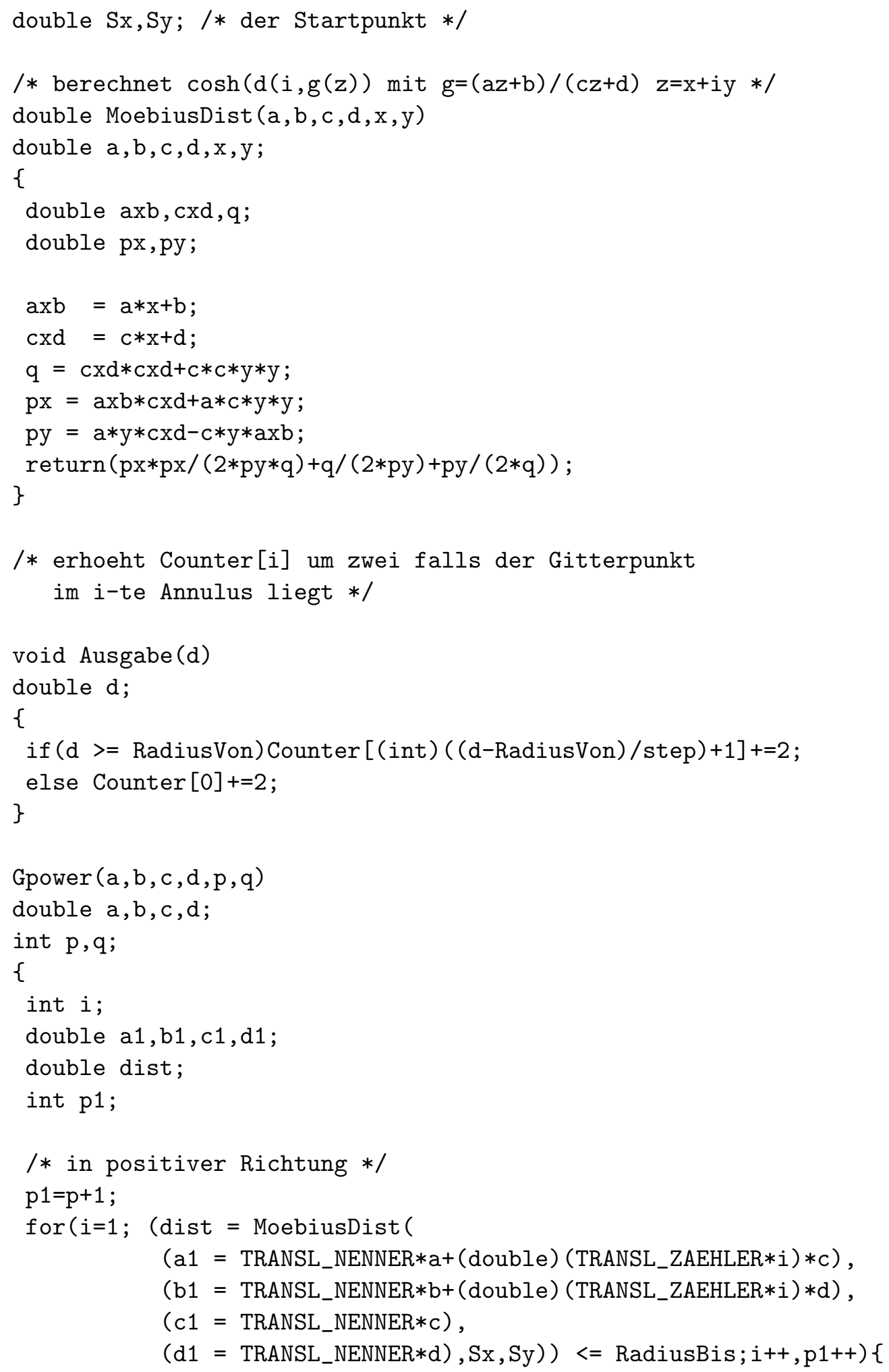




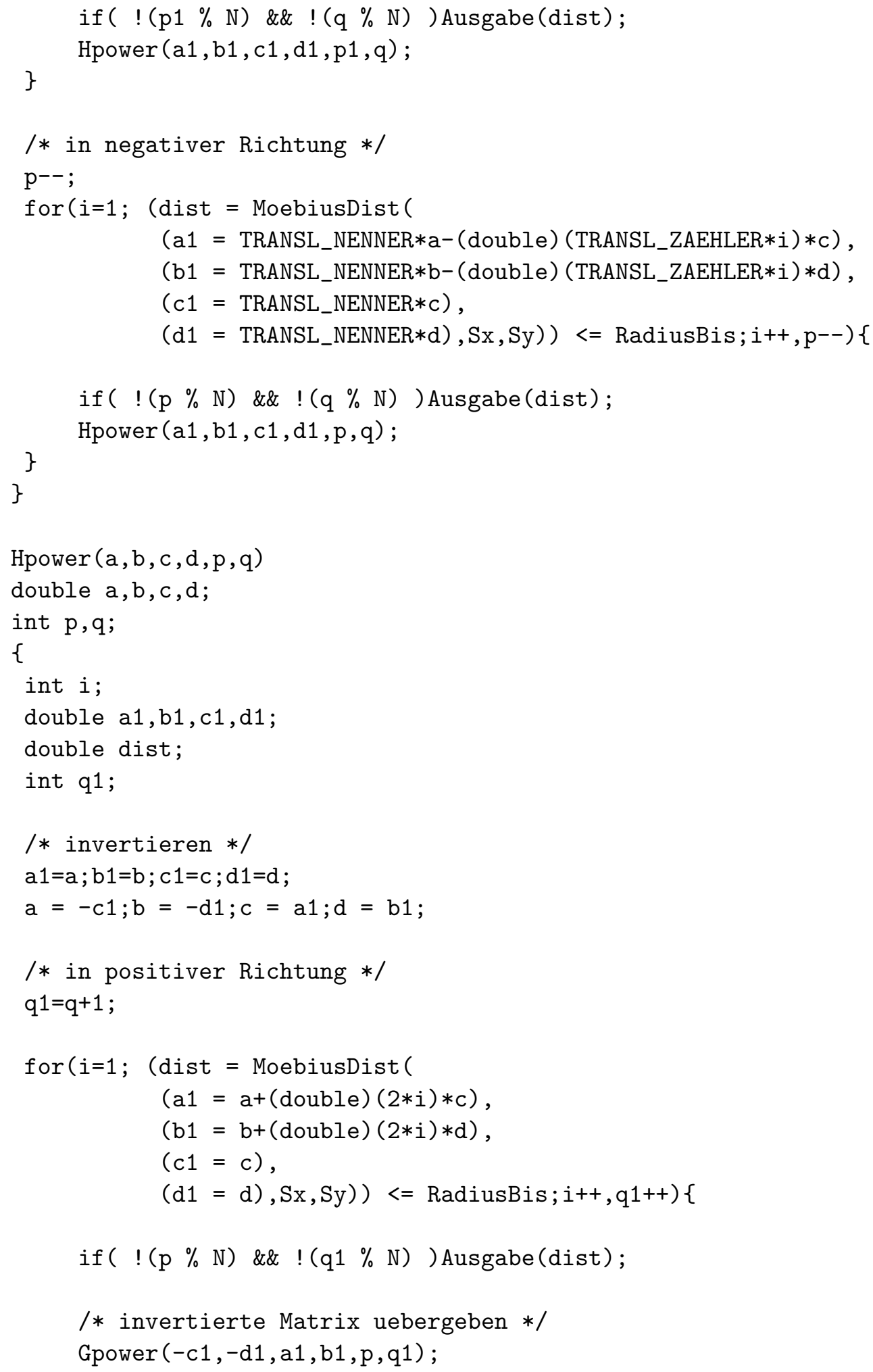




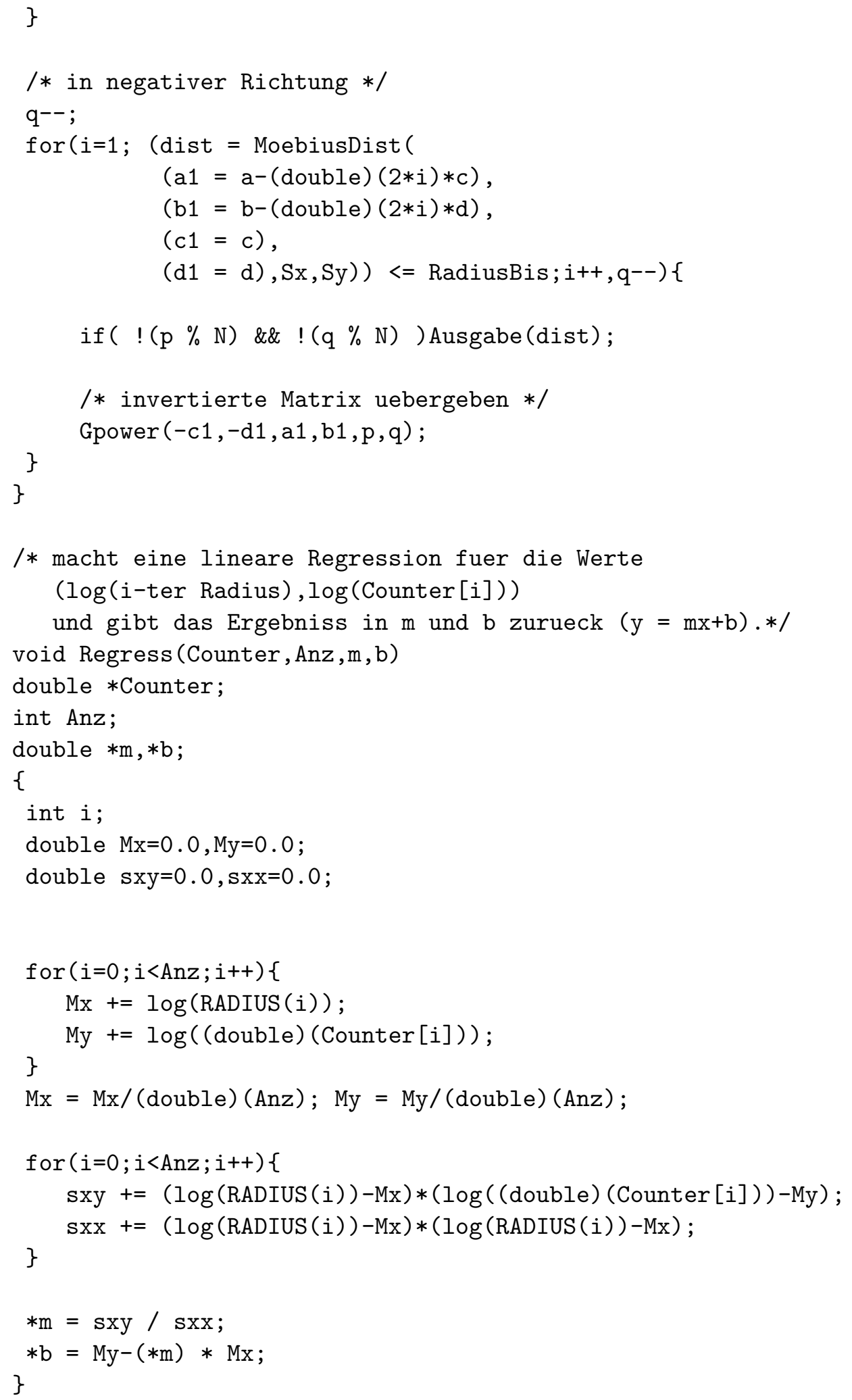




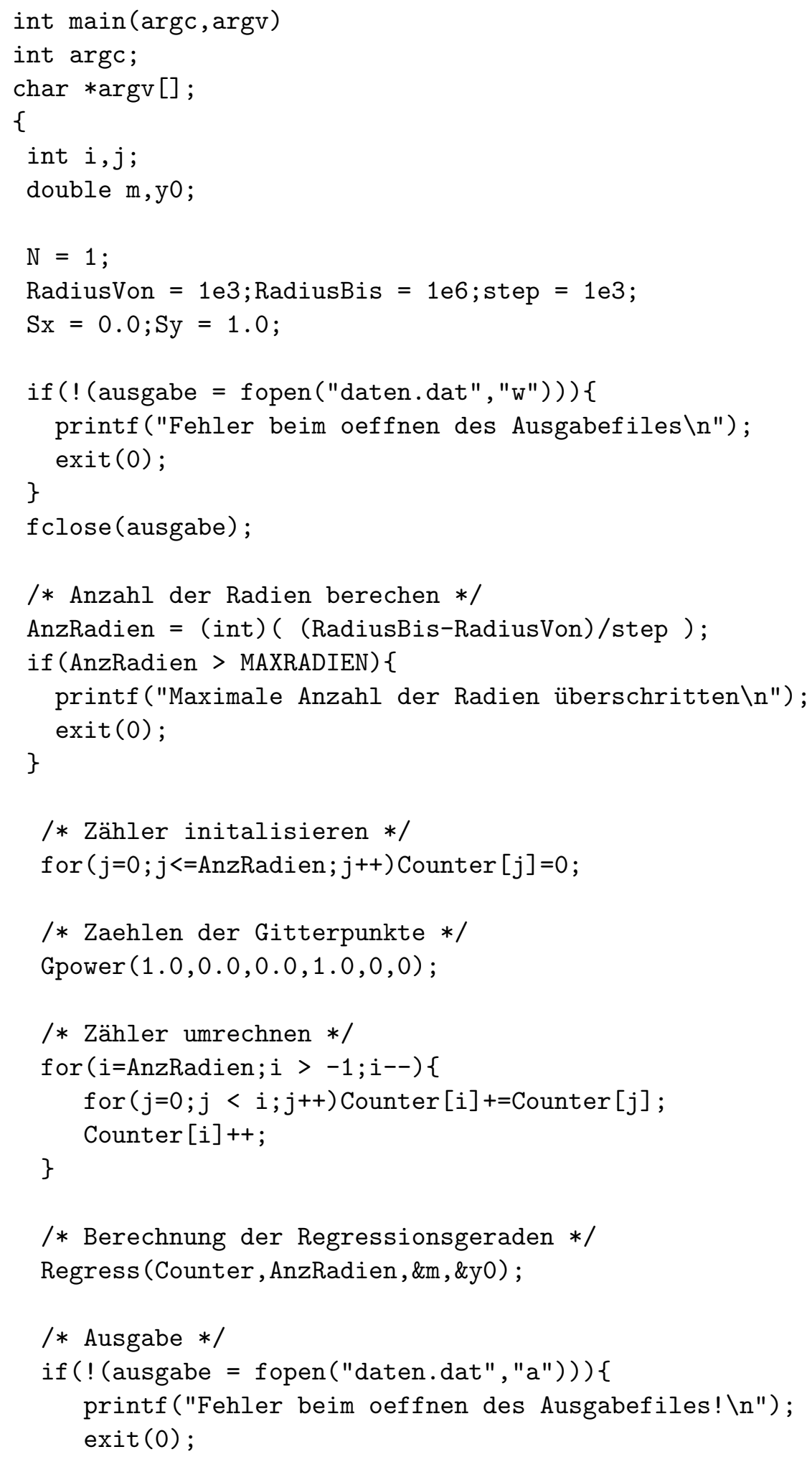




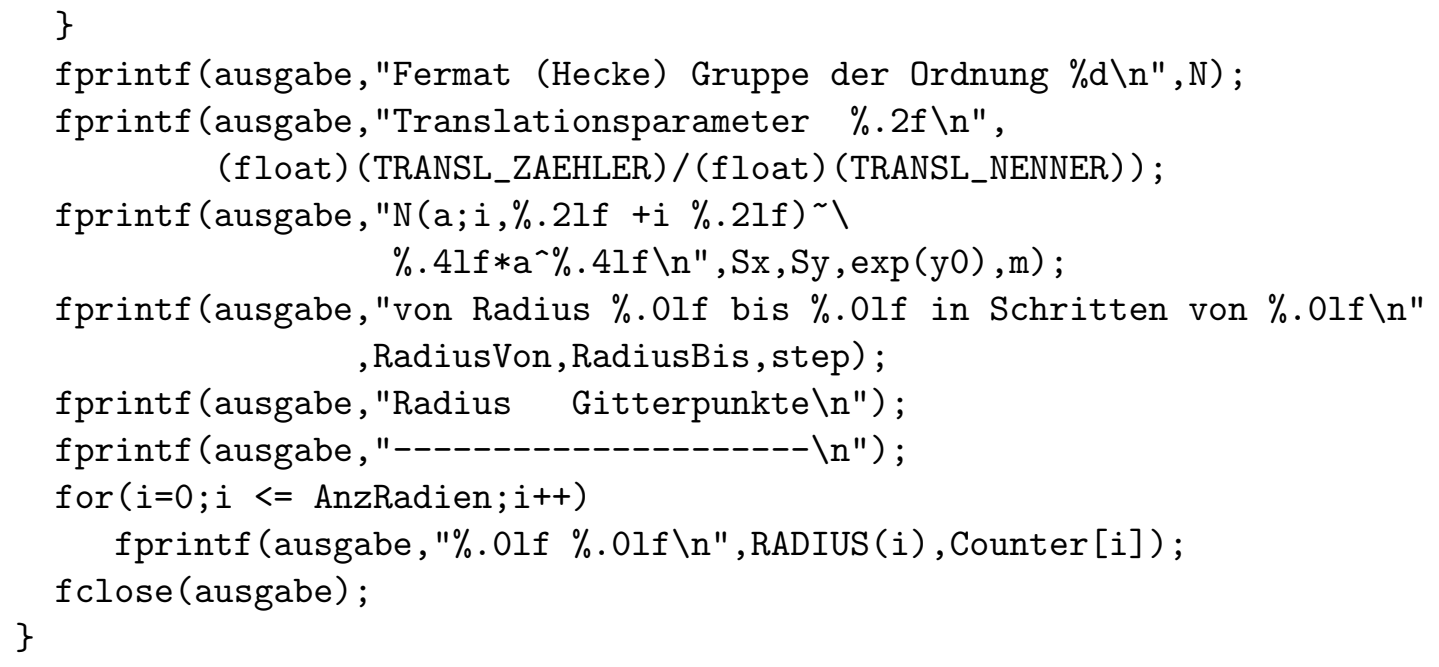

Um Heckegruppen auszählen zu können, muß das Programm nur wenig verändert werden. Wir konjugierten die Heckegruppen noch mit $z \mapsto 2 z$, um mit dem Programm vom Punkt $2 i$ (der anders als $i$ kein Verzweigungspunkt ist) zählen zu können. Die konjugierte Heckegruppe wird von

$$
z \mapsto z+\mu / 2, \quad z \mapsto-\frac{1}{4 z}
$$

erzeugt. Nur die Funktionen Gpower und Hpower müssen ersetzt werden:

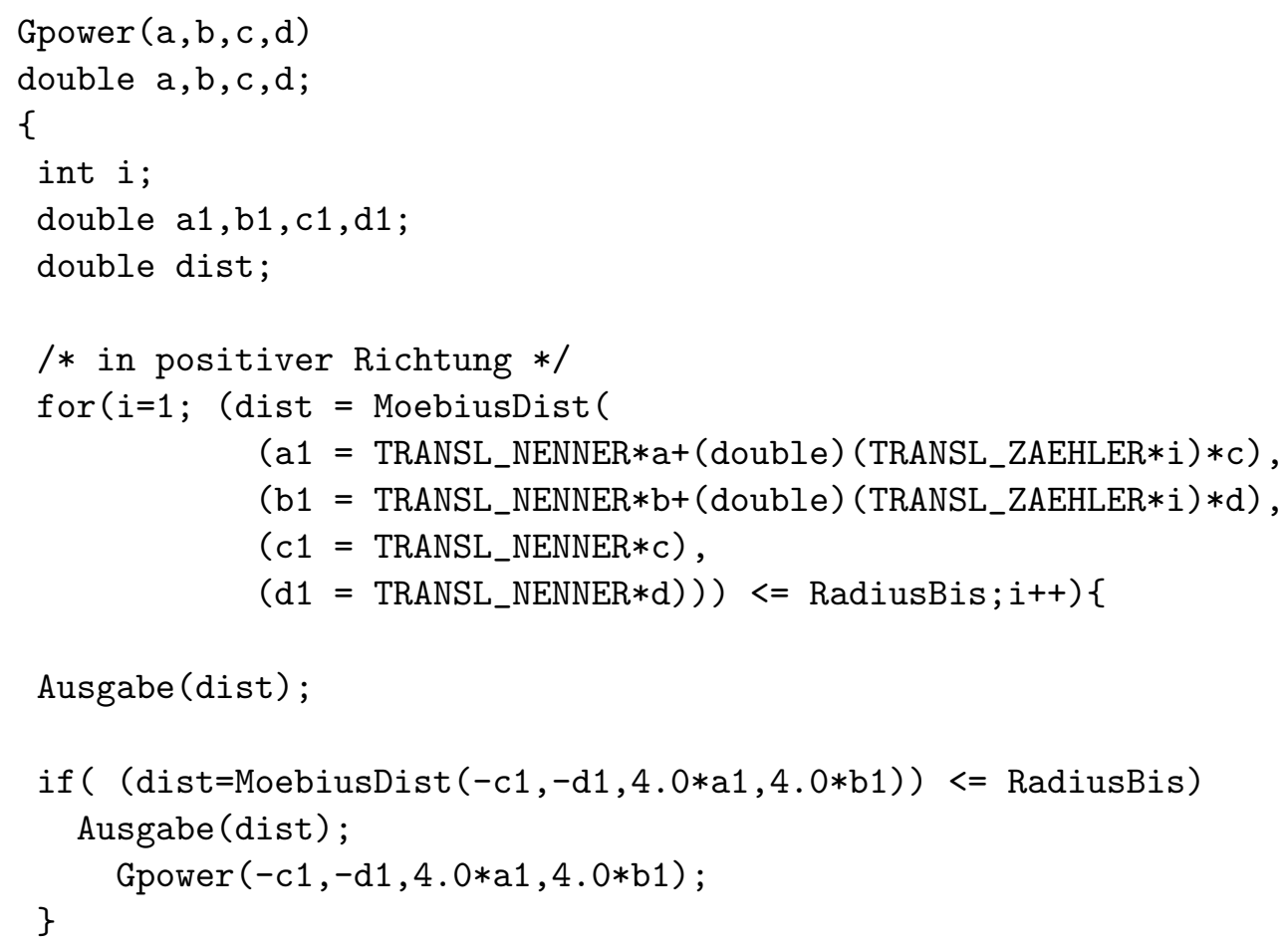




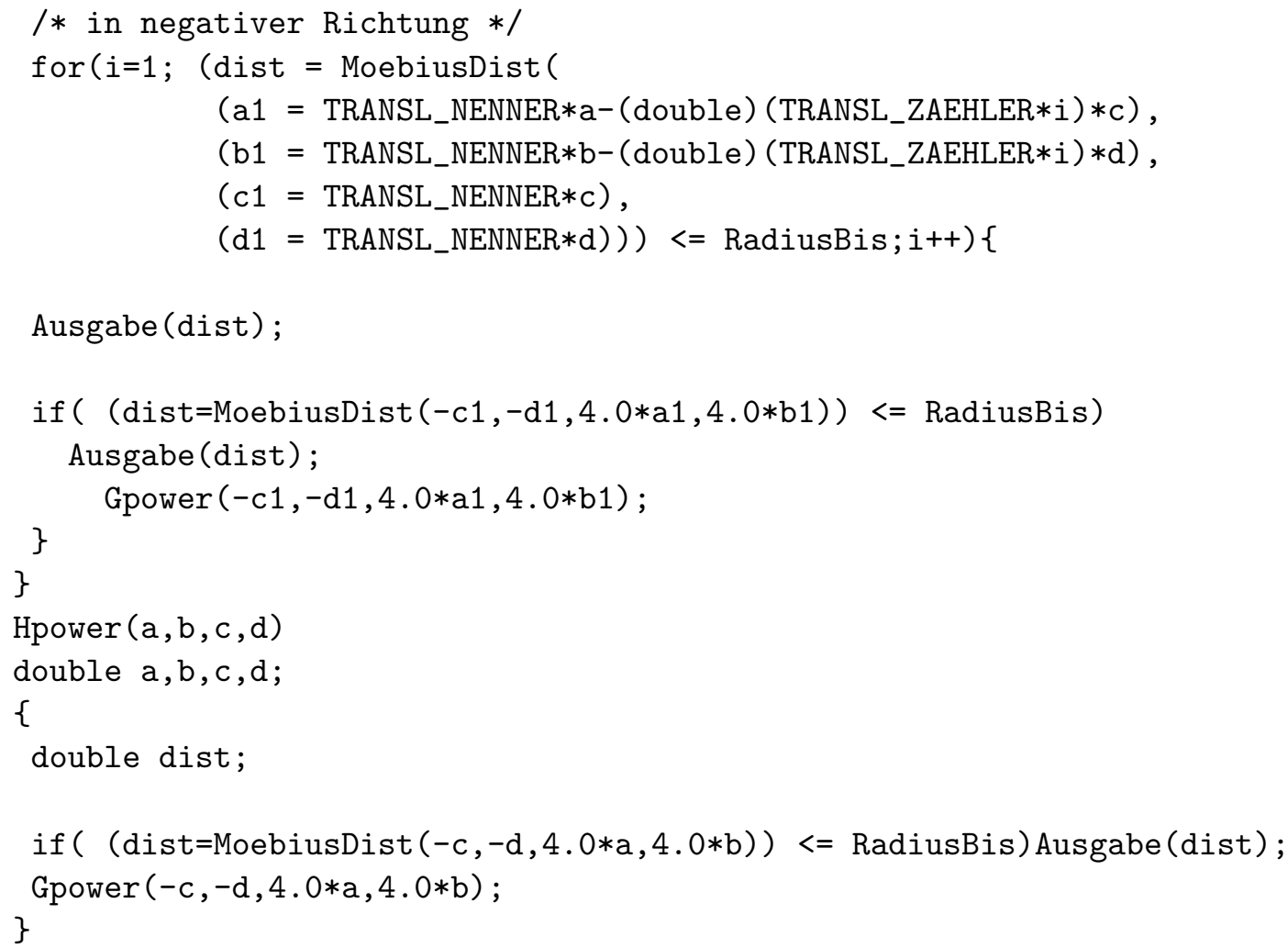

Die Gruppe $\Phi(N)$ mit $\mu=2$ hat eine Fundamentalbereich vom Flächeninhalt $2 \pi N^{2}$. Es gilt also

$$
N(a, z, w) \sim \frac{1}{N^{2}} \cdot a .
$$

Mit dem obigen Programm wurden für $N=1,7,8,9$ und $\mu=2$ die Werte von

$$
d(a ; i, i ; N):=N(a ; i, i ; N)-\frac{1}{N^{2}} \cdot a
$$

für $a=10^{7} \cdot k$ mit $k \in\{1, \ldots, 10000\}$ bestimmt. In der folgenden Abbildung 3.1 bis 3.4 sind die berechneten Funktionen $d(\cdot ; i, i ; N)$ mit $N=1,7,8,9$ dargestellt. Sie werden im Abschnitt 3.4 diskutiert. 


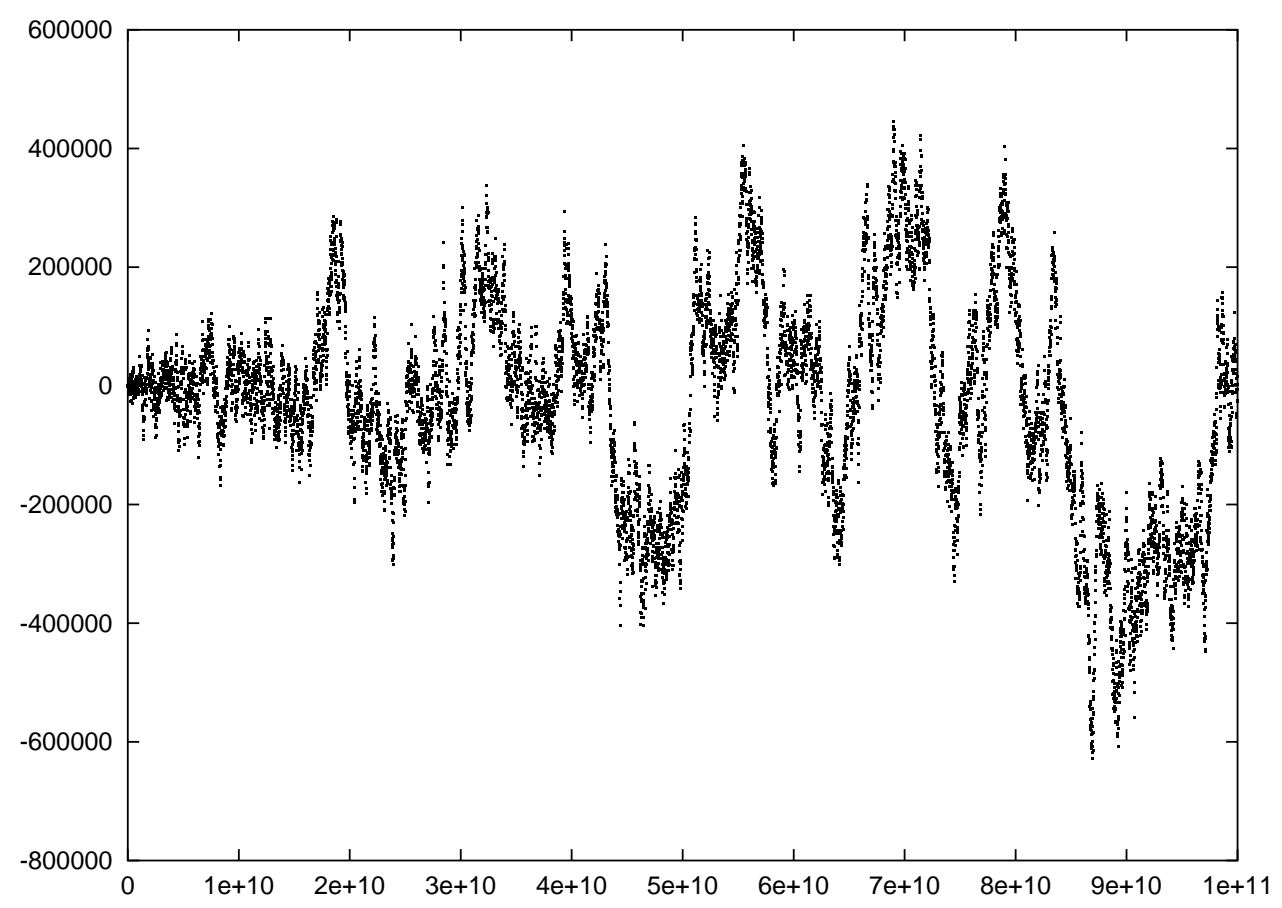

Abbildung 3.1.: Die Funktion $d(\cdot ; i, i ; 1)$

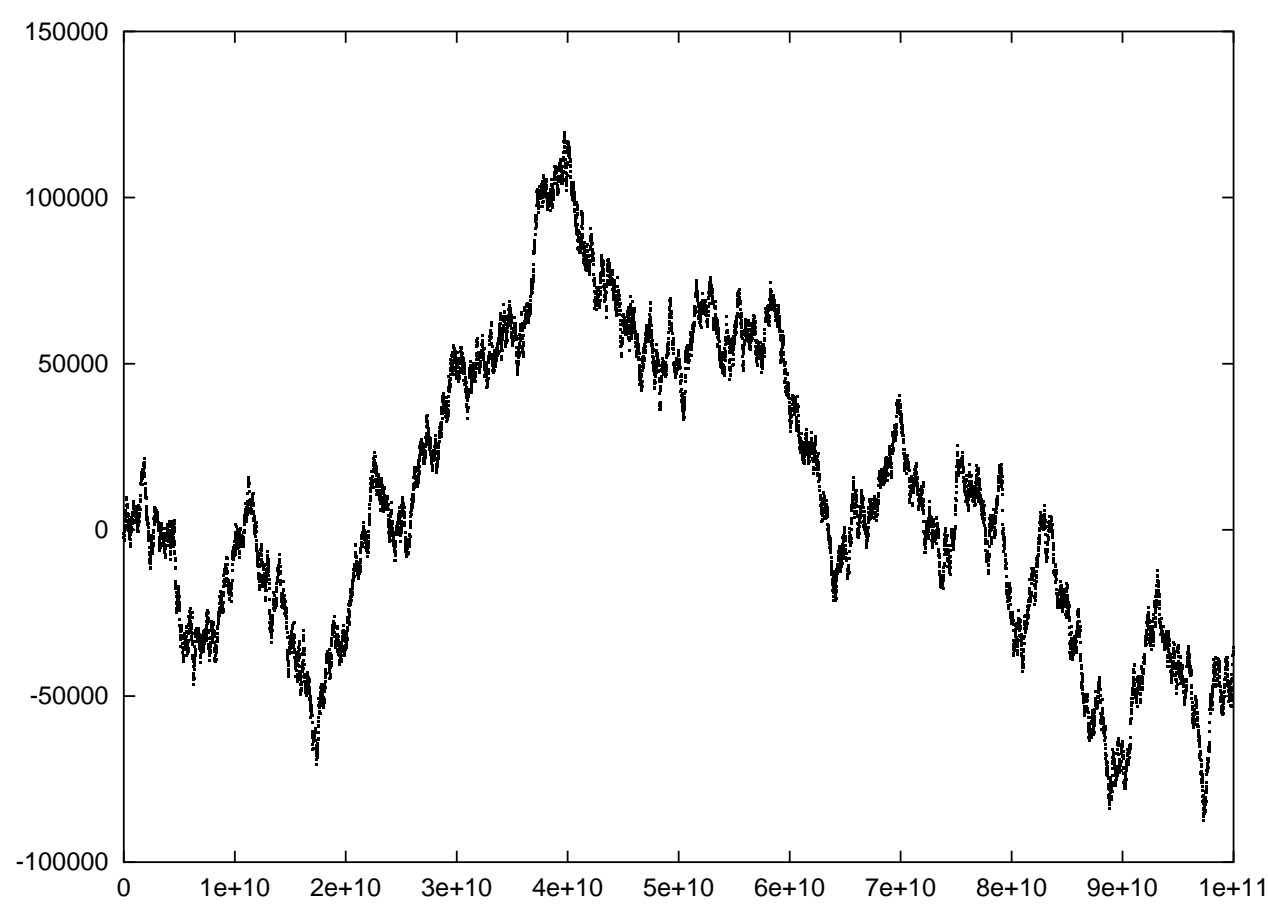

Abbildung 3.2.: Die Funktion $d(\cdot ; i, i ; 7)$ 


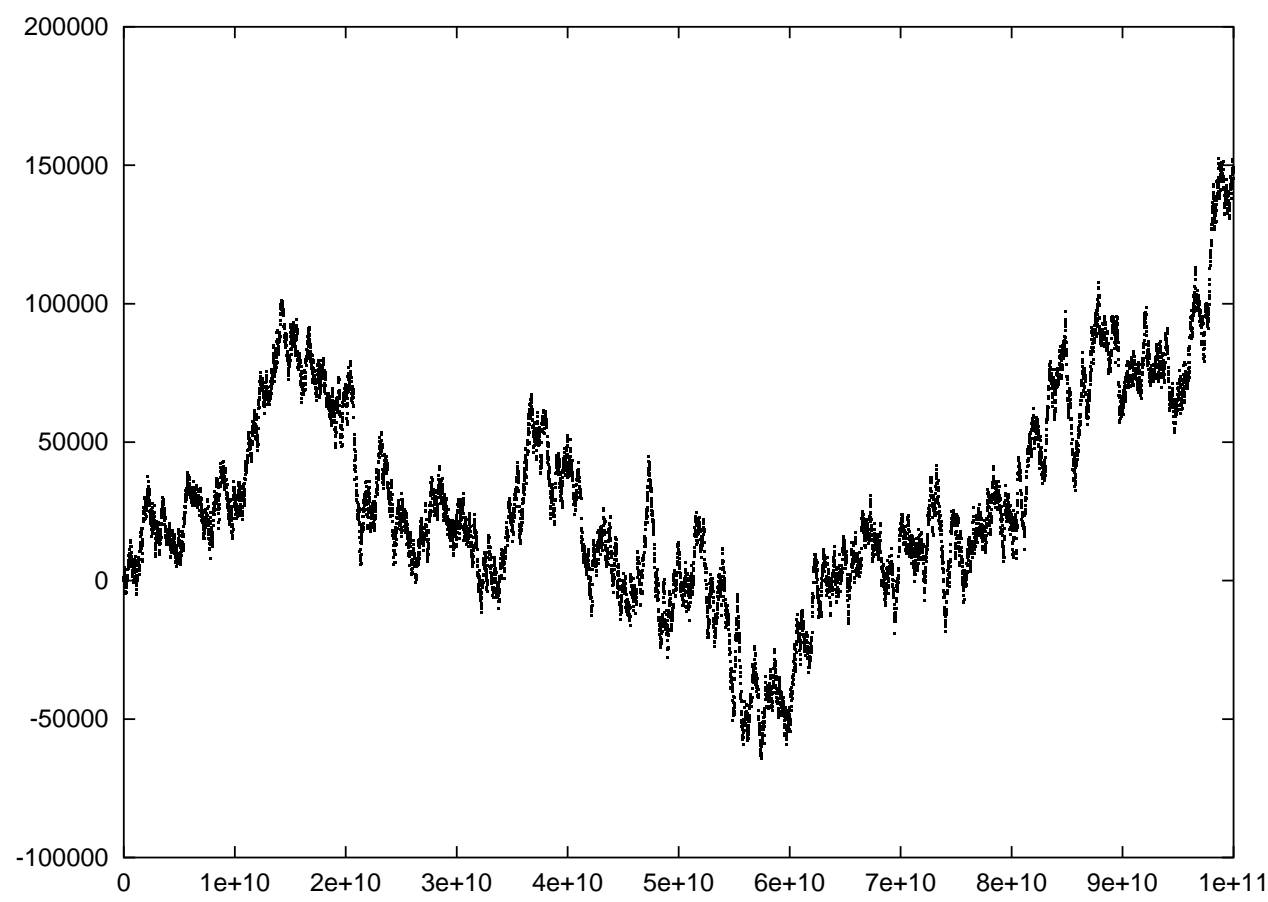

Abbildung 3.3.: Die Funktion $d(\cdot ; i, i ; 8)$

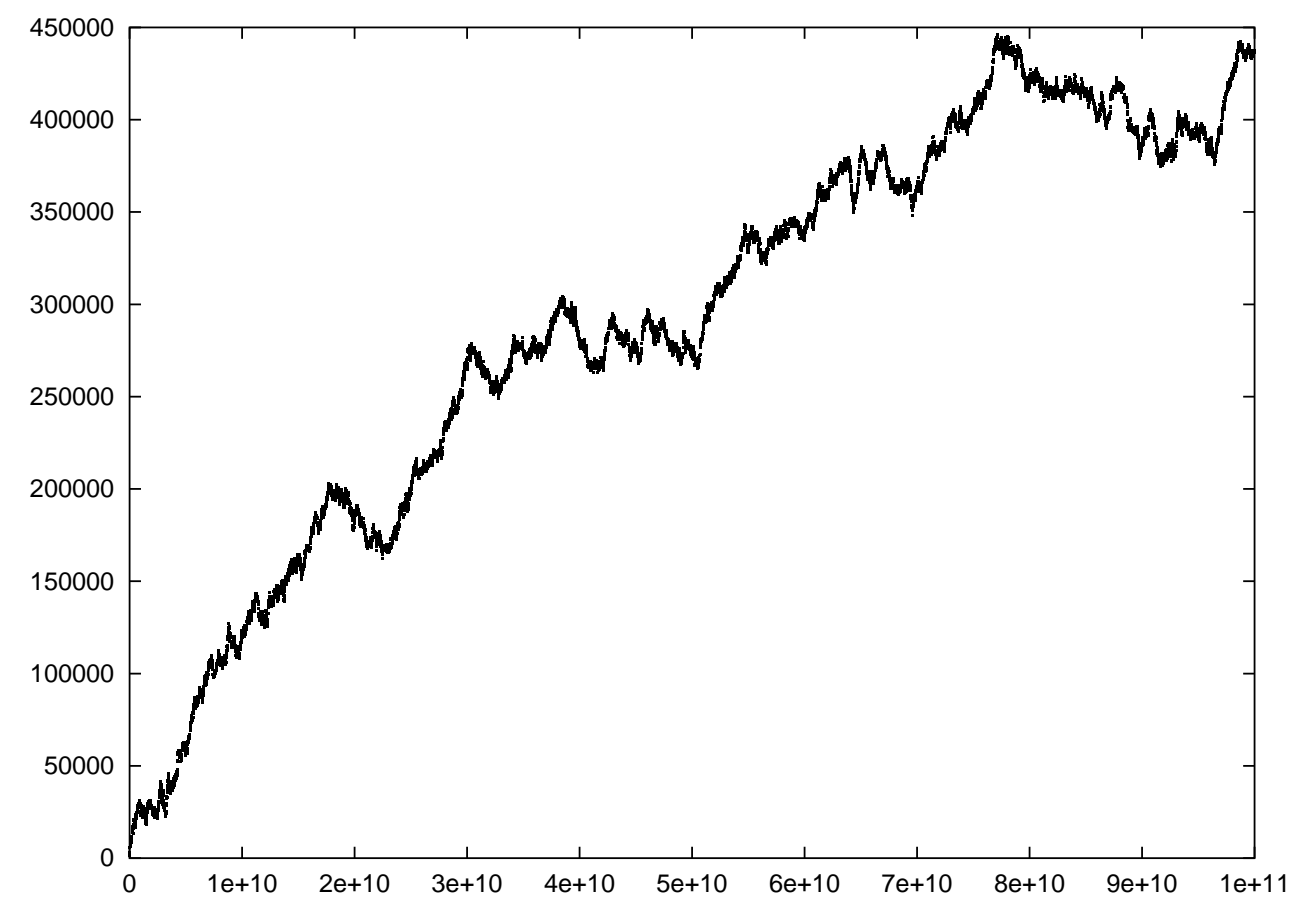

Abbildung 3.4.: Die Funktion $d(\cdot ; i, i ; 9)$ 


\section{Literaturverzeichnis}

[1] Beadron, A. F.: The geometry of discrete groups. Graduate Texts in Mathematics, 91. Springer-Verlag, 1983.

[2] Beardon, A. F.: The exponent of convergence of Poincaré series. Proc. London Math. Soc., 18(3):446-483, 1968.

[3] Buser, P.: Geometry and Spectra of Compact Riemann Surfaces. Birkhäuser, 1992.

[4] Corput, J. G. van DeR: Neue zahlentheoretische Abschätzungen. Math. Annalen, 89:215-254, 1923.

[5] Delsarte, J.: Sur le gitter fuchsien. C. R. Acad. Sci. Paris Sér I, 214:147-149, 1942.

[6] Delsarte, J.: Le gitter fuchsien. In: Ouevres de Jean Delsate, Band II, Seiten 829-845. Editions du Centre National de la Recherche Scientifique, Paris, 1971.

[7] Elstrodt, J.: Die Resolvente zum Eigenwertproblem der automorphen Formen in der hyperbolischen Ebene. I, Math. Ann. 203:293-330, 1973 ; II, Math. Z. 132:99-134, 1973 ; III, Math. Ann. 208:99-132, 1974.

[8] Günther, P.: Gitterpunktprobleme in symmetrischen Räumen von Rang 1. Math. Nachr., 94:5-27, 1980.

[9] HaRdy, G. H.: On the expression of a number as the sum of two squares. Quarterly Journal of Math., 46:263-283, 1915.

[10] Hobson, E. W.: The Theory of spherical and ellipsoidal harmonics. Cambridge At The University Press, 1931.

[11] Huber, H.: Zur analytischen Theorie hyperbolischer Raumformen und Bewegungsgruppen. Math. Ann., 138:1-26, 1959.

[12] HuBer, H.: Über den ersten Eigenwert des Laplace-Operators auf kompakten Riemannschen Flächen. Comment. Math. Helv., 49:251-259, 1974.

[13] Huber, H.: Über die Eigenwerte des Laplace-Operators auf kompakten Riemannschen Flächen. Comment. Math. Helv., 51:215-231, 1976.

[14] HuBer, H.: Über das Spektrum des Laplace-Operators auf kompakten Riemannschen Flälchen. Comment. Math. Helv., 58:627-647, 1982. 
[15] HubeR, H.: Über Gitter in der hyperbolischen Ebene. J. reine angew. Math., 458:127-156, 1995.

[16] HuXley, M.: Exponential sums and lattice points II. Proc. London Math. Soc., 66(2):279-301, 1993.

[17] Landau, E.: Über die Gitterpunkte in einem Kreis (II). Göttinger Nachrichten, Seiten 161-171, 1915.

[18] LandaU, E.: Vorlesung über Zahlentheorie II. Verlag von S. Herzel in Leipzig, 1927.

[19] LAX, P. D. und R. S. Phillips: The asymtotic distribution of lattice points in Euclidian and non-Euclidian spaces. J. Funct. Anal., 46:280-350, 1982.

[20] Littlewood, J. F. und A. Walfisz: The lattice points of a circle. Proc. R. Soc. Lond. A, 106:478-488, 1924.

[21] Maurin, K.: Methods in Hilbert spaces. Warszawa: PWN-Polish Scientific Publishers, 1967.

[22] Mazzeo, R. R. und Melrose, R. B.: Meromorphic extension of the resolvent on complete spaces with asymptotically constant negativ curvature. J. Funct. Anal., $75(2): 260-310,1987$.

[23] McMullen, C.: Hausdorff dimension and conformal dynamics III: Computation of dimension. Amer. J. Math., 120:691-721, 1998.

[24] Nicholls, P. J.: A lattice point problem in hyperbolic space. Michigan Math. J., 30:273-287, 1983.

[25] Nicholls, P. J.: The Erodic Theory of Discrete Groups, Band 143 der Reihe London Mathematical Society Lecture Note Series. Cambridge university press, 1989.

[26] Patterson, S. J.: A lattice-point problem in hyperbolic space. Mathematika, 22:8188, 1975.

[27] Patterson, S.J.: The exponent of convergence of Poincaré series. Monatsh. Math., 82:297-315, 1976.

[28] Patterson, S.J.: The limit set of a Fuchsian group. Acta Math., 136:241-273, 1976.

[29] Patterson, S.J.: Spectral theory and Fuchsian groups. Math. Proc. Camb. Philos. Soc., 81:59-75, 1977.

[30] Patterson, S. J.: Lectures on measures on limit sets of Kleinian groups. In: Analytical and geometric aspects of hyperbolic space, Band 111 der Reihe London Math. Soc. Lecture Note Ser., Seiten 281-323. Cambridge Univ. Press, CambridgeNew York, 1987. 
[31] Patterson, S.J.: On a lattice-point problem in hyperbolic space and related questions in spectral theory. Ark. Mat., 26, No.1:167-172, 1988.

[32] Phillips, R. und Z. Rudnick: The Circle Problem in the Hyperbolic Plane. J. Func. Analysis, 121:78-116, 1994.

[33] Phillips, R. und P. Sarnak: The Laplacian for domains in hyperbolic space and limit sets of Kleinian groups. Acta Math., 155:173-241, 1985.

[34] Phillips, R. und P. Sarnak: The spectrum of Fermat curves. Geom. Funct. Anal., 1:80-146, 1991.

[35] Roelcke, W.: Das Eigenwertproblem der automorphen Formen in der hyperbolischen Ebene. I, Math. Ann. 167:292-337, 1966 ; II, Math. Ann. 168:261-324, 1967.

[36] SelBerg, A.: Harmonic analysis and discontinuous groups in weakly symmetric Riemannian spaces with applications to Dirichlet series. J. Indian Math. Soc., n. Ser., 20:47-87, 1956.

[37] Selberg, A.: On discontinuous groups in higher-dimensional symmetric spaces. Contrib. Function Theory, Int. Colloqu. Bombay, Jan. 1960, Seiten 147-164, 1960.

[38] Selberg, A.: Harmonic analysis (Göttinger lectures). In: Collected Papers, Band I. Springer-Verlag, 1989.

[39] SiERPINSKI: O pewnem zagadnieniu z rachunku funkcyj asymptotycznych. Prace mathematyczno-fizyczne, 17:77-118, 1906.

[40] Stratmann, B. und Velani S. L.: The patterson measure for geometrically finite groups with parabolic elements new and old. Proc. London Math. Soc., 71(3):197220, 1995 .

[41] Watson, G. N.: Theory of Bessel Functions. Cambridge at the university press, 1922 . 


\title{
Lebenslauf
}

\author{
Name Jan Thirase \\ Geburtsdatum 12. Februar 1971 \\ Geburtsort Lüneburg \\ Schulbildung 1977 - 1981 Grundschule der Freiherr vom Stein \\ Schule, Eppstein \\ 1981 - 1987 Gymnasialzweig der Freiherr vom Stein \\ Schule, Eppstein \\ 1987 - 1990 Oberstufe an der Main Taunus Schule, Hofheim \\ 20.6.1990 Abitur \\ Studium 1990-1993 Grundstudium der Mathematik und Physik \\ 1992 Vordiplom in Physik \\ 1993 Vordiplom in Mathematik \\ 1997 Diplomprüfung Mathematik und Beginn des \\ Promotionsstudienganges Mathematik \\ wiss. Tätigkeit 1997-1998 Wissenschaftlicher Mitarbeiter am \\ Mathematischen Institut der Universität Göttingen \\ 1998-2000 Stipendium der Graduiertenförderung \\ des Landes Niedersachsen \\ seit April 2000 Wissenschaftlicher Mitarbeiter am \\ Mathematischen Institut der Universität \\ Göttingen, finanziert durch die DFG
}

